\title{
ASYMMETRIC QUANTUM WELL STRUCTURES FOR ENHANCED INFRARED PHOTON ABSORPTION
}

\author{
A Dissertation Presented to \\ The Faculty of the Graduate School \\ at \\ The University of Missouri,Columbia \\ in Partial Fulfillment of the Requirement \\ for the Degree of \\ Doctor of Philosophy
}

by

MEHJABEEN A. KHAN

Professor Naz Islam, Dissertation Supervisor

JULY 2013 
The undersigned, appointed by the dean of the Graduate School, have examined the dissertation entitled

\section{ASYMMETRIC QUANTUM WELL STRUCTURES FOR ENHANCED INFRARED PHOTON ABSORPTION}

presented by Mehjabeen A Khan, a candidate for the degree of doctor of philosophy

and hereby certify that, in their opinion, it is worthy of acceptance.

Professor Naz Islam

Dr. Mahmoud Almasri

Dr. Guilherme N DeSouza

Professor H. R. Chandrasekhar 


\section{ACKNOWLEDGEMENT}

I would like to take the opportunity to acknowledge the contributions from my caring surrounding people at the end of one of the most important phases of my life at MU. First and foremost I would like to thank Professor Naz Islam for his extraordinary support and advising in all steps of my dissertation. His inspiring guidance has been one of the strongest driving forces behind my research work.

I am very grateful to the members of my thesis committee: Dr. Mahmoud Almasri, Dr. G. N. DeSouza and Dr. Chandrasekhar, for their remarks and thorough consideration of the document. I can never thank enough my husband Dr. Pavel, who's in depth knowledge in solid state device physics helped me solving many of my problems. I would also like to thank the Department of Electrical and Computer Engineering for supporting me through teaching assistantship. I am especially indebted to Dr. Almasri for allowing me to use his laboratory resources. In this connection, I would like to thank members of his research group specifically M. Lutful Hai for their outstanding support.

I thank my parents who have blessed my life with their support, inspiration and unconditional love. Their presence and care to me and my daughter made my journey smooth towards the end of crucial last year of my dissertation. Next, I would like to express my in-debt gratitude to my wonderful friends at the University of MissouriColumbia, each of whom has been a source of inspiration in their own way. 
Last but not the least; I would like to thank my beloved husband and wonderful daughter, Renata Akeed, the mere presence of whom in my life has made it worth living. Their support and love have made my life full by bringing the greatest happiness I have ever known. 


\section{TABLE OF CONTENTS}

Acknowlwdgement

List of Figures

vii

Abstract

Chapter 1

$\begin{array}{ll}\text { Introduction } & 1\end{array}$

$\begin{array}{lll}\text { An Overview of Absorption Mechanism } & 3\end{array}$

$\begin{array}{lll}1.2 & \text { Asymmetric Quantum-well structure } & 8\end{array}$

$\begin{array}{lll}1.3 & \text { Thesis Outline } & 12\end{array}$

\section{Chapter 2}

Optical Absorption in Quantum Wells

2.1 Infrared Photodetection

2.2 Traditional interband based detectors and intraband QWIPs

2.3 Quantum well Infrared Photodetector (QWIP)

2.4 Intersubband absorption between bound states

2.5 Asymmetric Quantum Well

2.6 Dark current 
2.7 Structure and Material Consideration

2.8 Conclusion

Chapter 3

Quantifying Inter-subband transition in Asymmetric Quantum Wells

3.1 Quantifying Absorption Rate 53

3.2 Impact of Effective mass and Strain 56

3.3 Selection Rule and Normal Incidence 58

3.4 NEGF and Quantum wave impedance 68

3.5 Conclusion 76

\section{Chapter 4}

Analysis of Asymmetric Quantum Wells for Infrared

\section{Photodetection}

4.1 Analysis of AlGaAs/GaAs/InGaAs n-type step quantum wells

4.2 Effect of Asymmetry on Overall Absorption

4.3 Simulation Setup for a Stepped Quantum Well

4.4 Effect of Step Width on Absorption

4.5 Impact of Strain

4.6 Multiple Wavelength Detection and Voltage Tunability

4.7 Conclusion 


\section{Chapter 5}

Summary and Concluding Remarks

5.1 Asymmetric Quantum Wells and Material Choice 106

$\begin{array}{lll}5.2 & \text { Quantifying Optical absorption and Carrier Transport } & 107\end{array}$

5.3 Analyzing the Symmetry Breaking Through Asymmetric 108 QW

5.4 Future Work

110

$\begin{array}{lr}\text { References } & 112\end{array}$

$\begin{array}{ll}\text { Vita } & 114\end{array}$ 


\section{LIST OF FIGURES}

1.1 (a) Intrinsic Photodetector,

(b) Extrinsic Photodetector

1.2 Band structure of Quantum-well inter-sub-band absorption between electron levels $E_{1}$ and $E_{2}$ or levels $H_{1}$ and $H_{2}$ is schematically shown

1.3 Bound-to-bound inter-sub-band transition followed by tunneling 6 out of the well

1.4 A GaAs/AIGaAs QWIP structure which employs bound-tocontinuum

1.5 AlGaAs $/ \operatorname{In}_{\mathrm{x}} \mathrm{Ga}_{(1-\mathrm{x})} \mathrm{As} / \mathrm{In}_{\mathrm{y}} \mathrm{Ga}_{(1-\mathrm{y})} \mathrm{As} / \mathrm{AlGaAs} \mathrm{QWIP}$ structure

1.6 Energy band diagram of a double quantum well structure for voltage tunable photodetector: (a) in equilibrium, (b) positive voltage applied and (c) negative voltage applied

2.1 Spectral radiance $L_{B B}$ of blackbodies at different temperatures $T$

2.2 Schematic of a photodetection mechanism, showing a phodetector biased and connected to external detection circuitry

2.3 Schematics of conduction band profile of a quantum well structure absorbing radiation

2.4 Band structure of quantum-well (depths $\Delta E_{c}$ and $\Delta E_{v}$. 26 Intersubband absorption between electron levels $E_{1}$ and $E_{2}$ or hole levels $H_{1}$ to $H_{2}$ is schematically shown

2.5 Measured bound-to-bound QWIP intersubband absorbance=-log (transmission) vs photon energy at Brewster's angle $\theta_{B}=7^{\circ}$

2.6 Measured QWIP absorbance vs photon energy for the multipass waveguide geometry shown in the insert

2.7 Measured QWIP absorbance vs angle of incidence $\theta$ (dots), and theoretical dependence (solid curve) 
2.9 Stepped quantum well indicating that the $E_{1}$ to $E_{2}$ transition and

2.10 Dark current transport in a typical QWIP. The three mechanisms shown are sequential resonant tunneling (ISRT), thermionic emission (ITE) and thermally assisted tunneling (ITAT). At low bias, $\phi_{b}=\Delta E_{c}-E_{0}-E_{F}$ is the barrier height seen by electrons in the ground state $E_{0}$ of the QWs. $\Delta E_{c}$ is the conduction band offset and $E_{F}$ the Fermi energy measured from $E_{0}$

2.11 (a) Sequential resonant tunneling in high-field domain (right-hand side); tunneling through ground state (left-hand side).

(b) Photoconductivity produced by absorption of intersubband radiation followed by tunneling out of well

2.12 Schematic band diagram of the superlattice for several values of the average potential drop per period $V_{P}$ (a) Zero bias. (b) Sequential resonant tunneling through the ground state $\boldsymbol{E}_{1}$ for $V_{p}<2 \hbar / \tau_{1}$; arrows indicate electron transport. (c) Formation of first high-field domain for $V_{P}$ slightly greater than $2 \hbar / \tau_{1}$. Sequential resonant tunneling occurs through $E_{1}$ in the low-field region and through the first excited state $E_{2}$ in the high-field region. (d) Expansion of the high-field domain by one additional quantum well for a voltage increase of $\Delta V$

2.13 QWIP structure which employs bound-to-continuum

2.14 Conduction-band structure of a biased three terminal QWIP(emitter $\mathbf{E}$, base B, and collector $\mathrm{C}$ ). The collector barrier blocks the tunneling current from the ground state $E_{1}$

3.1 Band structure of GaAs. $300 \mathrm{~K}$

3.2 Schematic representation of a perfect square $Q W$ and the wavefunctions of its bound states. The figures show the inversion symmetry of the wavefunctions

3.3 A step potential barrier illustrating the concept of quantum wave impedance 
3.4 Representation of DOS as a function of energy that reveals the broadening of energy states and FWHM, along with carrier lifetime on a given energy state

$\begin{array}{llllllll}\text { 4.1 } & \text { Schematic } & \text { representation of } & \text { a } & \text { single } & \text { period } & \text { of } & 79\end{array}$ AlGaAs/GaAs/InGaAs quantum well structure

4.2 Schematic representation of asymmetric/stepped quantum well showing step width and height

4.3 Conduction band profile and wavefunctions for a

$\mathrm{Al}_{x} \mathrm{Ga}_{1-x} \mathrm{As} / \quad \mathrm{In}_{y} \mathrm{Ga}_{1-y} \mathrm{As} / \mathrm{GaAs}$ with $15 \%$ Al and $15 \%$ In concentration. The wavefunctions associated with the ground state and first excited states are shown in blue and purple dotted lines

4.4 Calculated Density of states (DOS) and absorption coefficient for the structure shown in Figure: 4.3

4.5 Calculated magnitudes of wavefunctions (with their energies shown 86 on the vertical axis) for $\mathrm{Al}_{0.15} \mathbf{G a}_{0.85} \mathrm{As} / \mathrm{In}_{0.15} \mathrm{Ga}_{0.85} \mathrm{As} / \mathrm{GaAs}$ structure. The blue dotted lines are for rectangular QW $\left(\mathrm{Al}_{0.15} \mathrm{Ga}_{0.85} \mathrm{As} / \mathrm{GaAs}\right)$

4.6 $\alpha_{i f}$ for a 30nm-6nm-6nm $\mathrm{Al}_{x} \mathrm{Ga}_{1-x} \mathrm{As} / \mathrm{GaAs} / \mathrm{In}_{y} \mathrm{Ga}_{1-y} \mathrm{As}$ stepped $\mathrm{QW}$ and it's square $Q W$ counterpart. Inset shows the optical broadening for different transitions

4.7 $\Gamma$ $\Gamma$ op $/ \Gamma_{t u n}$ and $\alpha$ calculated as a function of Indium concentration

4.8 Conduction band profile of $\mathrm{AlGaAs} / \mathrm{In}_{\mathrm{x}} \mathrm{Ga}_{(1-\mathrm{x})} \mathrm{As} / \mathrm{GaAs} / \mathrm{AlGaAs}$ structure

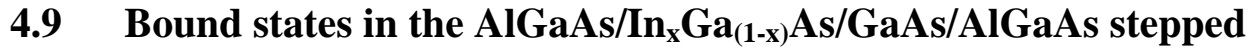
quantum well

4.10 Electron wavefunctions of the bound states

4.11 Absorption in the structure

4.12 Spectral response of the structure

4.13 Effect of strain on conduction band profile and wavefunctions 
4.15 Multiple quantum well structures demonstrating multiple wavelength detection. Top figure is at zero bias, whereas the bottom one is biased at $1 \mathrm{~V}$

4.16 Increased wavelength separation achieved through increased step- 102 width and reversed applied voltage

5.1 Modified potential profile for dark current reduction 


\section{$\underline{\text { Abstract }}$}

Compared to inter-band transition for photon absorption in a quantum wells, intra-band (or inter-subband) transitions in heterojunction (GaAs/InP) quantum wells can provide access to a broader range of wavelengths for detector design, specifically detectors operating in the mid infrared region of spectrum $(4-12 \mu \mathrm{m})$ and beyond is possible. These quantum wells not only provide great flexibility in optimizing the Eigen energy levels or wavefunctions, and inter-subband optical matrix elements determining the corresponding transition probability, but also allow controlling electron-phonon scattering rates and thus electron lifetime.

The research presented in this dissertation investigates asymmetric quantum well structures formed through III-V semiconductor material system such as $\mathrm{AlGaAs} / \mathrm{In}_{\mathrm{x}} \mathrm{Ga}_{(1-}$ x) $\mathrm{As} / \mathrm{In}_{\mathrm{y}} \mathrm{Ga}_{(1-\mathrm{y})} \mathrm{As} / \mathrm{AlGaAs}$ that can further improve the responsivity through higher carrier mobility. Asymmetry is introduced by using multiple materials to form the well region. The advantage of exploring stepped quantum well structure stems from experimental evidence that such structures are capable of absorbing normal incidence and thus eliminates the requirement of incorporating additional optical coupling schemes such as grating structures. An important contribution of this research is the development of an analytical model to analyze single or multiple quantum well structures to quantify photon absorption. The physical model developed in this work is based on non-equilibrium Green's function (NEGF), Fermi's golden rule and quantum mechanical wave impedance concept. The approach has two distinct advantages. First, it is accurate, easily programmable and yet computationally efficient. Second, it facilitates quantifying the 
broadening of states resulting from both photon absorption and tunneling, which provides important insight for improving detection efficiency. Instead of being presented through calculations, such broadening has been simply assumed in previously reported works. The method developed in this research provides an efficient methodology to quantify the absorption rate of photons with different polarizations.

The study looks into the design and analysis of the wells to enhance infrared radiation detection, and suggests mechanisms to optimize the structures in terms of material choice, composition, structural geometry and applied voltages. The high electron mobility and hence drift velocity in InGaAs $(>5 \times 106 \mathrm{~cm} / \mathrm{s})$, along with low carrier recapture lifetime, is expected to improve the detection speed of the device. Unlike GaAs/AlGaAs, the lattice mismatch at InGaAs/AlGaAs interface is likely to introduce strain in the structure. The effect of such strain as well as the layer thickness is taken into account in this work. Also, a methodology for achieving voltage tenability, i.e. the ability to detect different wavelengths at different bias voltages has been investigated.

Although both $n$-type and $p$-type systems have potential for optoelectronic devices, $n$ type quantum wells are more advantageous because of their nearly parabolic sub-band structure. As for material consideration, direct band gap material based systems, such as AlGaAs/InGaAs and AlGaAs/GaAs, have been found to be particularly suitable for $n$ type QWIP photodetectors. The reason being that the electrons in such materials occupy states around the $\Gamma$ valley where the effective mass is smaller and therefore mobility is higher; a condition which leads to higher photoresponsivity. Moreover such systems allow the possibility of highly stable device operation owing to the insensitivity of intra- 
band transition energies to temperature fluctuation and reduced influence of Auger recombination. 


\section{CHAPTER 1}

\section{Introduction}

Infrared (IR) photodetectors sensitive to $2-10 \mu \mathrm{m}$ radiation play vital roles in thermal imaging and sensing, which has important commercial and defense applications. Understandably, mechanisms and material considerations and characteristics that may lead to improved photondetection in the infrared regime have been rigorously investigated. Among the devices that are being explored, quantum well structures have been shown to be the most versatile for infrared detection, as by merely changing the material composition, one can tune the detection wavelength anywhere from 2 to $35 \mu \mathrm{m}$ and beyond [1]. The quantum well photodetectors are fabricated using Molecular Beam Epitaxy (MBE), which is a well matured materials growth and process technology capable of producing ultra-pure and thin films on larger substrates [2].

A quantum well IR photodetector (QWIP) absorbs photons that involve transitions among sub-bands in quantum wells which connect electronic states within the same band (conduction or valence band). Compared to inter-band transition, intra-band (or inter-subband) transition provides access to much lower energy, allowing device operation at longer wave lengths. The intra-band transitions were first investigated during 1960s in the context of two-dimensional electron gas confined within the quantum well at silicon/silicon dioxide interface [3]. The intra-band transition is particularly suitable for 
photodetectors as they ensure covering of a broad range of wavelengths using the combinations of GaAs and InP based alloys that are used for state-of-the-art electronics and optical communications. Therefore the major impetus for engineering new photonic materials and optoelectronic devices operating in the mid infrared region of spectrum (4$12 \mu \mathrm{m})$ and beyond came from the subsequent investigation on GaAs/InP based heterojunction quantum wells [3]. These heterojunction quantum wells not only provide greater flexibility in optimizing the eigen energy levels or wavefunctions, and intersubband optical matrix elements determining the corresponding transition probability, but also electron-phonon scattering rates and thus electron lifetime.

Although both $n$-type and $p$-type systems have potential for optoelectronic devices, $n$-type quantum wells are more advantageous because of their nearly parabolic sub-band structure [4]. As for material consideration, direct band gap material based systems, such as $\mathrm{AlGaAs} / \mathrm{InGaAs}$ and $\mathrm{AlGaAs} / \mathrm{GaAs}$, have been found to be particularly suitable for $n$-type QWIP photodetectors. The reason being that the electrons in such materials occupy states around the $\Gamma$ valley where the effective mass is smaller and therefore mobility is higher; a condition which leads to higher photoresponsivity [5]. Moreover such systems allow the possibility of highly stable device operation owing to the insensitivity of intra-band transition energies to temperature fluctuation and reduced influence of Auger recombination [4].

This thesis presents an investigation on QW structures, composed of high electron mobility material like InGaAs, that has certain asymmetry introduced through either stepped well formed by of different materials (can be varied in terms of composition) or spatial variation of doping in the well region. Use of stepped quantum wells, for 
example, formed through $\mathrm{AlGaAs} / \mathrm{In}_{\mathrm{x}} \mathrm{Ga}_{(1-\mathrm{x})} \mathrm{As} / \mathrm{In}_{\mathrm{y}} \mathrm{Ga}_{(1-\mathrm{y})} \mathrm{As} / \mathrm{AlGaAs}$ structure can also improve the wavelength tunability of the QWIP. The high electron mobility and hence drift velocity in $\operatorname{InGaAs}(>5 \times 106 \mathrm{~cm} / \mathrm{s})$, along with low carrier recapture lifetime, is expected to improve the detection speed of the device. However one of the main motivations behind exploring stepped quantum well structure stems from the experimental evidences that such structures are capable of absorbing normal incidence and thus would eliminate the requirement of incorporating additional optical coupling schemes such as grating structures. Unlike GaAs/AlGaAs, the lattice mismatch at InGaAs/AlGaAs interface is likely to introduce strain in the structure, which the work presented in this thesis takes into account and minimizes by optimizing the thickness of different layers. The methodology of achieving voltage tenability, i.e. the ability to detect different wavelength at different bias voltages has also been demonstrated. The next section (1.1) provides an overview of photon absorption physics outlining different photodetection techniques with their limitations and advantages. This would be followed by the section 1.2 that briefly describes the asymmetric QW structures form through $\mathrm{AlGaAs} / \mathrm{InGaAs}$ stepped quantum wells that has been explored in this research. The concluding section of this chapter (1.3) would outline the next chapters that describe the detailed physics concepts, model development, calculation and simulation approach, and results of this work.

\subsection{An Overview of Absorption Mechanism}

Unlike detection of objects using visible light that requires a light source, infrared detectors can detect objects by detecting their natural heat emission. It is this non- 
reflective detection mechanism that makes infrared photodetectors particularly useful for certain applications, such as night vision. Semiconductor photodetectors absorb photons and generate charged carriers (electrons and holes), which are collected in the external circuit as photocurrent. The detectors that absorb photons of wavelength close to its band gap that excite band to band transition of carriers are called intrinsic photodetectors and are shown in Figure:1.1(a). In an extrinsic photodetectors, on the other hand, the absorption of a photon involves deep impurity or defect levels within the band gap. The extrinsic photodetection mechanism is shown in Figure: 1.1(b).

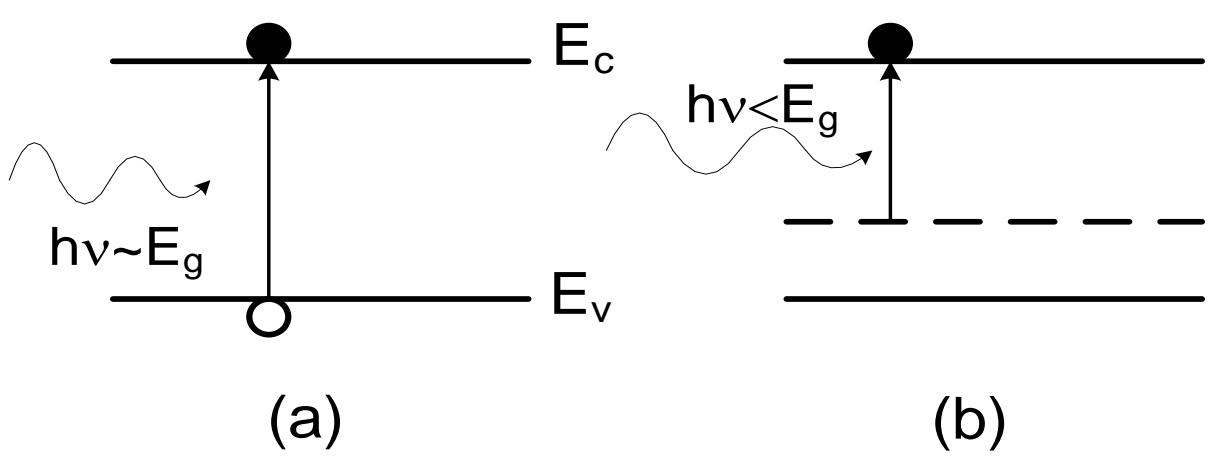

Figure: 1.1 (a) Intrinsic Photodetector, (b) Extrinsic Photodetector

These detectors, therefore, can detect photons with energy smaller than the band gap. The most commonly used infrared detectors include those that use mercury $(\mathrm{Hg})$, tellurium (Te) and cadmium (Cd) and therefore are known as $M C T$ detectors. Although MCT detectors are capable of operating at moderate temperatures $(\sim 70 \mathrm{~K})$, the main technological problem that has reduced their popularity is associated with weak $\mathrm{Hg}-\mathrm{Te}$ and Cd-Te bond, which results in bulk, surface and interface instabilities. Since in these devices photon detection is accomplished by exciting valence electron to the conduction band, preferably in the depletion region of a $p-n$ junction, and subsequently collecting the 
photo-generated carriers, the sensitivity is heavily influenced by the structural quality of the film [6-8]. Due to the poor bonds in MCT detector, trap density becomes inevitably high, which results in low carrier lifetime. This makes MCT structures not very suitable for fabricating large area imaging devices.

A variation of extrinsic photodetector, termed as intersubband photodetectors and has been the focus of this work, involves transitions of charged carriers between subband energies in a quantum well. QWIPs are based on such intersubband optical transition within a single energy band. In case of IR detection, the amount of energy transition is very small and this motivates the study of transitions between energy levels within the same band in a quantum well. These discrete energy levels within a band (either conduction or valence or both) are originated from spatial confinement of charged carriers achieved by sandwiching a low band-gap material in between two high band-gap materials as shown in Figure:1.2. AlGaAs/GaAs based QWIPs have

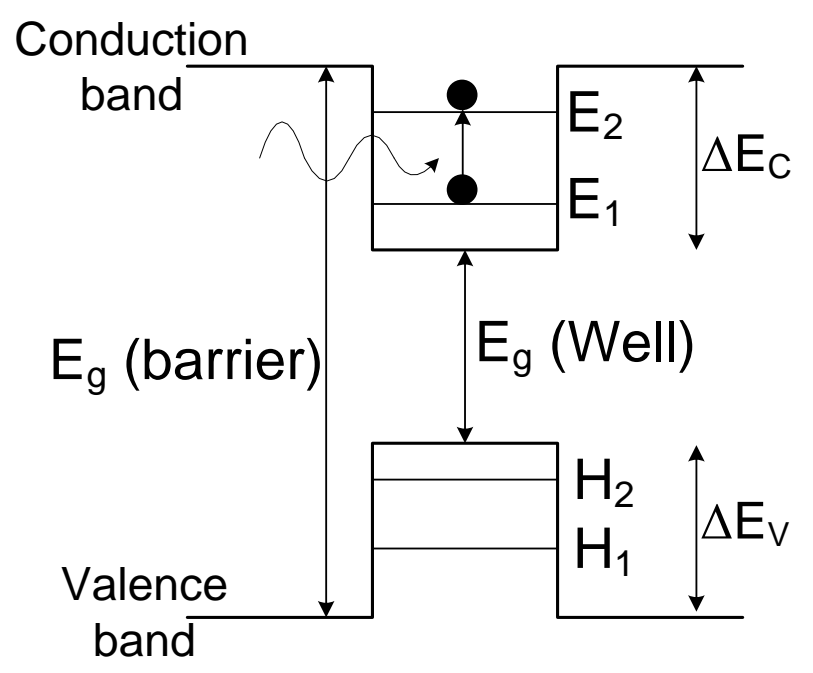

Figure: 1.2 Band structure of Quantum-well inter-sub-band absorption between electron levels $E_{1}$ and $E_{2}$ or levels $H_{1}$ and $H_{2}$ is schematically shown. 
significant advantages over $\mathrm{HgCdTe}$ detectors. Mature GaAs growth and processing techniques lead to high uniformity and excellent reproducibility, which allow the fabrication of large-area low-cost detector arrays. The ability to precisely control peak detection wavelength and spectral bandwidth by varying device and material parameters such as QW width and barrier composition and thickness enables the fabrication of multicolor detectors and modulators and also allows monolithic integration of these detectors with high-speed electronics.

For a rectangular potential well with infinite depth, with parabolic bands assumed, the energy levels are simply given by

$$
\mathrm{E}_{\mathrm{j}}=\left(\frac{\hbar^{2} \pi^{2}}{2 m^{*} L_{w}{ }^{2}}\right) j^{2}
$$

where $L_{w}$ is the width of the quantum well, $m^{*}$ is the effective mass in the well, and $j$ is an integer. The transition energy between the two sub bands is thus

$$
E_{1}-E_{2}=\left(3 \hbar^{2} \pi^{2} / 2 m^{*} L_{w}{ }^{2}\right)
$$

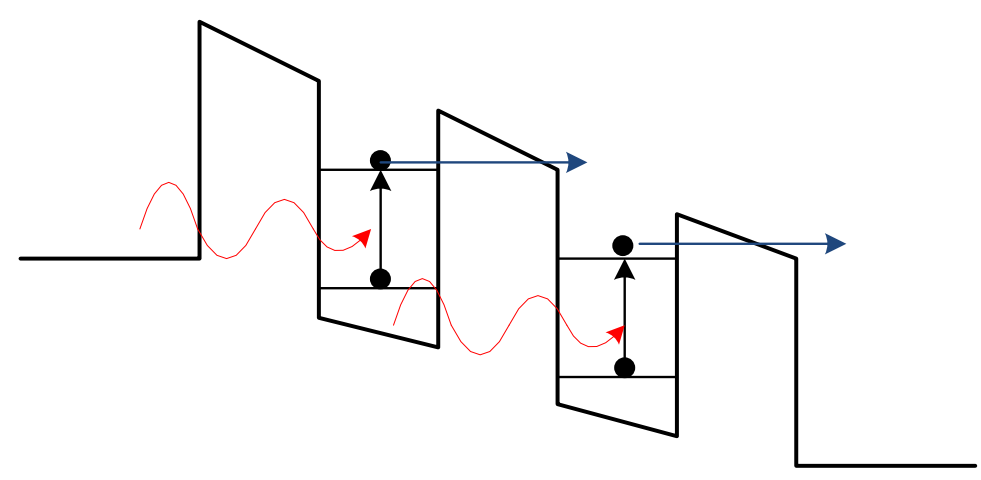

Figure: 1.3 Bound-to-bound inter-sub-band transition followed by tunneling out of the well 
This transition has a large dipole matrix element $\left\langle z=16 L / 9 \pi^{2}\right\rangle$. The oscillator strength for the quantum well with infinitely high barriers is given by [9]

$$
f \equiv \frac{2 m^{*}}{\hbar^{2}}\left(E_{2}-E_{1}\right)\langle z\rangle^{2}
$$

Since oscillator strength determines absorption of photons with that particular energy, by varying the quantum well width, the absorption wavelength can be tuned.

The QWIP demonstrated by Levine et al .[2] was based on quantum wells containing two bound states. By absorbing infrared signals, intersubband transition occurs from the bound ground state to the bound excited state. The photoexcited electrons then tunnel out of the well (Figure:1.3) and are transported by the electric field over a distance $\mathrm{L}$ which is the mean free path for the photo-excited carriers for recapture back into the quantum wells.

As most of the oscillator strength is concentrated in the bound-to-bound state transition, the absorption probability from the ground state to the continuum above the top is very weak [10]. However, by decreasing the size of the quantum well, the strong oscillator strength of the excited bound state can be pushed up into the continuum, leading to a strong bound-to-continuum state absorption. This has been shown in Figure:1.4. This extended state structure has the major advantage that the photoexcited electron can escape from the quantum well without tunneling through the energy barrier. The bias voltage required for the photoelectron to escape from the well therefore is low, 


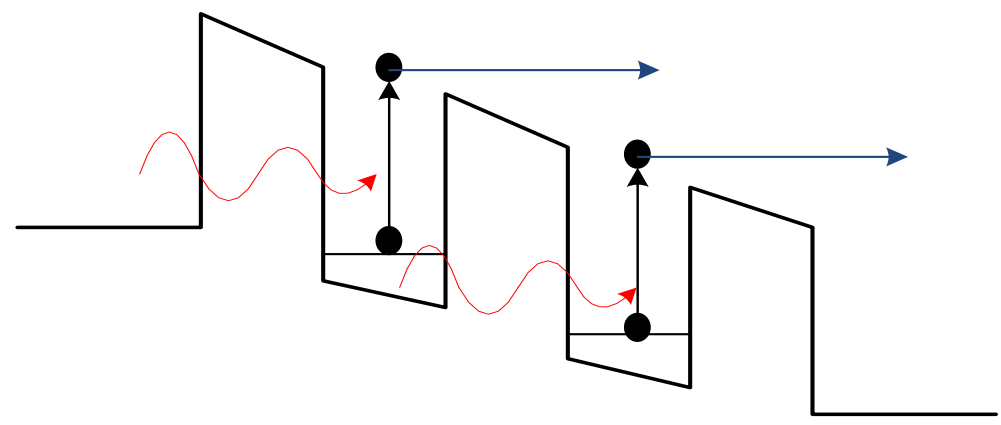

Figure: 1.4 A GaAs/AIGaAs QWIP structure which employs bound-to-continuum.

which results in reduced dark current. Also this allows the deployment of thicker barriers that can suppress the ground state tunneling probability and hence reduce the dark current further [11-15]. The low required bias voltage and reduced dark current make bound-tocontinuum structure particularly attractive for low power applications. The bias voltage required to transport free photoexcited carriers in bound-to-continuum QWIP is less than $5 \mathrm{~V}$, whereas in bound-to-bound QWIP, it is $9 \mathrm{~V}$ [10]. For same temperature and crosssectional area, the dark currents measured for a bound-to-continuum and a bound-tobound QWIP were $1 \times 10^{-12} \mathrm{~A}$ and $1 \times 10^{-4} \mathrm{~A}$ respectively [10], which represents a significant improvement.

\subsection{Asymmetric Quantum-well Structure}

One way to introduce asymmetry is to use multiple layers of different material of similar material with different compositions in the well region. This produces a stepped QW. In this study we have extensively investigated $A l G a A s / \mathrm{In}_{\mathrm{x}} G a_{(1-x)} A s / \mathrm{In}_{\mathrm{y}} \mathrm{Ga}_{(1-}$ y) As/AlGaAs QW (shown in Figure:1.5), which provides some additional design to simple rectangular wells, where only the well width and barrier height can be varied. But 
using such asymmetric well, the geometry and composition of the materials for both wells and barriers can be varied to tune the wavelength [10]. Moreover dipole matrix elements of transitions for odd-to-odd or even-to-even quantum number disappear in rectangular quantum well. On the other hand, Helgesen et al [16] found that the double well structure can achieve a significant enhancement of the dipole matrix element, due to the double maximum shape of the ground-state wave function. Thus, stepped quantum well structures are likely to provide the flexibility of adjusting both the energy levels involved in transition and the oscillation strength.
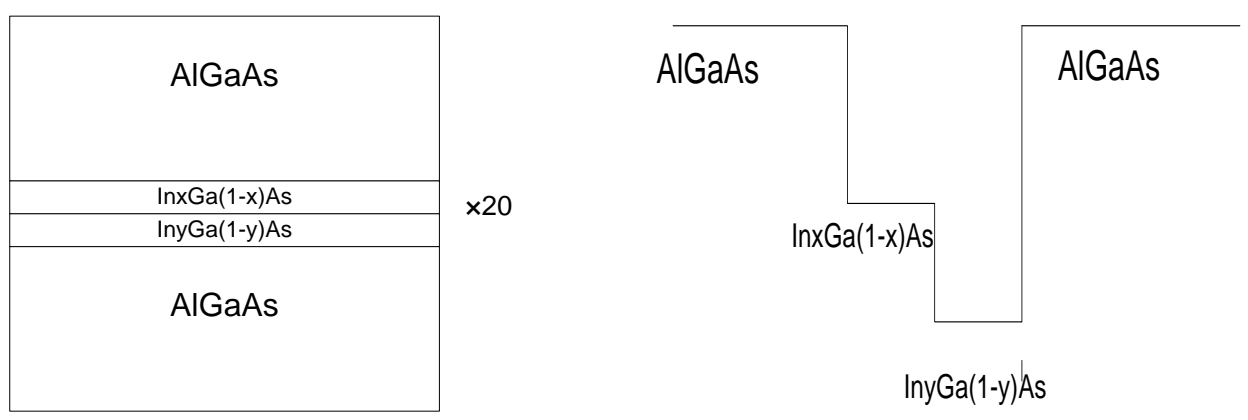

\section{Figure: 1.5 AIGaAs /In $\mathrm{n}_{\mathrm{x}} \mathrm{a}_{(1-\mathrm{x})} A s / \mathrm{In}_{\mathrm{y}} \mathrm{Ga}_{(1-\mathrm{y})} \mathrm{As} / \mathrm{AIGaAs}$ QWIP structure}

In the structure studied, the well is doped with $2 \times 10^{18} / \mathrm{cm}^{3}$ (not in step). Pan et al [17] showed that using such doping asymmetry, a large number of intersubband transitions which are forbidden in uniformly doped wells can be achieved. To enhance the speed of the detector, the material with high mobility (InGaAs) is used as the well material. However, using indium in the quantum well material means that strain is incorporated in the structure as GaAs and InGaAs are not lattice-matched. While InGaAs is grown epitaxially on GaAs or AlGaAs, first few atomic layers will remain strained to match the previous layer. Once a certain thickness is exceeded, defects will be introduced 
in the structure. This will undermine the carrier mobility and therefore the layer thickness should be carefully optimized. The procedure to calculate this optimum thickness is described in later section. Taking this into consideration indium composition is varied from $1.5 \%$ to $18 \%$ and $\mathrm{Al}$ composition is varied from $0-40 \%$ obtain the desired $8-10 \mu \mathrm{m}$ detection wavelength.

The voltage tunability is achieved by incorporating one additional potential well in a period as shown in Figure: 1.6. This approach is based on transferring electrons between the ground states of coupled QWs under an applied bias. Each period of the multiple quantum well (QW) structure consists of a left QW coupled (LQW) to a right QW (RQW) through a thin AlGaAs barrier to facilitate tunneling. The double QW structure is separated from the next set of coupled wells by a thicker AlGaAs barrier, as shown in Figure:1.6. In this work only one well has been stepped, although both wells can be made stepped. At zero bias, the lowest two states in the RQW have an energy difference of $\Delta E_{R Q W}$ while those in the LQW are separated by $\Delta E_{L Q W}$. These structures are usually designed to have a bound to continuum transition (B-C) in the RQW and either a bound to bound (B-B) or a bound to continuum transition in the LQW. In Figure:1.6, B-C and B-B transitions in the RQW and the LQW, respectively. Under positive bias shown in Figure: 1.6(b), electrons are transferred from the ground state of the LQW to the ground state of the RQW through tunneling. When infrared radiation is coupled into this detector, electrons are excited from the ground state of the RQW to the continuum of states above it. These photoelectrons are 

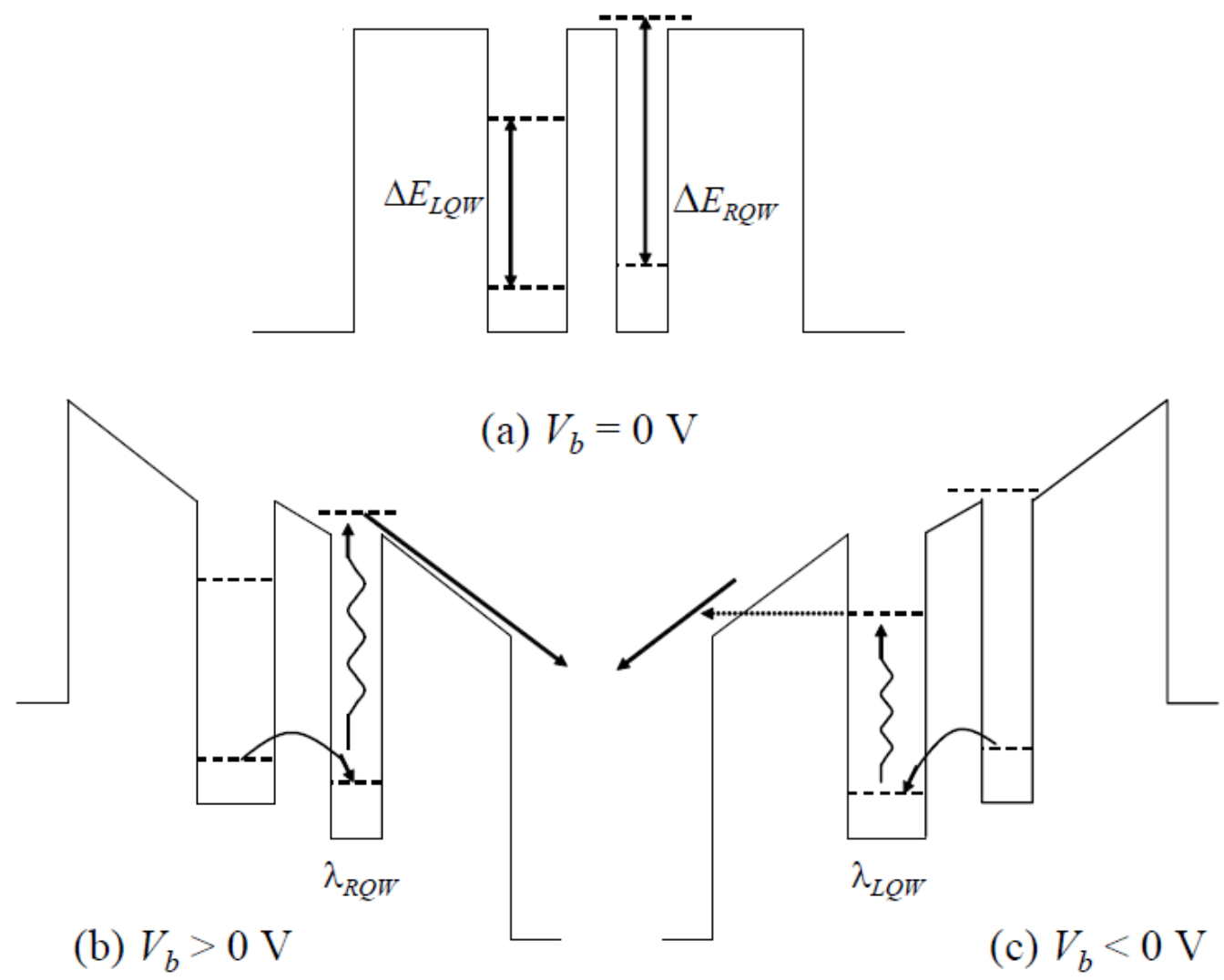

Figure: 1.6. Energy band diagram of a double quantum well structure for voltage tunable photodetector: (a) in equilibrium, (b) positive voltage applied and (c) negative voltage applied.

Thus, the structure is expected to detect radiation at a wavelength of $\lambda_{R Q W}=h c / \Delta E_{R Q W}$ for positive bias. As illustrated in Figure: 1.6(c), under negative bias electrons tunnel from the RQW to the LQW and lead to photodetection at $\lambda_{L Q W}=h c / \Delta E_{L Q W}$. It is important to note that under negative bias, the LQW photoexcited carriers must tunnel through a triangular barrier before contributing to photocurrent in detectors with a B-B LQW transition. The electron transfer process was first observed by Choi et al. [18] in multiple quantum well structures with alternately doped QWs. Subsequently, this mechanism was 
demonstrated in optical modulators [19-21]. The success of voltage tunable detector depends on careful optimization of the structure dimension such that when coupled to infrared radiation electron tunneling rate through the intermediate barrier is favorable to absorption. For example under positive bias, electron tunneling lifetime from left to right well must be significantly smaller compared to the electron transition lifetime from the ground to the excited state of the LQW. The optimization and calculation methodology used to achieve this has been detailed in chapter 2 .

\subsection{Thesis Outline}

This thesis presents investigation on photon absorption in asymmetric quantum well for infrared photodetectors (QWIP) to facilitate absorbing normal incidence. The motivation of this work is twofold: first to develop analytical/ mathematical model to analyze single or multiple quantum well structures to quantify photon absorption efficiency; and second is to optimize and characterize a complete quantum well photo detector through simulation. The physical model developed in this work is based on non-equilibrium Green's function (NEGF), and Fermi's golden rule and quantum mechanical wave impedance concept. Unlike previously reported works that assume broadening of energy states due to inter subband transition for calculating absorption coefficient, this model quantifies broadenings resulting from both optical absorption and tunneling. Results have been presented for $\mathrm{Al}_{x} \mathrm{Ga}_{1-x} \mathrm{As} / \mathrm{GaAs} / \mathrm{In}_{y} \mathrm{Ga}_{1-y} \mathrm{As}$ system which confirms that asymmetry in a QW breaks the symmetry or parity of the bound state wavefunctions and hence 
facilitates transition among certain energy states, which are otherwise forbidden by the selection rule. Assuming TM polarization of light, it has been demonstrated that stepped QW structures can in fact have improved absorption coefficient due to this symmetry breaking. The approach presented here can be extended to calculate the response of any arbitrary asymmetric well to both TE and TM polarization.

Chapter 2, titled "Optical Absorption in Quantum Wells" details the physical mechanism detecting infrared radiation and quantifying various performance parameters like oscillator strength, absorbance and responsivity. The chapter details the mathematics to calculate the eigenstates and oscillator strengths that quantifies transition probabilities or in other words coupling strengths among those states. In this context the quantum mechanical tunneling from a bound to bound state is discussed. The reasoning behind choosing the materials to produce asymmetric well structure is then discussed. The detailed mathematical reasoning behind symmetric wells' failure to absorb normal incidence is also presented here. Finally the chapter discusses quantitatively how voltage tunability can be achieved.

In Chapter 3, titles "Quantifying Inter-subband Transition in Asymmetric Quantum Wells", the calculation approach and algorithm used to quantify optical absorption in asymmetric QW is represented. The chapter details the modeling framework developed to analyze the structures and how it quantifies the performance parameters and the impact of different structural dimensions (like doping concentrations, depths and widths of well, step and barriers). In this work the idea of quantum mechanical wave impedance concept has been used to calculate the Eigen states, along 
with non-equilibrium Green's function to calculate the tunneling probabilities and oscillator strength. The procedure is inherently simpler as it does not require any matrix manipulation, yet computationally efficient. This model takes into account and calculates the broadening of the energy states due to absorption and tunneling, which was only assumed in previously reported works while calculating absorption. So this model gives a complete and accurate result in terms of absorption. The chapter concludes describing how the structure is optimized to be tuned to the desired absorption spectra with acceptable responsivity.

Chapter 4, is titled "Analysis of Asymmetric Quantum Wells for Infrared Photodetection", and describes the investigated structures. The calculated results obtained though the developed model described in chapter 3 is presented here. Physical consistency of the results and their comparison with published works are also presented. The results obtained for different polarization of light for different structures are discussed here. The asymmetry of the structure aids the absorption of normal incidence light without changing the geometry of incident plane of the detector and thus facilitates the fabrication of large arrays of focal planes. One of the major contributions of the model developed here is to provide an efficient way to quantify the absorption rate of different light polarizations and thus enable effective optimization of the photodetector structure. Finally voltage tenability is demonstrated for a muti-quantum well structure. The potential advantages that can be achieved through the structure analyzed in this work is then outlined. 
The concluding remarks are provided in Chapter 5:'Summary and Concluding Remarks", which provides the research summary by briefly reviewing the photon absorption physics, along with the modeling framework, and obtained results. The chapter ends with some suggestions to current and future researchers as to what can be studied further in such structures. 


\section{CHAPTER 2}

\section{Optical Absorption in Quantum Wells}

The physical mechanism of infrared (IR) photo-detection specifically using quantum well structures is presented in this chapter. Like any other photodetection mechanisms, an IR photo-detector responds to incident electromagnetic radiation by generating electric current. Electrons (in some cases holes as well) in a suitable semiconductor material are excited from their lower energy states to higher ones in the conduction band where they can freely respond to applied electric field and flow through external detection circuitry. The amount of external current quantifies the "responsivity" of the photodetector to the incident electromagnetic wave of a particular wavelength. This chapter presents a comparative discussion on quantum well infra-red photodetection and interband photo detection schemes. Quantum wells usually facilitate detection of long wave length radiation as they exploits carrier transition within in the same band, e.g. conduction band. This chapter also details various quantum structures that can be used as photodetectors. In the following section (2.1), infrared photo-detection mechanism has been discussed along with material systems commonly used for the purpose. Section 2.2 details a quantum well infrared photodetector (QWIP), in terms of optical absorption process, performance parameters such as responsivity and efficiency. The considerations regarding materials and structural choices of this research work is presented in later 
sections which describes the superiority of asymmetric quantum well structures like InGaAs/AlGaAs that has been focused in the study presented here.

\subsection{Infrared Photodetection:}

Almost everything radiates energy in the form of heat, which is nothing but electromagnetic waves having frequency in the infrared region. Infrared photodetectors therefore does not require any source of electromagnetic radiation to detect an object and posses an inherent advantage over other detectors. The energy radiated by any object at a given temperature is determined by its emissivity, $\varepsilon$, which is defined as the ratio of energy it radiates to that by a perfect emitter, termed as blackbody. The emission spectrum of an object at temperature $T$ as a function of wavelength $\lambda$ is given by $L_{S}(\lambda, T)$

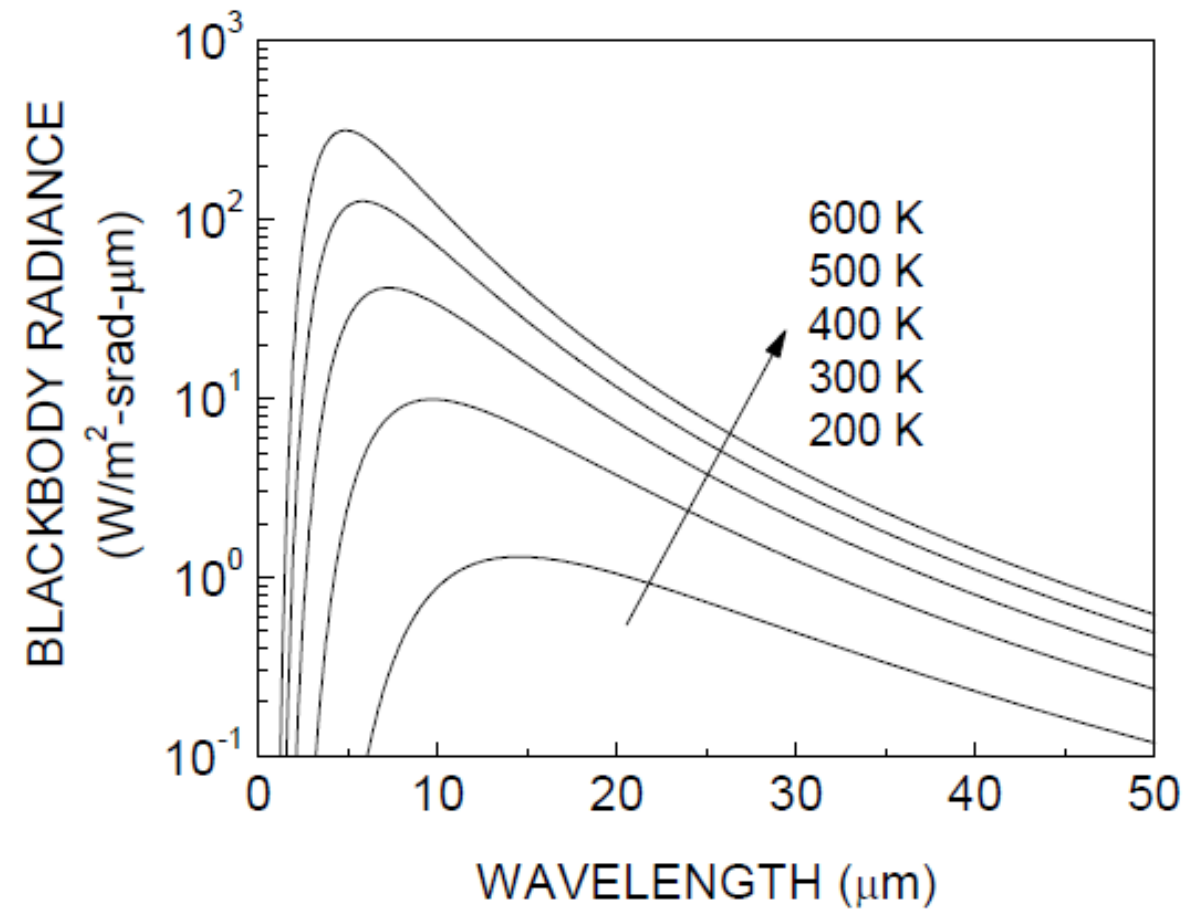

Figure: 2.1. Spectral radiance $L_{B B}$ of blackbodies at different temperatures $T$ [22]. 
$=\varepsilon(\lambda, T) L_{B B}(\lambda, T)$, where $L_{B B}$ is the spectral radiance of a blackbody at the same temperature and is calculated as [22]:

$$
L_{B B}(\lambda, T)=\frac{2 h c^{2}}{\lambda^{5}\left(e^{h c / \lambda k_{B} T}-1\right)}
$$

Here $h$ is the Plank's constant, $c$ is the speed of light in vacuum, $k_{B}$ is the Boltzman's constant, and $\mathrm{T}$ is the absolute temperature. Fig 2.1 shows the calculated values for $L_{B B}$ $(\lambda)$ at different temperatures.

As can be seen in from Figure: 2.1, generally emission peaks within 2-12 $\mu \mathrm{m}$ range. These blackbodies radiate most of their energy in the infrared range $(\lambda>0.7 \mu \mathrm{m})$. The peak emission at around room temperature $(300 \mathrm{~K})$ is found to be at $\sim 10 \mu \mathrm{m}$. The peak shifts to lower wavelengths as the blackbody temperature goes up. $\lambda m \approx 10 \mu \mathrm{m}$ for blackbodies at room temperature $T \approx 300 \mathrm{~K}$. Coincidentally, the transmittance of electromagnetic radiation through atmosphere becomes maximum at wavelengths 8-14 $\mu \mathrm{m}$ range (along with $2-5 \mu \mathrm{m}$ and $28-34 \mu \mathrm{m}$ range), which is known as atmospheric transmittance window [23]. This makes an infrared detector very powerful tool for observing distant objects at or around $300 \mathrm{~K}$. Figure: 2.2 show a biased two-terminal photoconductor with optical power Pin incident on an area $A$. The incident photon flux is $\varphi_{\text {in }}=P_{i n} / h v$, where $h v$ is the photon energy. The number of photons absorbed per unit time is $\eta \varphi_{i n}$, where $\eta$ is the quantum efficiency of the photoconductor that depends on absorption coefficient $\alpha$ of the device. Assuming each absorbed photon creates a photoelectron, the generation rate of photoelectrons per unit volume is $G=\eta \varphi_{i n} / A L$. In steady state, the photoelectron density is $n=G \tau=\eta \varphi_{\text {in }} \tau / A L$, where $\tau$ is the electron 
lifetime. When a voltage bias $V_{b}$ is applied across the detector, the photocurrent flowing between the two contacts separated by $L$ is

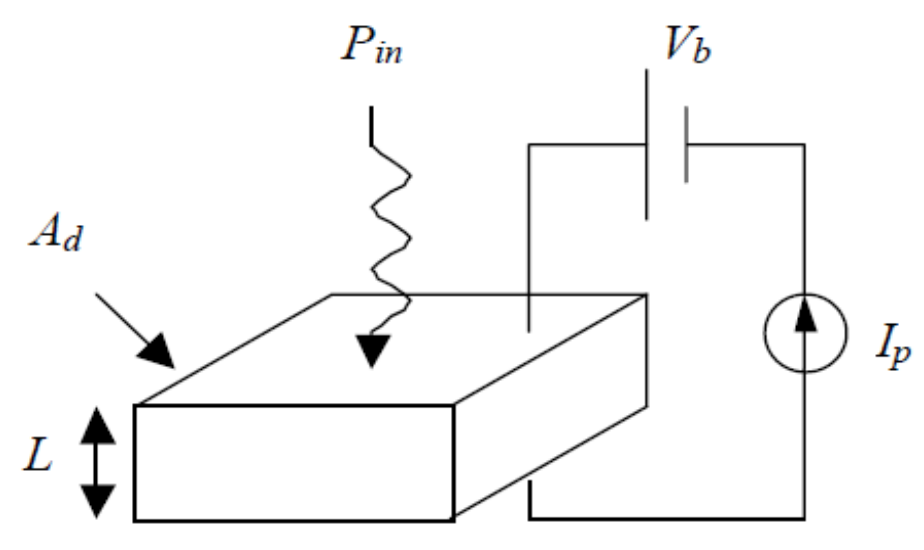

Figure: 2.2. Schematic of a photodetection mechanism, showing a phodetector biased and connected to external detection circuitry.

$$
I_{p}=e n A v_{d}=e \eta \varphi_{i n} \tau v_{d} / L=e \eta \varphi_{i n} g, \text { where } e \text { is the charge of an electron, } v_{d} \text { the }
$$

electron drift velocity, and $g=\tau v_{d} / L$ the photoconductive gain, defined as the ratio of the lifetime to the transit time $\left(L / v_{d}\right)$ across the detector and can be interpreted as the fraction of electrons contributing in photocurrent to the total photo-generated electrons. Detector responsivity $R$ is defined as the photocurrent $I p$ generated per unit incident optical power Pin and can be expressed in terms of quantum efficiency $(\eta)$, photoconductive gain $(g)$ and tunneling probability [24-25]:

$$
R=\frac{I_{P}}{P_{i n}}=\frac{e}{h v} \eta g T
$$

The mercury-cadmium-telluride, MCT (HgCdTe), which is an alloy of $\mathrm{HgTe}$ and $\mathrm{CdTe}$, is the only common material that can detect infrared radiation in both of the accessible atmospheric windows namely 3-5 $\mu \mathrm{m}$ and 8-12 $\mu \mathrm{m}$. Understandably $\mathrm{HgCdTe}$ 
is a common material in photodetectors used in a wide range of applications spanning from Fourier transform infrared spectrometers, military field specifically for night vision, remote sensing and infrared astronomy research. Although HgCdTe-based detectors operating at $3-5 \mu \mathrm{m}$ require cooling to temperatures near that of liquid nitrogen $(77 \mathrm{~K})$, to reduce noise due to thermally excited current carriers, $\mathrm{HgCdTe}$ based detectors enjoy much higher speed of detection and is significantly more sensitive than some of its cheaper competitors like Si-based bolometers [26]. However, as have been mentioned in the previous chapter, weak $\mathrm{Hg}-\mathrm{Te}$ and $\mathrm{Cd}-\mathrm{Te}$ bonds leading to bulk, surface and interface instabilities severely undermines the performance of MCT detectors [6-8]. Quantum well infrared photodetectors (QWIP), manufactured from III-V semiconductor materials such as GaAs, AlGaAs, InGaAs etc., have been investigated as potential alternative material systems. But as will be detailed in later sections, the performance of quantum well based photodetectors are limited by the fact that they require the use of complicated reflection/diffraction gratings to overcome certain polarization exclusion effects which impact responsivity.

\subsection{Traditional interband based detectors and intraband QWIPs}

It has been already mentioned that the materials used in both mid wavelength infra-red (MWIR) and long wavelength regions (LWIR) are mainly mercury cadmium telluride (MCT) and InSb. Usually these MCT based detectors utilize interband transition of carriers to absorb photons. The primary drawback of traditional MCT based technology is that it is soft, difficult to synthesis by any growth method and suffers from 
non-uniformity, making the fabrication of focal plane arrays a challenging and costly proposition. The band gap of MCT varies as a function of its mercury composition to $\mathrm{HgTe}$, which is a semimetal with a negative band gap, to CdTe with a band gap of 1.6 $\mathrm{eV}$. At a mercury composition of $80 \%$, the band gap attains a value of $1.6 \mathrm{eV}$, sufficient for detection of IR radiation up to $12 \mu \mathrm{m}$. In the low wavelength IR (LWIR), the high concentration of mercury is the reason for the great difficulty in achieving uniform focal plane arrays. As a result, GaAs based QWIPs, which absorbs radiation through interband or intra-subband transition, are emerging as dominating candidates in the range of 8-12 $\mu \mathrm{m}$ and also have achieved considerable commercial success [9]. The main reason behind the great interest in GaAs stems largely from the fact that it has mature material growth and device processing technologies. Much effort has been devoted to optimize the performance of devices operating in the 8-12 $\mu \mathrm{m}$ regime, most of which are based on photoconductive multi-quantum well structures, because of their high responsivity. However in the MWIR region, where the concentration of mercury needed to synthesize devices is low, MCT has been the main driving force and two-dimensional array technology has been developed based on both MCT and InSb compounds. Although, InSb does not suffer from material uniformity problems which trouble MCT, it also requires cooling while being operated at mid-IR region, as the band gap decreases with increasing temperature and this exacerbates the enhanced thermal generation always associated with temperature elevation. This limits device operating cut-off wavelength above $5 \mu \mathrm{m}$. Introduction of InAlSb (10\% Al) blocking layers has successfully raised the temperature at which images can be obtained from InSb arrays ( $256 \times 256$ pixels) to 
130K. Use of all InAlSb $(3.5 \% \mathrm{Al})$ devices can raise this operating temperature still further to $150 \mathrm{~K}$ while keeping the operating wavelength below $5 \mu \mathrm{m}[10]$.

One of the drawbacks of QWIP detectors is their lack of ability to absorb normal incidence originating from the polarization selection rules [11], that has been extensively discussed later. This necessitates additional processing in the form of gratings or polishing at $45^{\circ}$ to achieve coupling of the light such that normally incidence radiation can have field component in desired direction. The internal quantum efficiency is also typically less than $20 \%$, compared to more than $70 \%$ achievable with interband detectors due to the smaller number of carriers available to take part in transitions. Increasing the doping can enhance quantum efficiency [12] but this necessarily raises the Fermi energy and thus quantum efficiency is increased at the expense of a relatively large dark current and hence increased noise for a given operating temperature. For a detector responding in the range $3-5 \mu \mathrm{m}(8-9.5 \mu \mathrm{m})$, cooling to temperatures down to $100-120 \mathrm{~K}(70-77 \mathrm{~K})$ is typically necessary in order to reduce dark currents to sufficiently low levels. The dominant mechanism of dark current generation in QWIPs is longitudinal optical phonon excitation of carriers because of very strong coupling in III-V materials. The most obvious way of minimizing the dark current is thus to decrease the detector temperature. It is also evident that cooling requirements become more severe for longer cut-off wavelengths. The temperature at which background limited performance (BLIP) is achieved is a useful figure of merit to compare detector operating temperature performance. A BLIP detector is defined as one in which the dark current is less than or equal to the background ( $300 \mathrm{~K})$ photocurrent $\left(180^{\circ}\right.$ field of view, with a cooled sample). 
It is only in the last few years that sufficient advances in epitaxial growth, synthesis and design of strained GaAs and InP quantum well structures have been made, and this has started to challenge the pre-eminence of MCT and InSb in the MWIR region. Along with the uniformity of growth and processing, which are essential for focal plane arrays, the advantages that these materials potentially offer includes high speed switching for free space communications applications [13].The combination of relatively short wavelengths and the high barriers achieved in double barrier quantum well (DBQW) structures also enables high temperature operation for moderately doped QWIP structures $[14,15]$. Apart from these materials, other technologies such as antimonide-based compounds are also being intensively researched $[16,17]$ and are showing real potential for the MWIR detection. Nevertheless this technology is still not matured enough to be a competitor of either GaAs or InP.

\subsection{Quantum well Infrared Photodetector (QWIP)}

Quantum well infrared photodetectors (QWIP) were originally built on GaAs/al ${ }_{x} \mathrm{Ga}_{1-x} \mathrm{As}$ lattice matched multiple quantum wells [18]. A QW is formed in GaAs when it is sandwiched between two layers of AlGaAs. Confinement of carriers in the QW leads to quantization of energy levels, as shown in Figure:2.3. Signatures of these energy levels have been observed in transport [27] and interband absorption experiments [28]. By varying the $\mathrm{Al}$ molar ratio $x$ in the barriers, intersubband absorption at a wide range of wavelengths was demonstrated. For instance, using a small $x$ of 0.07 , a far infrared QWIP detecting $27.1 \mu \mathrm{m}$ wave was designed [29]. Detection was also reported at the other extreme of infrared spectrum, namely at $2.9 \mu \mathrm{m}$, using $x=1$ [30]. Moreover energy level 
separations of the order of $100 \mathrm{meV}$ can be achieved with proper tuning of well width and depth.

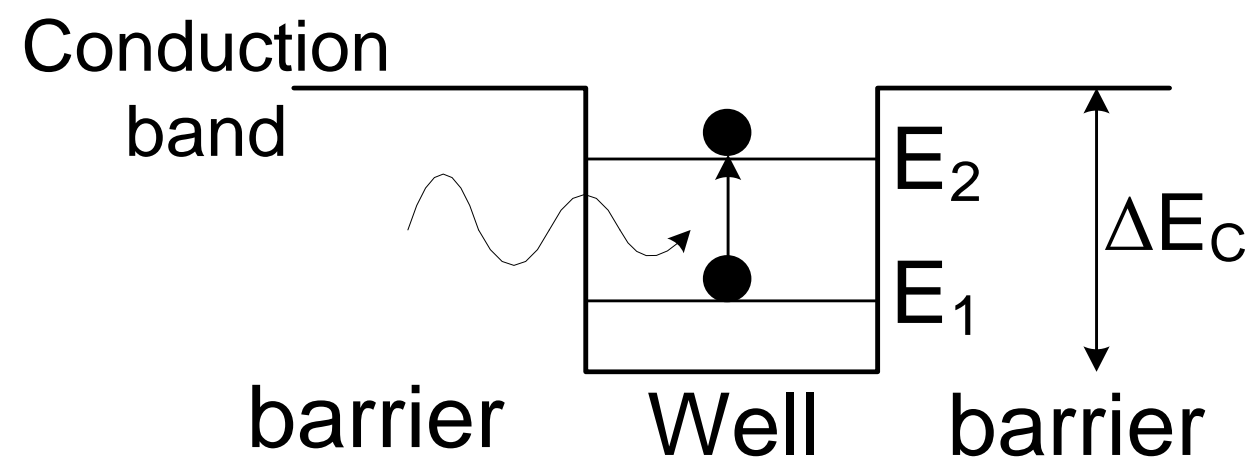

Figure: 2.3 Schematics of conduction band profile of a quantum well structure absorbing radiation

\section{Optical Absorption in QWIPs: different transitions}

Absorption of infrared radiation excites electrons from the ground state to an excited state of a quantum well. A QW structure is particularly suitable infrared radiation or low energy photons as the energy separations among the quantized states can be made much smaller compared to that of the band gaps of most commonly used semiconductors. Since intraband transition resulting from infrared absorption occurs entirely within conduction band, electron-hole pairs are not created and hence QWs must be doped (in this context $n$-doped) to ensure sufficient carrier concentration required for producing detectable photo-current.

In order to quantify the absorption in a QWIP, one needs to calculate the electron transition rate among the bound states of the quantum well. The detail of calculating the energy eigen states and the wave functions associated with them for a generalized 
quantum well structure will be presented in chapter 3. However for illustrating the calculating transition rate, let us assume a rectangular potential well with infinite depth. Then with parabolic bands assumed, the energy levels are simply given by

$$
\mathrm{E}_{\mathrm{j}}=\left(\frac{\hbar^{2} \pi^{2}}{2 m^{*} L_{w}^{2}}\right) j^{2}
$$

where $L_{w}$ is the width of the quantum well, $m^{*}$ is the effective mass in the well, and $j$ is an integer. The transition energy between the two sub bands is thus

$$
E_{1}-E_{2}=\left(3 \hbar^{2} \pi^{2} / 2 m^{*} L_{w}^{2}\right)
$$

One of the most important parameter quantifying the detector performance, is responsivity $R$, which is given by $R=\frac{e}{\hbar \omega} \eta G T$. Assuming $G$ and $P$ to be constants in $\omega$, the spectral shape of $R(\omega)$ will be determined by the functional form of $f(\omega)$. It has been found that the product of $G$ and $P$ is approximately constant for bound-to-continuum detectors at low bias. [30,31]. With this assumption, the normalized responsivity $N_{R}$, defined as $C f(\hbar \omega) / \hbar \omega$ for some normalization constant $C$, will give the spectral line shape of $R$. Its maxima determine the responsivity peak wavelength.

\subsection{Inter-subband absorption between bound states}

Different types of quantum well structures are used for intersubband absorption as discussed in the following sections.

\section{A. Rectangular quantum wells}

1. $\mathbf{G a A s} / \mathbf{A l}_{\mathbf{x}} \mathbf{G a}_{(1-\mathbf{x})} \mathbf{A s :}$ Intersubband absorption results from transitions between energy levels within the same conduction or valence band, as schematically represented 
in Figure: 2.4. These levels are originated from the spatial localization of charge carriers or electrons introduced by quantum wells, that is formed when a low-band-gap material (e.g., GaAs) is sandwiched by a higher-band-gap semiconductor (e.g., $\left.\mathrm{AI}_{\mathrm{x}} \mathrm{Ga}_{(1-\mathrm{x})} \mathrm{As}\right)$. For infinitely high barriers and parabolic bands, the energy levels in the well are simply given by [22] equation 2.3 , whereas the intersubband transition energy between the lowest and first excited state is thus given by equation 2.4. Furthermore this transition has integrated absorption strength of

$$
\int_{0}^{\infty} \alpha(v) d v=\left(\frac{\rho_{c} N_{w} e^{2} h f}{4 \in_{0} m^{*} c n_{r}}\right)\left(\frac{\sin ^{2} \theta^{\prime}}{\cos \theta^{\prime}}\right)
$$

where $\rho_{c}=N_{D} L_{w}$ is the two-dimensional density of carriers in the well, $N_{D}$ is the three-.

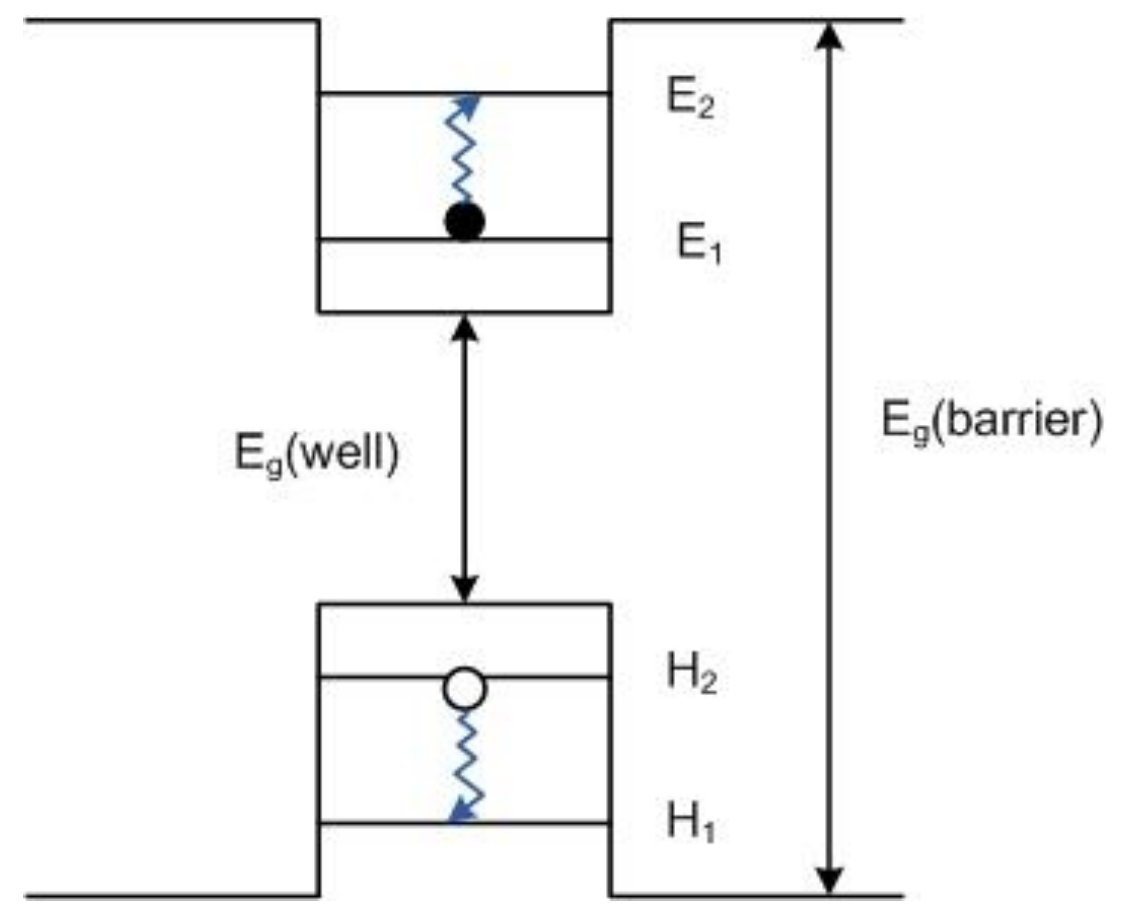

Figure: 2.4. Band structure of quantum-well (depths $\Delta E_{c}$ and $\Delta E_{v}$. Intersubband absorption between electron levels $E_{1}$ and $E_{2}$ or hole levels $H_{1}$ to $H_{2}$ is schematically shown. 
dimensional carrier density, $N_{w}$ is the number of doped wells, $n_{r}$ is the index of refraction, $\theta^{\prime}$ is the angle between the direction of the optical beam and the surface normal (inside the medium), and $f$ is the oscillator strength.

This oscillator strength, which is proportional to the energy separation of the concerned eigenstates, is very large (near unity) for a quantum well with infinitely high barriers and can be expressed as [22]

$$
f \equiv \frac{2 m^{*}}{\hbar^{2}}\left(E_{2}-E_{1}\right)\langle z\rangle^{2}=\frac{256}{27 \pi^{2}} \cong 0.96
$$

where $z$ is the direction normal to the quantum well. Thus, by changing the quantum-well width $L_{w}$ this intersubband transition energy can be varied over a wide range from the short wave infrared SWIR $(\lambda \sim 2 \mu \mathrm{m})$, the midwave infrared MWIR $(\lambda \sim 4 \mu \mathrm{m})$, through long-wave LWIR $(\lambda \sim 10 \mu m)$ and into the very long-wave VWIR spectral regions ( $\lambda>14 \mu m$ ). It should be noted from equations ( 2.5) and (2.6) that since the oscillator strength only has a component along $z$, the optical electric field must also have a component parallel to $z$ in order to induce an intersubband absorption; thus, normal incidence radiation [i.e., $\theta^{\prime}=0$ in Eq. (2.5)] will not be absorbed. Therefore different optical coupling techniques that include ingenious gration structures, have been deployed. Among many proposed schemes, West and Eglash [22] used a Brewsters' angle ( $\left.\theta_{B}=73^{\circ}\right)$ geometry in order to give a large component of the optical electric field along the growth (i.e., z) direction. A $5 \%$ absorption was measured at $\lambda=8 \mu \mathrm{m}$, corresponding to an oscillator strength near unity. Levine et al [10] reported the use of a multiple-pass waveguide geometry that increased the net intersubband absorption (Figures. 2.5). 


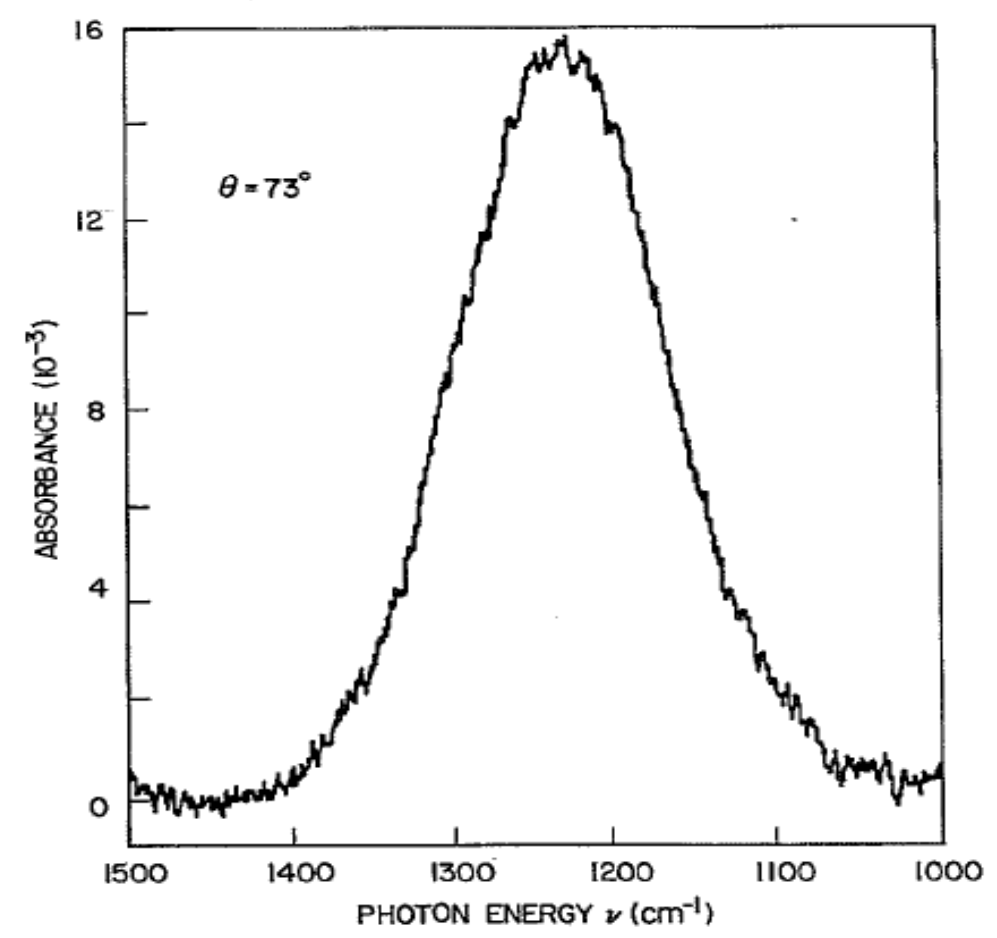

Figure: 2.5 Measured bound-to-bound QWIP intersubband absorbance=-log (transmission) vs photon energy at Brewster's angle $\theta_{B}=73^{\circ}$ [10].

and 2.6) by approximately two orders of magnitude, thus permitting accurate measurements of the oscillator strength, the polarization selection rule, and the line shape even for single quantum wells [23-27]. This have been shown in Figures: 2.5 and 2.6.

The polarization selection rule $\alpha \propto \cos ^{2} \phi$ (where $\phi$ is the angle between the electric field and the z direction) was found to approximately hold as shown in Figure. 2.7 where the absorption vanishes at $\phi=90^{\circ}$ (i.e., for light polarized in the plane of the quantum wells). This selection rule can also be tested by keeping the light polarized in the plane of incidence (i.e., $p$ polarization), but varying the angle of incidence $\theta$. The absorption can be calculated from $\alpha \propto T(\theta) \sin ^{2} \theta^{\prime} / \cos \theta^{\prime}$, where $T(\theta)$ is the transmission factor into the semiconductor and $\theta^{\prime}$ is the angle of incidence inside the 
material. This curve is plotted in Figure. 2.7 and is also in excellent agreement with experiment.

Detailed measurements and analyses of the intersubband absorption peak wavelength position $\lambda_{p}$ and spectral width have been done as a function of temperature, well width, barrier height (e.g., $\mathrm{AI}_{\mathrm{x}} \mathrm{Ga}_{(1-\mathrm{x})} \mathrm{As}$ composition), and doping $\mathrm{N}_{\mathrm{D}}$ and are reported in Ref [28-36]. It is found that, for high doping density $\left(\mathrm{N}_{\mathrm{D}}>10^{18} \mathrm{~cm}^{-3}\right)$, the peak absorption shifts to higher energy, which is accompanied by linewidth broadening, and a linear increase in oscillator strength with doping (i.e., $f \propto N_{D}$ ). It has been also shown that the shift in intersubband energy had a large contribution from the collective plasma oscillation (i.e., the depolarization shift) and a smaller contribution from the exciton like shift.

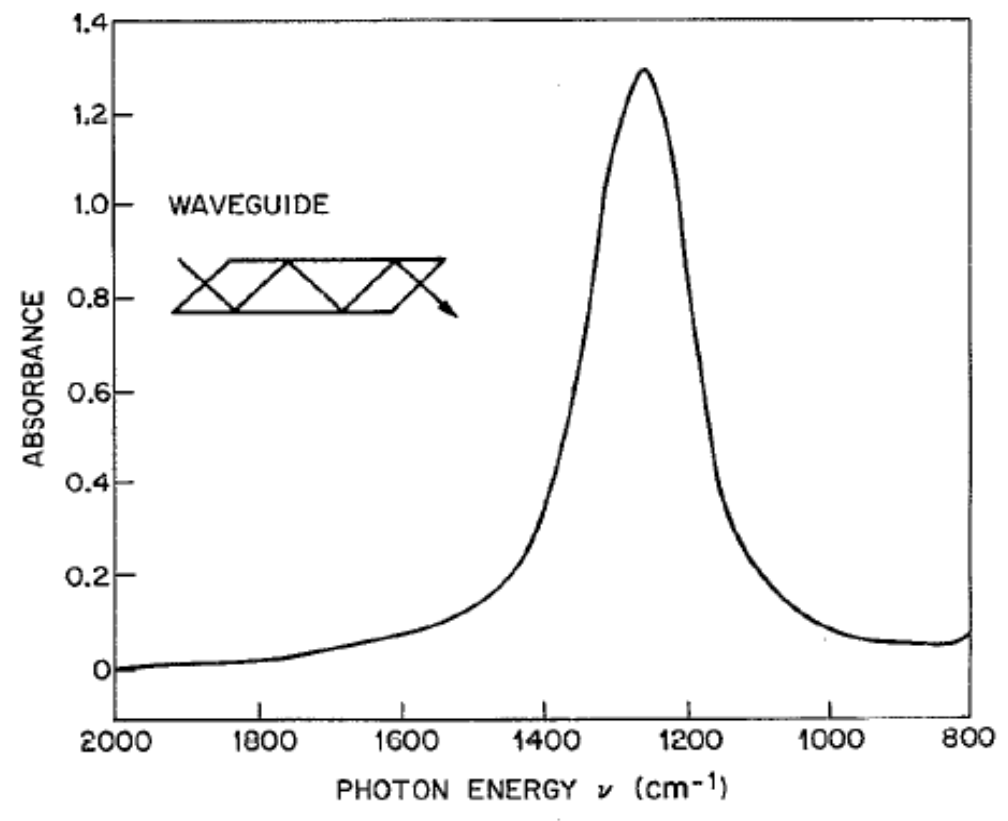

Figure: 2.6 Measured QWIP absorbance vs photon energy for the multipass waveguide geometry shown in the insert [10]. 
In addition to these doping shifts, there the peak wavelength and linewidth also changes with temperature $[37,38,39-41]$. As the temperature is lowered below room temperature down to cryogenic temperatures, a small $(\Delta v \sim 3 \%)$ decrease in the peak wavelength and linewidth is found. However, the oscillator strength remains constant so that the decrease in $\Delta v$ ( $\sim 75 \%$ of the room temperature value) is accompanied by an increase in the peak absorption [42] (by a factor 1.3). Regarding the temperature shift, Manasreh et al. [33] found that in order to explain this temperature dependence one must include the collective plasma, excitonlike, Coulomb and exchange interactions as well as nonparabolicity and the temperature dependencies of the effective mass and band gaps. It was also found that the intersubband absorption spectral linewidth depended significantly on the dopant impurity scattering.

Modulation-doped samples $[43,44]$ which separated the dopant from the quantum well by doping the barriers gave substantially narrower spectra. For example, Dupont $e t$ al [42] found that $\Delta v / v=4 \%$ was obtained for samples doped in the center of the barrier, to be compared with $\Delta v / v=8 \%$ for well-doped QWIPs. It is also worth mentioning that the intersubband absorption strength of GaAs grown directly on $\mathrm{Si}$ substrates has been reported [45] to be comparable to that grown on GaAs substrates. Such GaAs/Si QWIPs might be advantageous for large imaging arrays because there would be no thermal mismatch with a Si multiplexer. However, the question of the thermal cycling stability of the epilayer needs further study. 


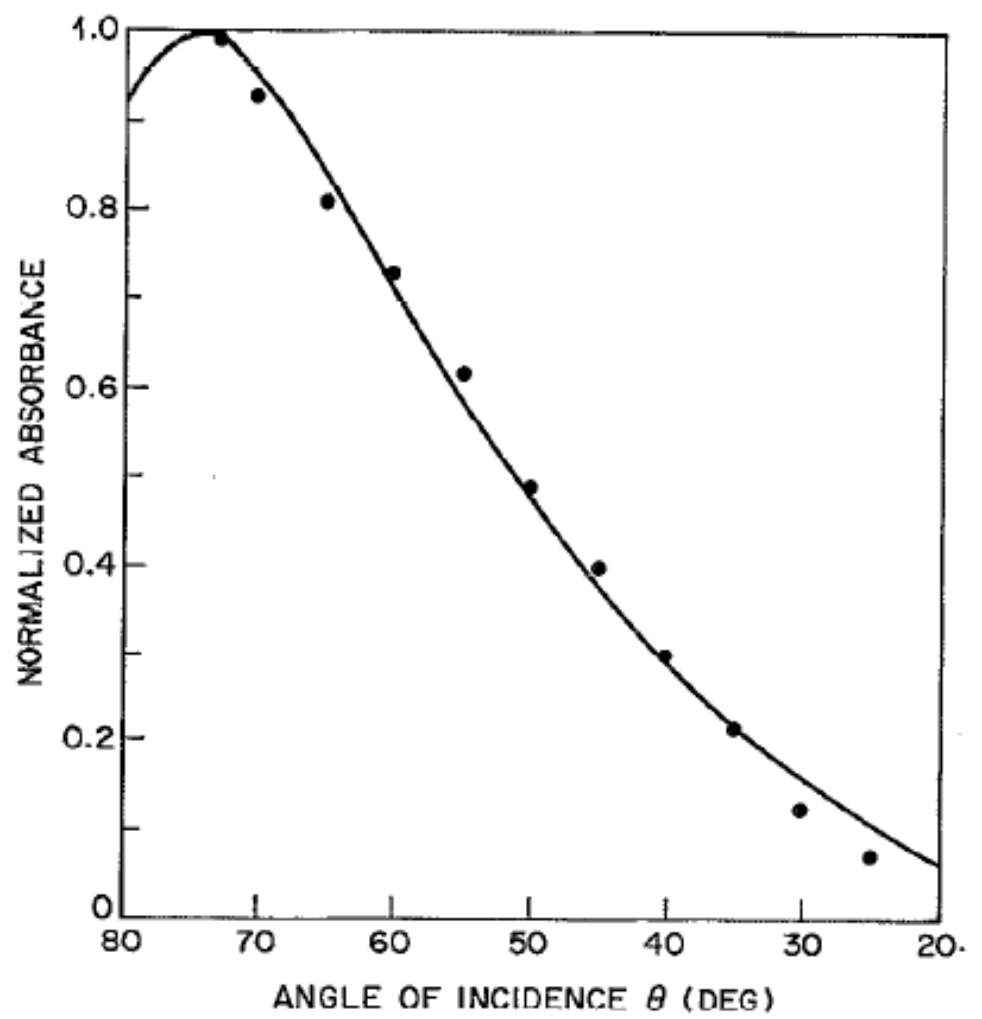

Figure: 2.7 Measured QWIP absorbance vs angle of incidence $\theta$ (dots), and theoretical dependence (solid curve)[10].

2. $\mathbf{I n}_{\mathrm{x}} \mathrm{Ga}_{(1-\mathrm{x})} \mathrm{As:}$ : In addition to the $\mathrm{GaAs} / \mathrm{Al}_{\mathrm{x}} \mathrm{Ga}_{(1-\mathrm{x})} \mathrm{As}$ materials system, intersubband absorption measurements and theoretical calculations on $\ln _{x} \mathrm{Ga}_{(1-\mathrm{x})}$ As multiquantum-well structures have also been investigated [46,47-52]. The doping $N_{D}$, well width $L_{w}$, and polarization dependence of the intersubband oscillator strength $f$ and peak position have been measured by Asai and Kawamura [53, 54] and Lobentanzer et al. [55] for $\mathrm{In}_{0.53} \mathrm{Ga}_{0.47} \mathrm{As} / \mathrm{In}_{0.52} \mathrm{Al}_{0.48} \mathrm{As}$ grown lattice matched on InP substrates. They find that $f$ is proportional to $N_{D}$ and that as the Fermi level is raised above the second bound level, both the $E_{1}$ to $E_{2}$ and the $E_{2}$ to $E_{3}$ transitions can be observed. By varying $L_{w}$ from 35 to $200 \AA$, the peak of the absorption could be shifted from $\lambda_{p}=4.1 \mu \mathrm{m}$ [the $\left(E_{2}-E_{l}\right)$ transition] 
to $\lambda_{p}=13.9 \mu \mathrm{m} \quad$ [the $\left(E_{4}-E_{3}\right)$ transition]. By using highly strained $\mathrm{In}_{0.66} \mathrm{Ga}_{0.33} \mathrm{As} / \mathrm{In}_{0.30} \mathrm{Al}_{0.70} \mathrm{As}$ multiquantum wells [56] grown on InP, the intersubband peak could be shifted to a significantly shorter wavelength, $\lambda_{p}=3.1 \mu \mathrm{m}$. Very interesting intersubband absorption experiments have been done by Peng et al.[48] on strained InGaAs/InAlAs single and multiple quantum wells. They find, in agreement with their theory,[68] that both $s$ and $p$ polarizations are allowed when the full symmetry (i.e., including both strain and lack of inversion symmetry) of the quantum wells is taken into account.

Zhou et al.[49] have measured the absorption in $\operatorname{In}_{\mathrm{x}} \mathrm{Ga}_{(1-\mathrm{x})} \mathrm{As} / \mathrm{In}_{0.40} \mathrm{Al}_{0.60} \mathrm{As}$ as a function of strain and composition for $0 \leq x \leq 0.15$. For $L_{w}=50 \AA$ they find that the intersubband transition varies from 6 to $7 \mu \mathrm{m}$. The $\operatorname{In}_{0.15} \mathrm{Ga}_{0.85} \mathrm{As} / \operatorname{In}_{0.35} \mathrm{Al}_{0.65} \mathrm{As}$ composition was studied in more detail by Shakuda and Katahama [50] who varied $N_{D}$ and $L_{w}$. They find that the transition energy increases with doping as expected due to the depolarization shift and that the $\left(E_{2}-E_{1}\right)$ and $\left(E_{3}-E_{2}\right)$ transitions both shift to lower energy [from 200 to $90 \mathrm{meV}$ for $\left.\left(E_{2}-E_{l}\right)\right]$ with increasing $L_{w}$ in good agreement with their theory.

Lattice-matched $\operatorname{In}_{0.53} \mathrm{Ga}_{0.47} \mathrm{As}$ quantum wells and InP barriers have also been investigated by Kane et al.[51] and Müller et al.[52]. Multiquantum-well samples with $L_{w}=70-80 \AA$ gave intersubband absorptions near $\lambda_{\mathrm{p}}=9.3 \mu \mathrm{m}$ and $L_{w}=100 \AA$. resulted in a shift to a longer wavelength of $\lambda=11 \mu \mathrm{m}$. These measurements are in agreement with their calculated oscillator strengths and energy levels. 


\subsection{Asymmetric Quantum Well}

For a simple rectangular QW, we can only vary the well width and the barrier height (composition). In addition, the dipole matrix elements of transitions for odd-to-odd or even-to-even (i.e., 1-3 or 2-4) quantum numbers disappear (meaning these kind of transitions are not allowed) since the envelope functions of these energy states have the same parity due to symmetry of the well. However, the symmetry of the well can be broken by applying a strong electric field [53] or doping the QW heavily [54]. A high bias can produce a large leakage current, which will degrade the performance of the device while for the heavily doped case, the large thermionic emission associated with the high doping densities will prevent operation at higher temperatures. An alternative approach to achieving normal incidence operation is to use an asymmetric structures, which can be formed in different ways as shown in Figure:2.8.

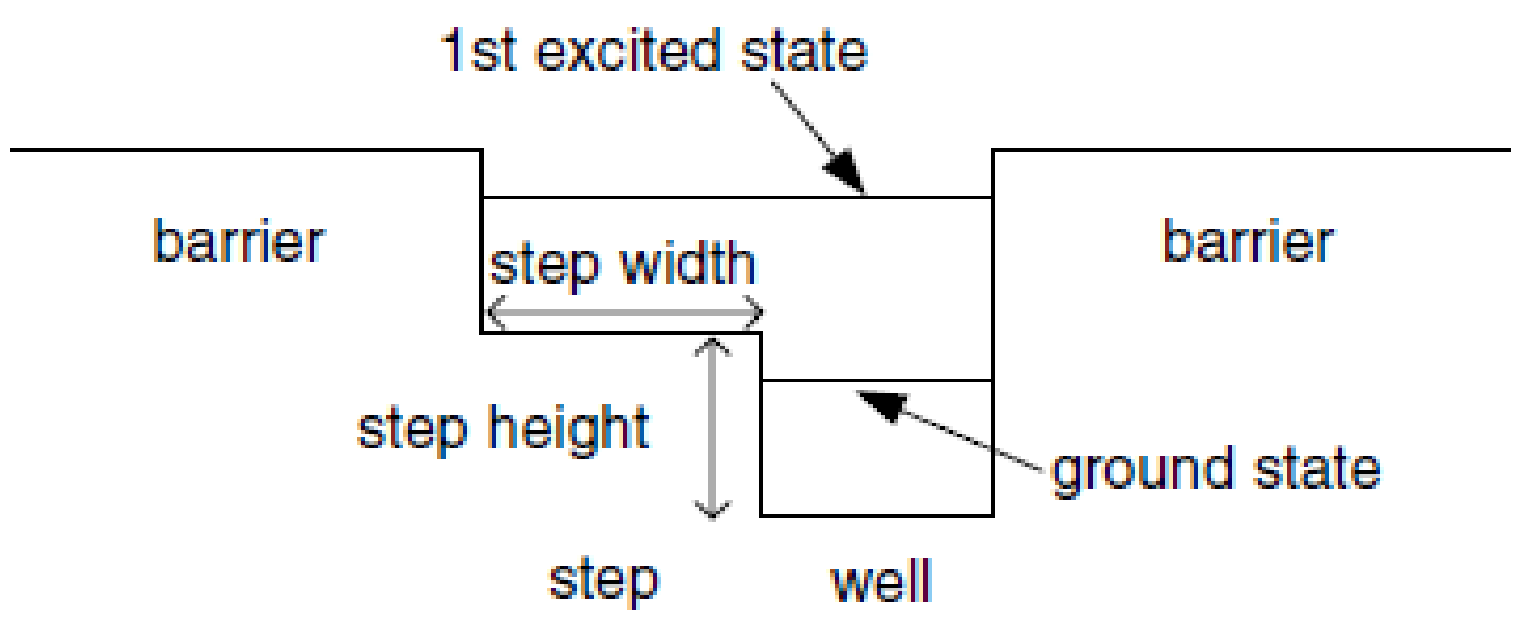

Figure: 2.8 Asymmetric QW structures: graded dope and stepped wells 
The introduction of the step inside the well results in normally forbidden transitions becoming allowed. Furthermore, the transition energies and related oscillator strengths can now be tuned "independently" by changing the well width, the step width, and the step height. This gives an advantage over the high electric field and doping induced asymmetry in a simple square well [54-56]. A component of normal incidence absorption was observed in a stepped DBQW using InGaAs/GaAs QWs with AlAs/AlGaAs barriers that operates at $3 \mu \mathrm{m}$.

In simple rectangular wells only the well width $L_{w}$ and barrier height can be varied. By introducing an additional barrier inside the well (thereby creating two closely coupled wells), additional design freedom to adjust the energy levels and oscillation strengths is achieved. The intersubband absorption in such structures has been studied both theoretically and experimentally [53-55]. Trzeciakowski and McCombe [56] show that a thin (e.g., 4 monolayer) barrier of AlAs in the center of a GaAs well can shift the ground state upward by $\sim 50 \mathrm{meV}$ while hardly affecting the excited state. This strong difference is a result of the groundstate wave function having a maximum in the center of the well (where the extra barrier is) while the excited state has a minimum. Intersubband absorption experiments and theoretical analyses by Lorke et al [57] confirm this large decrease in $\left(E_{2}-E_{l}\right)$ by a thin AlAs barrier in the quantum well. In a closely related study by Helgesen et al.,[58] the intersubband absorption energy and dipole matrix element of two closely coupled wells were calculated and compared with experiment. They find that

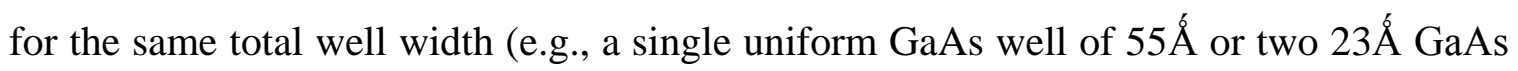
wells coupled by a $9 \AA$ barrier) the double-well structure has a significant enhancement of the dipole matrix element, due to the double maximum shape of the ground-state wave 
function. However, this coupled well structure also has a significantly smaller intersubband transition energy. Interestingly, if the width and barrier height of the double-well structure are adjusted so that the bound-to-bound transition occurs at the same energy as that of the usual single rectangular well, the dipole matrix elements of the two structures are essentially identical. Another method of tailoring the energy levels in a quantum well is by the use of $\delta$ doping. Temple et al.[59] have shown that the $\delta$ doping can itself generate a shallow quantum well in uniform material with transitions in the far infrared $(\lambda \sim 100 \mu \mathrm{m})$.

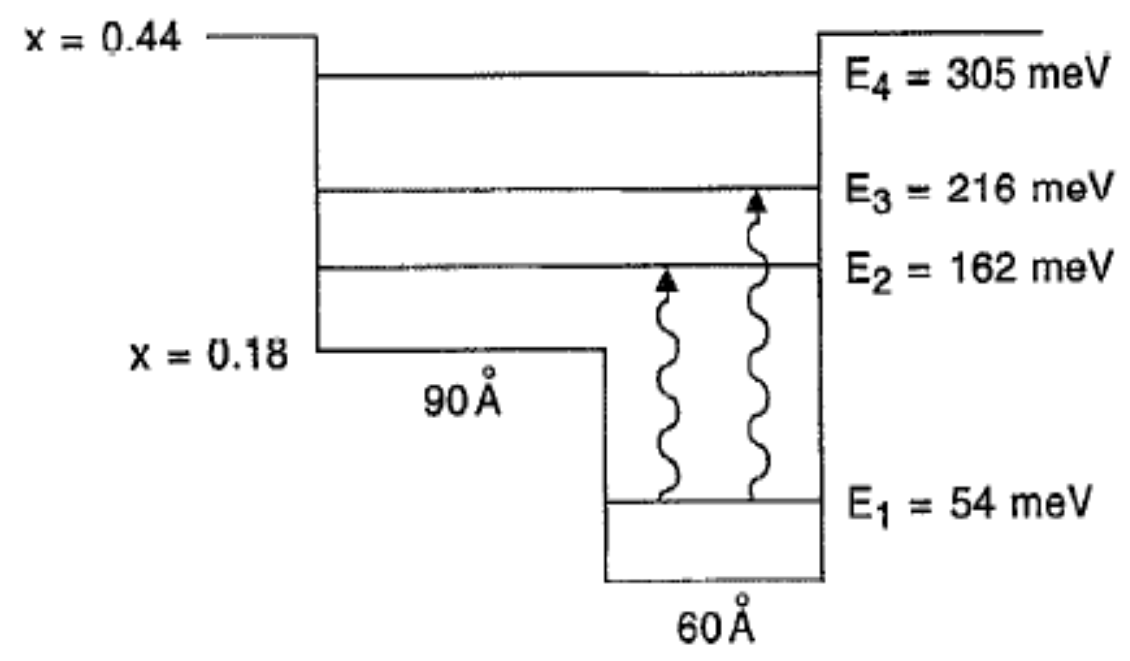

Figure: 2.9. Stepped quantum well indicating that the $E_{1}$ to $E_{2}$ transition and the $E_{1}$ to $E_{3}$ transitions are both allowed.

In symmetric quantum wells only transitions that change parity [i.e., between even and odd states so that $\Delta j=$ odd in are allowed; this restriction is removed in asymmetric wells. This a point of discussion which has been thoroughly covered in chapter 3. By doping only one side of the well, it is shown that this doping asymmetry 
allows the observation of a large number of intersubband transitions which are forbidden in uniformly doped wells. By using a stepped quantum well (as shown in Figure: 2.9) much stronger asymmetries can be introduced, [56] increasing the freedom to control the positions of the energy levels and oscillator strengths. Most important, by spatially separating the wave-function maxima of the ground and excited states strong electricfield effects can be obtained (as discussed in the following subsection). It has been investigated that for the structure shown in Figure. 2.9 the experimental oscillator strength of the (usually forbidden) $1 \rightarrow 3$ transition (at $h v=150 \mathrm{meV}$ ) was $65 \%$ that of the normally allowed $1 \rightarrow 2$ transition (at $112 \mathrm{meV}$ ) [60]

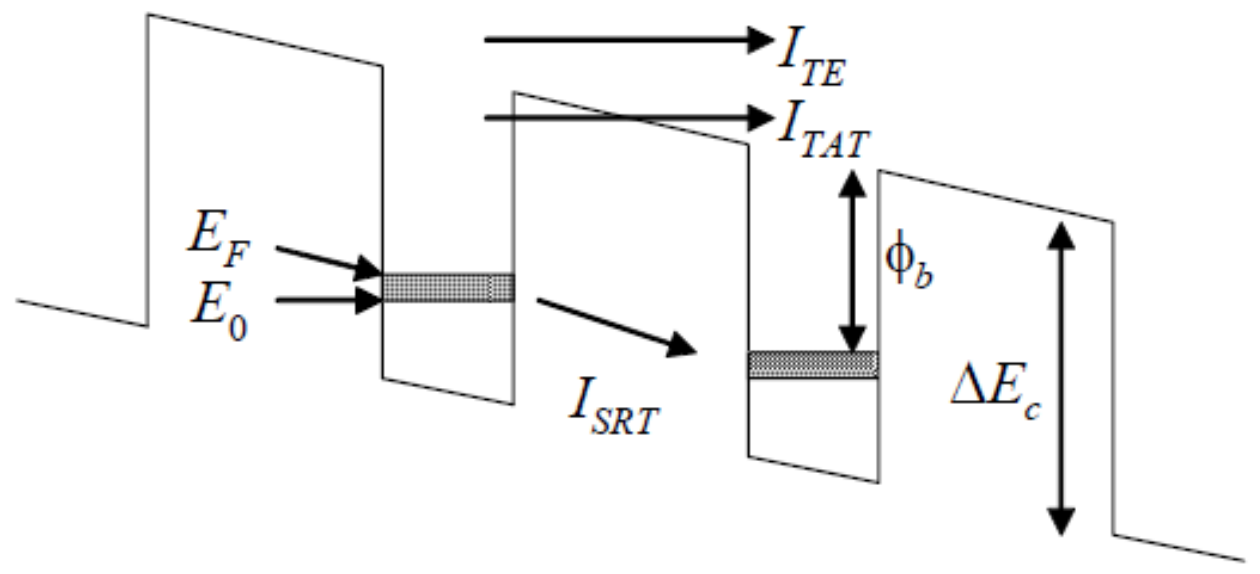

Figure: 2.10 Dark current transport in a typical QWIP. The three mechanisms shown are sequential resonant tunneling (ISRT), thermionic emission (ITE) and thermally assisted tunneling (ITAT). At low bias, $\phi_{b}=\Delta E_{c}-E_{0}-E_{F}$ is the barrier height seen by electrons in the ground state $E_{0}$ of the QWs. $\Delta E_{c}$ is the conduction band offset and $E_{F}$ the Fermi energy measured from $E_{0}$. 


\subsection{Dark current}

\section{Dark current and activation energy}

Dark current is the current that flows through a photodetector in the absence of incident radiation. Dark current in most QWIPs is caused by sequential resonant tunneling at low temperatures (typically $4-40 \mathrm{~K}$ ), and thermionic emission (TE) and thermally assisted tunneling (TAT) at higher temperatures (typically 40-150 K) [2, 21]. These transport mechanisms are schematically indicated in Figure: 2.10. Thermally assisted impurity tunneling through defect states in the barrier is dominant in some QWIPs in this temperature range [2,23]. At even higher temperatures (usually $T>150$ $\mathrm{K})$, the detectors act like resistors with linear current-voltage (I-V) characteristics. TE and TAT currents exhibit an activated temperature dependence that is used to deduce the activation energy. The deduced values of activation energy can then be compared with simple dark current transport models to determine the quality and accuracy of wafer growth.

\subsubsection{Sequential resonant tunneling}

In order to treat QWIPs based on this absorption mechanism we now need to consider the application of electric fields to a multiquantum-well structure, and the tunneling escape and subsequent transport of these photoexcited electrons.

The first QWIP was demonstrated by Levine et al.,[9] and that was based on quantum wells containing two bound states. The infrared absorption due to the intersubband transition from the bound ground state to the bound excited state is followed by the photoexcited electrons tunneling out of the well, as shown in Figure. 2.12. 
These photocarriers, which escape from the well, are transported by the electric field in the continuum above the barriers for an excited-state lifetime $\tau_{L}$ during which they travel a distance $L$ (which is the mean free path for recapture back into the quantum wells) and thereby produce a photocurrent.[10]

In principle, due to the two-dimensional (2D) nature of the electron gas in the well, resonant tunneling is possible only when the energy levels in each well allign, a condition generally not fulfilled in the presence of an applied electric field. However, it is shown that in the presence of acoustic phonons and impurity scattering within each well, conservation of energy and momentum is relaxed and resonant tunneling is possible provided that $e V_{p}<<\hbar / \tau_{1}$, where $V_{p}$ is the potential difference per period between the adjacent wells and $\tau_{1}$ is the ground-state scattering time [10]. Therefore, at small bias the electrons are able to conduct by ground-state resonant tunneling through the ground states of each well and this has been schematically represented in Figure. 2.12(b). The first negative differential conductance peak thus indicates the disruption of the resonant tunneling.

Thus, for an applied bias low enough such that the ground-state resonant tunneling condition is still satisfied, the total current $I$ can be written as $I=I_{s t}+I_{t h}+I_{p t}$, where $I_{s t}$ is the sequential resonant tunneling contribution, $I_{t h}$ is due to thermionic emission, and $I_{p t}$ is phonon assisted tunneling, which is expected to be small. The first two contributions can be written as [10] 
(a)

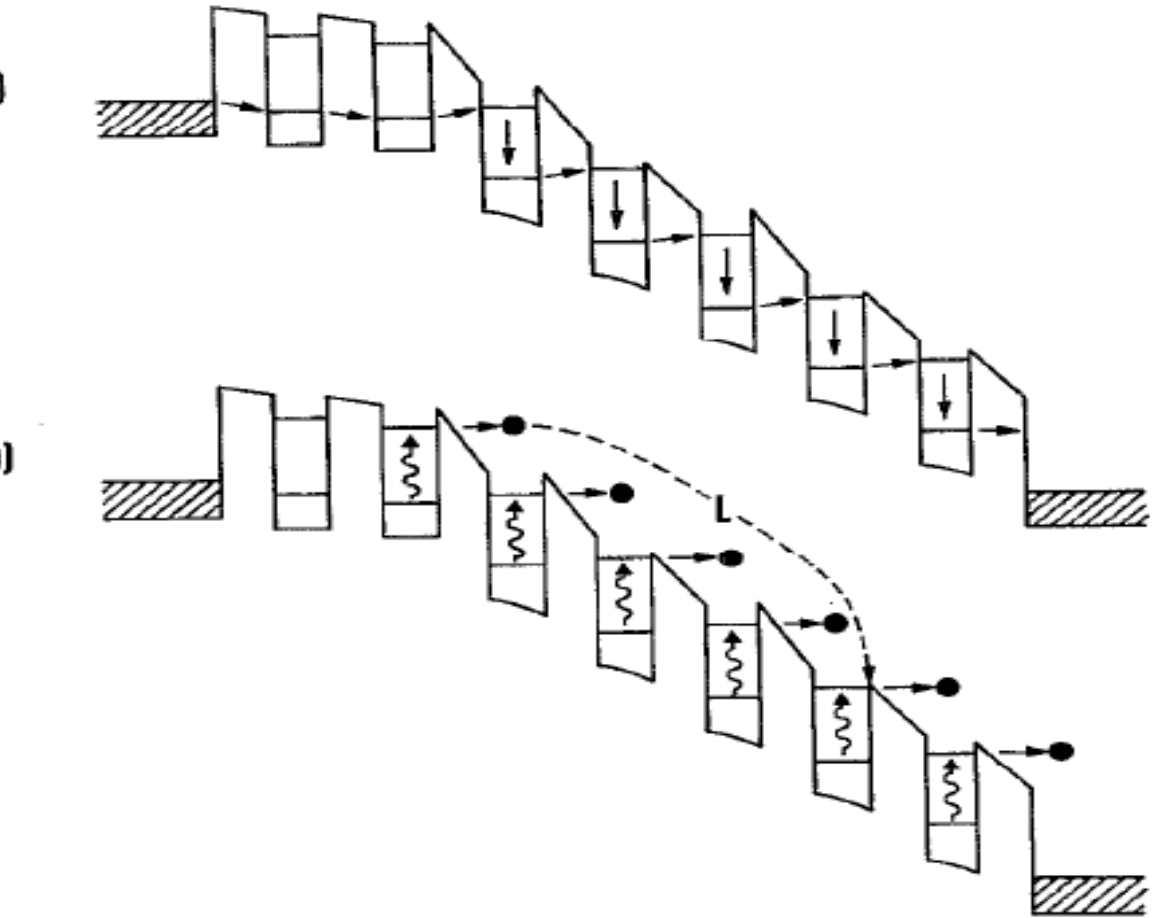

(b)

Figure: 2.11 (a) Sequential resonant tunneling in high-field domain (right-hand side); tunneling through ground state (left-hand side). (b) Photoconductivity produced by absorption of intersubband radiation followed by tunneling out of well [10].

$$
I_{s t}=\frac{e A}{\hbar L_{w}^{2}} T\left(V_{p}\right) k T \ln \left(\frac{1+e^{E_{F} / k T}}{1+e^{\left(E_{F}-e \Delta_{1}\right) / k T}}\right)
$$

and

$$
I_{s t}=\frac{e^{2} m^{*}}{\pi \hbar^{2}} \frac{A v_{D}}{L_{w}} \Delta_{1} e^{-\left(E_{b}-e \Delta_{2}-E_{F}-E_{1}\right) k T}
$$




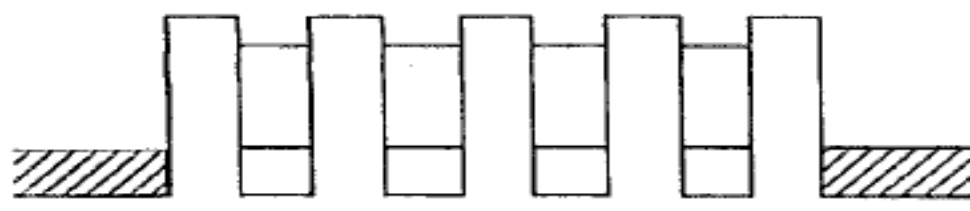

(a)

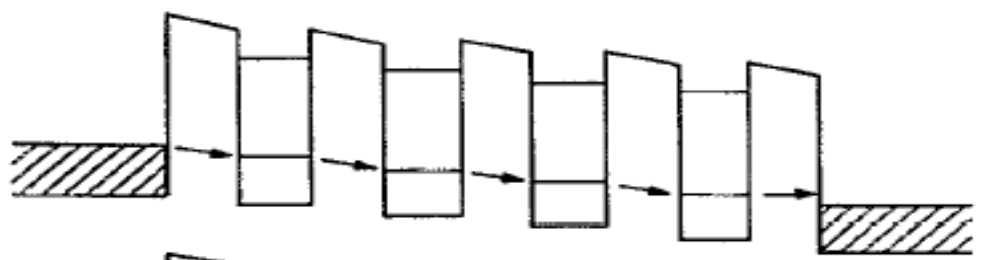

(b)

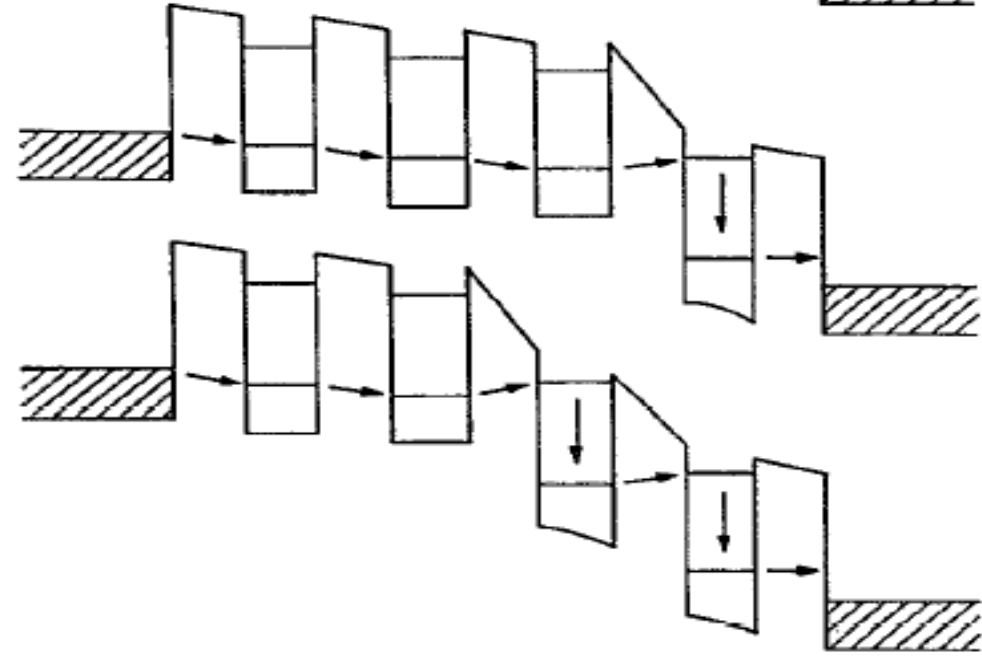

(c)

(d)

Figure: 2.12. Schematic band diagram of the superlattice for several values of the average potential drop per period $V_{P}$ (a) Zero bias. (b) Sequential resonant tunneling through the ground state $E_{1}$ for $V_{p}<2 \hbar / \tau_{1}$; arrows indicate electron transport. (c) Formation of first high-field domain for $V_{P}$ slightly greater than $2 \hbar / \tau_{1}$. Sequential resonant tunneling occurs through $E_{1}$ in the low-field region and through the first excited state $E_{2}$ in the high-field region. (d) Expansion of the high-field domain by one additional quantum well for a voltage increase of $\Delta V[10]$.

In the above equations, $A$ is the area of the device, $\Delta_{1}$ is the potential drop across one barrier, $E_{F}$ is the $T$-dependent Fermi energy, $\mathrm{m}^{*}$ is the effective mass of GaAs, $v_{D}$ is 
the effective drift velocity over the barrier, $E_{b}$ is the barrier height, and $\Delta_{2}$ is the potential drop across one well. In the WKB approximation, the barrier transmission coefficient is given by

$$
T\left(V_{p}\right)=\exp \left(\frac{-4 L_{b}}{3 e \hbar \Delta_{1}}\left(2 m_{b}^{*}\right)^{1 / 2}\left[\left(E_{b}-e \Delta_{2}-E_{2}\right)^{3 / 2}-\left(E_{b}-e V_{p}-E_{1}\right)^{3 / 2}\right]\right),
$$

where $m_{b}^{*}$ is the effective mass in the barrier, $L_{b}$ is the barrier thickness, and $V_{p}=\Delta_{1}+\Delta_{2}$ is the potential drop per period.

At high bias, i.e., when the potential drop $V p$ across a period is larger than $2 \hbar / \tau_{1}$, ground-state resonant tunneling is not possible and as a result negative differential resistance occurs. As each period breaks off from the resonant condition, the resistance across this period becomes much larger and a high-field domain forms Any subsequent increase in the bias will appear across this domain until the ground level rises to within $2 \hbar / \tau_{2}$ (where $\tau_{2}$ is the excited-state lifetime) of the first excited level $\mathrm{E}_{2}$ in the next well whereupon the resonant tunneling condition is restored [Figure: 2.12(c)]. Further increases in bias will cause another well to break off from the resonant condition and the I-V characteristic repeats [Figure. 2.12(d)]. Due to the screening effect of the spacecharge buildup at large bias and the consequent band bending, the domain formation is not a random process but occurs first at the anode and then extends one by one toward the cathode. As a result, one would expect there to be $p$ - 1 negative conductance peaks for a device with $p$ periods. Indeed in Figure. 2.13, 48 oscillations can be identified, [10] in agreement with this interpretation. 


\subsubsection{Reduction of dark current:}

The intersubband absorption excites an electron from the doped ground state to the second bound state where it can tunnel out, thereby producing a photocurrent. For this two-bound state structure, the absorption in the continuum above the top of the barriers is very weak, since most of the oscillator strength is concentrated in the bound-to-bound state transition. However, by decreasing the size of the quantum well, the strong oscillator strength of the excited bound state can be pushed up into the continuum resulting in a strong bound-to-continuum state absorption. [13] This extended state structure has the major advantage that the photoexcited electron can escape from the quantum well without tunneling through the energy barrier (as indicated in Figure. 2.13).

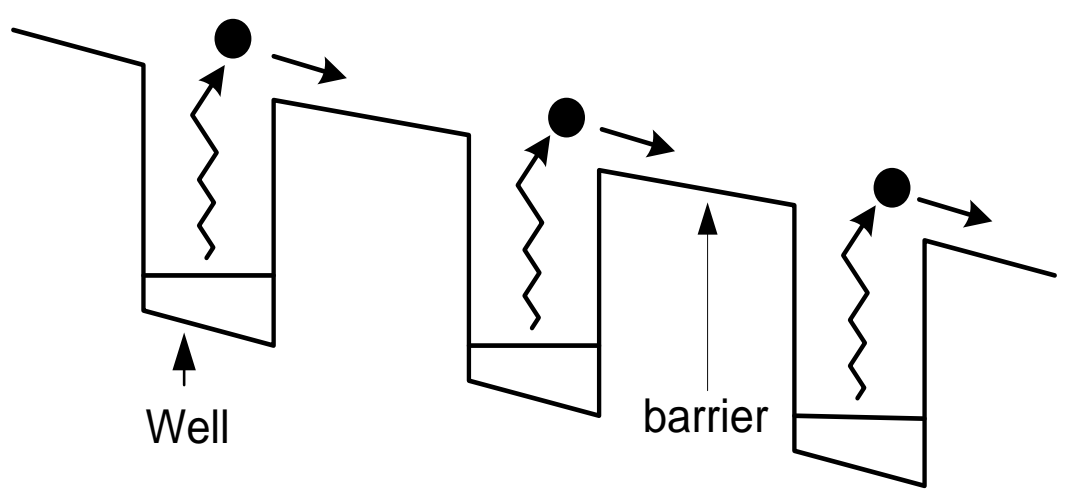

Figure: 2.13 A QWIP structure which employs bound-to-continuum

Thus, the bias voltage required for the photoelectron to efficiently escape from the well can be dramatically reduced, strongly lowering the dark current. In addition, the barrier thickness can now be substantially increased thereby further reducing the ground-state sequential tunneling by many orders of magnitude. By making use of these 
improvements, Levine et al. [15] demonstrated the first bound-to-continuum QWIP and achieved a dramatic increase in the performance, i.e., detectivity, by several orders of magnitude. This is experimentally confirmed by results of dark current versus temperature and bias for two different samples.[15] In order to understand this dramatic dark current reduction, we first calculate the effective number of electrons $n^{*}(V)$ which are thermally excited out of the well into the continuum transport states, as a function of bias voltage $V$. This is given by [I7]

$$
n^{*}(V)=\left(\frac{m^{*}}{\pi \hbar^{2} L_{p}}\right) \int_{E_{1}}^{\infty} f(E) T(E, V) d E,
$$

where the first factor containing the effective mass $\mathrm{m}^{*}$ is obtained by dividing the twodimensional density of states by the superlattice period $L_{p}$ (to convert it into an average three-dimensional density), and where $f(E)$ is the Fermi factor

$$
f(E)=\left[1+\exp \left(E-E_{1}-E_{F}\right) / k T\right]^{-1}
$$

$E_{1}$ is the bound ground-state energy, $E_{F}$ is the two dimensional Fermi level (measured relative to $\left.E_{1}\right)$, and $T(E, V)$ is the bias-dependent tunneling current transmission factor for a single barrier. We have used the total energy $E$ since for a real system, electron scattering causes the electron wave function to decay in the barriers according to $E$ rather than $E_{1}$ (which would be appropriate in ideal case). Equation (2.11) accounts for both thermionic emission above the energy barrier $E_{b}$ (for $E>E_{b}$ ) and thermionically assisted tunneling (for $E<E_{b}$ ). We can now calculate[17] the bias-dependent dark current $I_{d}(V)$, using $I_{d}(V)=\mathrm{n}^{*}(V) e v(V) A$, 
where $e$ is the electronic charge, $A$ is the device area, and $v$ is the average transport velocity given by $v=\mu F\left[1+\left(\mu F / v_{s}\right)^{2}\right]^{-1 / 2}$, where $\mu$ is the mobility, $F$ is the average field, and $v_{s}$ is the saturated drift velocity.

A novel alternative approach is to use thick (around 500 Á) barriers for strongly reducing the ground-state sequential tunneling. This has been discussed by Choi et al. [24]. Their proposed three-terminal structure (indicated schematically in Figure. 2.14) consists of thin $150 \AA$ barriers in the multiquantum well section of the QWIP, followed by a thick $1000 \AA$ collector barrier which acts as a high-pass filter for the photocurrent and a blocking contact for the ground-state tunneling dark current. By independently biasing the emitter and collector contacts a new hot-electron spectroscopy, which can determine the electron energy distribution, has been demonstrated

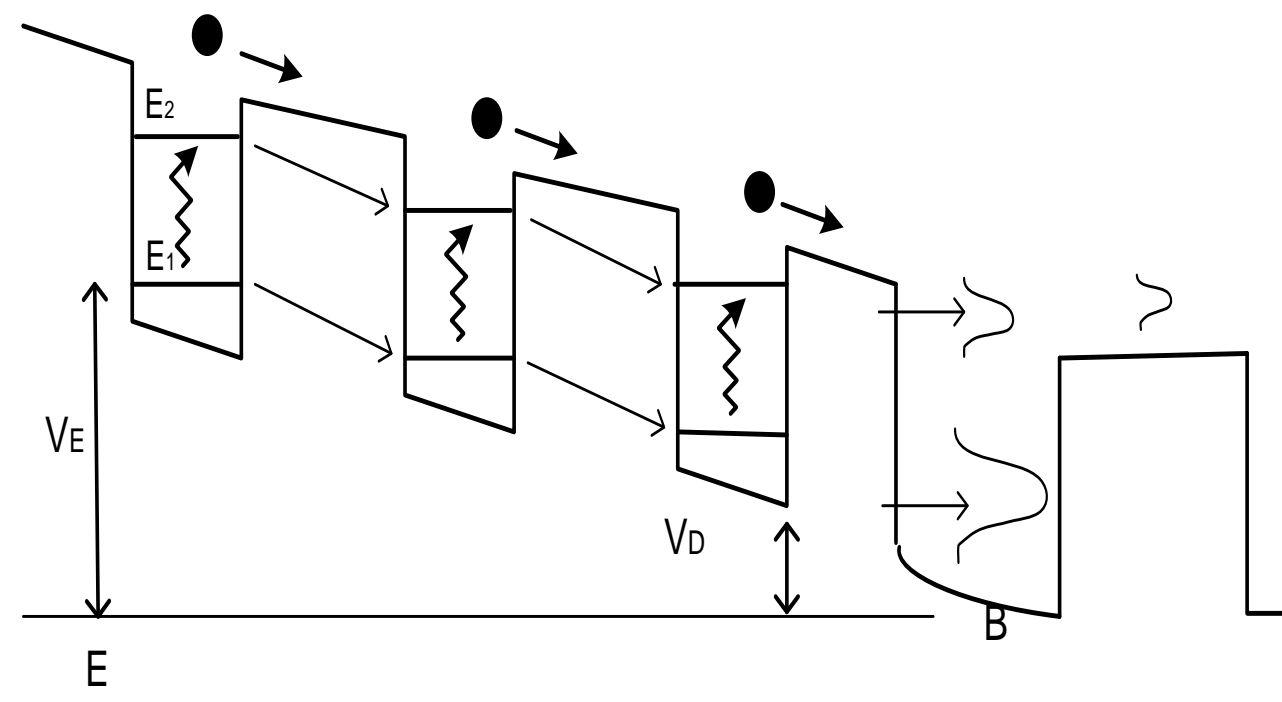

Figure 2.14. Conduction-band structure of a biased three terminal QWIP(emitter E, base B, and collector C). The collector barrier blocks the tunneling current from the ground state $E_{1}$. 
It has been found that the energy distribution of the dark current electrons (which includes tunneling through the barriers) is lower than that of the photoexcited carriers even after traveling through many (e.g., 50) periods. Thus, by optimally biasing the collector voltage to adjust the hot-carrier analyzer bandpass energy, the dark current can be preferentially reduced relative to the photocurrent. Even though this approach may lower the detectivity $\mathrm{D}^{*}$ (see Sec. V K), for situations where the dark current is so large that it saturates the imaging array multiplexer capacitors, the noise-equivalent temperature difference NE $\Delta \mathrm{T}$ (see Sec.XVII) can actually be improved since longer integration times can be used (this is discussed further in Sec. XVII). Although these three-terminal QWIPs are more complicated to fabricate, they allow more flexibility in the biasing and imaging array multiplexer design.

\subsubsection{Temperature dependence of the dark current}

It has been shown that Eq. (2.11) gives a very good account of both the temperature as well as the bias-voltage dependence of the dark current. A much simpler expression which is a useful low-bias approximation can be obtained by setting

$T(E)=0$ for $E<E_{b}$ and $\mathrm{T}(E)=1$ for $E>E_{b}$, resulting in

$n^{*}=\left(m^{*} k T / \pi \hbar^{2} L_{p}\right) e^{-\left(E_{c}-E_{F}\right) / k T}$,

where we have set the spectral cutoff energy $E_{c}=E_{b^{-}} E_{l}$. Therefore,

$$
I_{d} / T \propto e^{-\left(E_{c}-E_{F}\right) / k T},
$$

where the Fermi energy can be obtained from

$$
N_{D}=n_{0} \ln \left(1+e^{E_{F} / k T}\right)
$$


and

$n_{0} \equiv\left(m^{*} k T / \pi \hbar^{2} L_{w}\right)$

Thus, by plotting the dark current as a function of temperature we can obtain $\left(E_{c}-E_{F}\right)$ and compare it with the spectral cutoff energy $E_{c}$ measured optically. Similarly, by plotting $\ln \left(I_{d}\right)$ vs $\left(E_{c}-E_{F}\right)$ we should obtain a slope of $(-1 / k \mathrm{~T})$.

\subsection{Structure and Material Consideration}

QWIPs were originally built on $\mathrm{GaAs} / \mathrm{Al}_{x} \mathrm{Ga}_{1-\mathrm{x}} \mathrm{As}$ lattice-matched multiple quantum wells [6]. By varying the $\mathrm{Al}$ molar ratio $x$ in the barriers, a wide range of intersubband absorption wavelengths can be obtained. The intersubband transitions in modulation doped GaAs/AlGaAs

quantum wells was first demonstrated by West and Eglash [22] and later in directly doped wells by Levine et. al. [24]. The large oscillator strengths of these intra-band transitions, together with the flexibility to engineer the materials band gaps through composition choice, opened up the possibilities of different applications in nonlinear optics, electro-optic modulation and infrared detection. The idea of using such transitions in infrared detection was first suggested by Chui et al.[60] and later it was refined by and pursued by Levine et al. [11].

The most important features of these QWIPs involving III-V semiconductors that make them superior to MCT based ones are the chemical stability of the materials involved and their well developed growth-process technology. QWIPs have become dominant in the area of long wavelength IR focal plane array (FPA) applications in the 
last few years in terms of array size, uniformity, yield and cost of the systems. The other properties of QWIP, such as high impedance, fast response time, long integration time and low power consumption are well tailored to the requirements for the fabrication of large FPAs.

As previously mentioned, the AlGaAs/GaAs multiple-quantum-well (MQW) detectors, because of it's lattice matched structure have been preferred by the investigators and hence have matured compared to other QWIPs. However, modulation speed is still an issue which needs to be resolved in these MQW detectors. Although the maximum frequency of infrared detection has been theoretically predicted to be $100 \mathrm{GHz}$ , experimental demonstration has been around $80 \mathrm{GHz}$ [9]. Since modulation or detection speed is a function of electron mobility and carrier lifetime, the proposed AlGaAs/ InGaAs/InGaAs/AlGaAs structure holds better promise in terms of detection speed, due to higher carrier velocity of InGaAs.

\subsubsection{Strain and Critical layer thickness}

The accommodation of misfit across the interface between an epitaxial layer and its substrate is considered in this research. If the misfit between a substrate and a growing epilayer is sufficiently small, the first few atomic layers will be strained to match the substrate. However, as the layer thickness increases the homogeneous strain energy becomes so large that a point is reached after which misfit dislocations are introduced. The maximum layer thickness achievable without any defect is called the critical layer thickness (CLT). This thickness is calculated for the InGaAs epilayer on GaAs substrate 
using three different approaches. The approaches for calculating the thickness are the following

- Investigating the conditions under which an existing threading (grown-in) dislocation in the film might bow and migrate under the influence of misfit stress to generate a misfit dislocation line [17]. The critical layer thickness in a grown-in threading dislocation approach is given by

$$
h_{c} \cong\left(\frac{b}{f}\right)\left[\frac{1}{4 \pi(1+v)}\right]\left[\ln \left(\frac{h_{c}}{b}\right)+1\right]
$$

where $\mathrm{b}$ is the slip distance $\mathrm{f}$ is the misfit and $v$ is Poisson's ratio.

- Investigation of the conditions that are expected to lead to the nucleation and expansion of halfloops. Van der Merwe[61] determined critical layer thickness by calculating the coincidence of the areal strain energy density in the film with the interfacial energy between the film and the substrate.

$$
h_{c} \cong\left(\frac{1}{8 \pi^{2}}\right)\left(\frac{1-v}{1+v}\right) \frac{a_{0}}{f}
$$

where $a_{0}$ is the bulk lattice constant of the substrate.

- According to R. People and J. C. Bean [62], critical layer thickness is calculated assuming that the growing film is initially free of threading dislocations and that interfacial misfit dislocation will be generated when the areal strain energy density exceeds the self-energy of an isolated dislocation.

The areal strain energy density associated with a film of thickness $h$ is given by

$$
\varepsilon_{H}=2 G\left(\frac{1+v}{1-v}\right) h f^{2}
$$


where $\mathrm{G}$ is the shear modulus and the critical layer thickness is given by:

$$
h_{c} \cong\left(\frac{1.9 \times 10^{-2} \mathrm{~A}}{f^{2}}\right) \ln \left(\frac{h_{c}}{4 \mathrm{~A}}\right)
$$

\subsection{Conclusion}

In this chapter the physical mechanism of infrared detection has been presented. Photons, having energy corresponding to infrared region, are absorbed by exciting electrons from the ground state to excited state of a quantum well, which is formed by sandwiching a low bandgap material in between two large bandgap materials. The absorption rate, which is quantified by absorption coupling coefficient, depends on the oscillator strength $(f)$ and the relative direction between dipole moment of displacement $(P)$ and polarization vector. The oscillator strength is actually nothing but the matrix element of the momentum operator. The reasoning behind choosing asymmetric quantum well structure has been presented. The InGAs/AlGaAs well system is superior as they promise improved detector responsivity achieved through higher electron mobility. On the other hand, exploitation of asymmetric (stepped) quantum well enables the absoption of normal incidence, which is very important for many applications requiring array operation. The chapter also presents a detailed discussion on dark current transport mechanism in the quantum well structures considered for infrared photodetection, along with the impact of temperature on the process. Finally, the effect of strain has been discussed. 


\section{CHAPTER 3}

\section{Quantifying Inter-subband Transition in Asymmetric Quantum Wells}

With the advancement of III-V semiconductors process technology, quantum well infrared photo-detectors (QWIPs) fabricated with such materials have become the most promising alternative to conventional $\mathrm{HgCdTe}$ based photo detection technology [1]. Understandably, optical absorption and electron transport mechanisms in QWIPs structures have generated considerable research interest [2-5]. Absorption of electromagnetic radiation in quantum well $(\mathrm{QW})$ structures can involve inter-subband transitions of electrons within the same band. Owing to the fact that, such transitions correspond to small energy differences among the bound quantum states and therefore, are particularly suitable for infrared detection.

Initially when first introduced, QWIPs were fabricated on multiple rectangular quantum wells (QWs) composed of lattice-matched III-V materials, such as GaAs/ $/ \mathrm{Al}_{\mathrm{x}} \mathrm{Ga}_{1-x} \mathrm{As} \mathrm{QW}$ structures. As can be seen from the band structure of GaAs (shown in Figure: 3.1 ) that forms the well region, electrons tends to occupy the $\Gamma$ valley where certain conditions for the symmetry of the wave function exist. As detailed later, symmetry conditions impose a "selection rule" that diminishes the probability of electron transition among certain eigen states having definite parity [6]. Moreover it also forbids transition being induced by photons with no electric field component in the growth 
direction. Consequentially, photons with transverse electric (TE) polarization with respect to the growth direction cannot be absorbed in rectangular QW structures [7-8]. To overcome such an eventuality, a number of ingenious techniques have been proposed, including fabrication of grating structures on top contact, illumination through $45^{\circ}$ facet, and a scheme to arrange multiple

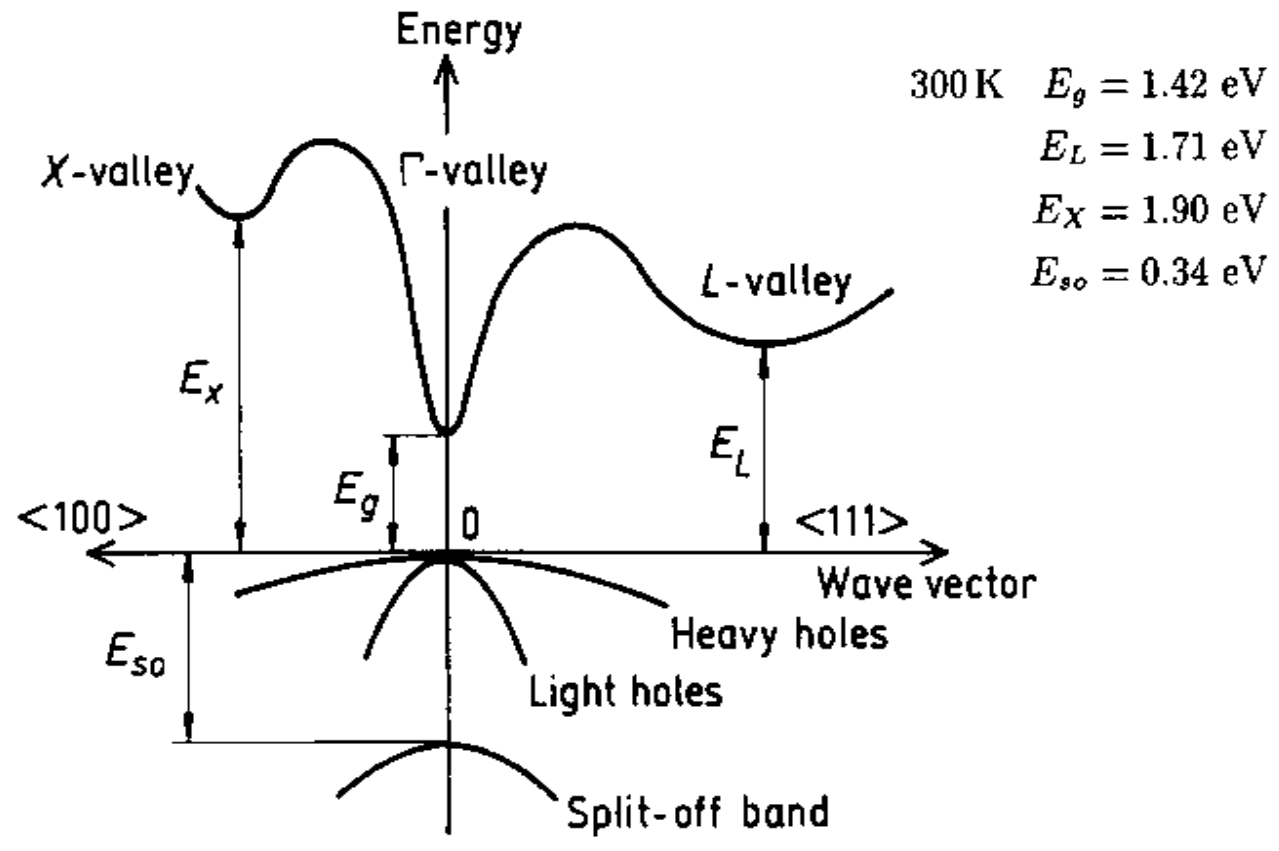

Figure: 3.1. Band structure of GaAs. $300 \mathrm{~K}$

pass of photons with oblique incidence[3], [9]. Unfortunately, all these alternatives not only complicate the focal plane fabrication process, but also induce cross talks among adjacent pixels [10].

Asymmetric QWs have been adopted to break the wave function symmetries, despite the fact that such symmetry conditions does not strictly hold for real rectangular 
wells due to various reasons like surface roughness, truncation of periodic bulk structure, etc. Achievable through steps in quantum well, asymmetry "distorts" the bound state wave functions in a way that they no longer posses' definite parity [10]. Investigation on inter-subband transition in QWIPs with asymmetric structures have been based on both single band and eight(ten)-band k.p model, the later being combined with envelope function Fourier expansion [11].

In this chapter the mesoscopic representation of the quantum processes that govern both vertical (absorption) and parallel (tunneling) electron transport in asymmetric QW structures are utilized to quantify QWIP performance parameters. Due to the scattering potential felt by electrons in the presence of photons, calculation of wavefunctions only at eigen energies becomes inadequate. The work presented here, therefore, determines the density of states (DOS) as a function of energy using nonequilibrium Green's function(NEGF) formalism and quantum mechanical wave impedance concept [12]. This facilitates calculation of broadening of states resulting from both photon absorption and tunneling. Such broadening, instead of being calculated, had been simply assumed in previously reported works [11]. The chapter is organized as follows: first the formulation for calculating absorption rate is presented, followed by a discussion on selection rule and definition of dipole matrix element, which quantifies the strength of coupling among different eigen states. The next section presents a comprehensive analysis of an $\mathrm{AlGaAs} / \mathrm{GaAs} / \mathrm{InGaAs}$ asymmetric quantum well structure showing how asymmetry introduced through stepped quantum well can improve photon absorption at infrared region. 


\subsection{Quantifying Absorption Rate}

The transition rate or probability of transition per unit time from one energy eigenstate of a quantum system into a continuum or other eigenstates due to any perturbation can be calculated by Fermi's Golden Rule. As an obvious consequence of Heisenberg's Uncertainty principle of energy and lifetime, energy of a quantum state smears out or 'broadens' as particles leaks out of that state. Broadening of an energy state $i$ due to electron transition to another state, $f$, therefore can be calculated by Fermi's Golden rule [13]:

$$
\Gamma_{i}(\hbar \omega)=2 \pi\left|\rho_{i f}\right|^{2} D_{f}(\hbar \omega)
$$

where $\rho_{i f}$ is the coupling constant between the states $\psi_{i, f}, D_{f}$ is the DOS of the final state and $\hbar$ is the reduced Planck's constant and $\omega$ is the angular frequency. The absorption coupling constant can be evaluated simply from the matrix elements of the scattering potential $\mathrm{U}^{\mathrm{ab}}$

$$
\rho_{i f}^{a b}=\int \psi_{i}^{*}(r) U_{i f}^{a b} \psi f(r) d r \equiv\left\langle f\left|U_{i f}^{a b}\right| i\right\rangle
$$

Where $\psi_{i}$ and $\psi_{f}$ are the wave functions of states $i$ and $f$ respectively. The entire problem of calculating $\rho_{\text {if }}$ amounts to finding the scattering potential $U_{s}$ that an electron feels in presence of a photon with particular frequency:

$$
U_{s}(r, t)=U^{a b}(r) \exp (-j \omega t)
$$

Once the interaction potential is identified, the absorption coupling constant can be evaluated simply with eqn. 3.2. However, the calculation of the matrix elements becomes somewhat complicated due to the fact that the effect of electromagnetic field associated with a photon enters the Schrödinger equation through the vector potential rather than the 
scalar potential. We first write down the electric field due to a single photon in mode $(\beta, v)$ in the form:

$$
\vec{E}=\hat{v} E_{O} \sin [\vec{\beta} \cdot \vec{r}-\omega(\beta) t]
$$

where amplitude $E_{O}$ is calculated by equating the associated energy to that of the photon and assuming $V$ to be the volume and $\varepsilon$ to be the permitivity:

$$
\varepsilon E_{O}^{2} V / 2=\hbar \omega \rightarrow\left|E_{O}\right|=\sqrt{\frac{2 \hbar \omega}{\varepsilon V}}
$$

Using the fact that for electromagnetic wave the electric field $E$ is related to the vector

$$
\begin{aligned}
\text { potential } A \text { as } \vec{E}=-\partial \vec{A} / \partial t & \\
\vec{A}(\vec{r}, t) & =\hat{v} A_{O} \sin [\vec{\beta} \cdot \vec{r}-\omega(\beta) t] \text { with }\left|A_{O}\right|=\sqrt{\frac{2 \hbar}{\omega \varepsilon V}}
\end{aligned}
$$

Defining the absorption portion (the other portion being the emission) of the vector potential due to photon as $A^{a b}(r)=\hat{v}\left(A_{O} / 2\right) \exp (-j \omega t)$, the coupling coefficient for the absorption process can be written as:

$$
\rho_{i f}^{a b}(\vec{\beta}, \hat{v})=\left(e A_{O} / 2\right)\langle i|\exp (-j \vec{\beta} \cdot \vec{r}) \vec{p} \cdot \hat{v}| f\rangle
$$

Where $\vec{p}=-j \hbar \vec{\nabla} \equiv-j \hbar \partial / \partial z$, assuming a one-dimensional case with $z$ being the propagation direction. In case of QWIP, wavefunctions are localized to a dimension shorter than the optical wavelength under consideration, and therefore $\exp (j \vec{\beta} \cdot \vec{r})$ can be neglected. So eqn. (3.7) becomes:

$$
\rho_{i f}^{a b}(\vec{\beta}, \hat{v})=\left(e A_{O} / 2\right)\langle f|\vec{p} \cdot \hat{v}| i\rangle=\frac{e}{m^{*}} \sqrt{\frac{\hbar}{2 \varepsilon \omega V}}(P \cos \theta)
$$


Here $P=\langle f|\vec{p}| i\rangle$ is the dipole moment of the transition and $\theta$ is the angle between $P$ and the electric field polarization $\hat{v}$. It is worth mentioning here that for a specific combination of given quantum well and light polarization, $P \cos \theta$ can be zero and therefore light with that polarization will not be absorbed. This fact forms the basis of the so-called selection rule, which has been discussed later.

The transition probability is $T_{i \rightarrow f}=\Gamma_{i} / \hbar$, which is an obvious consequence of Heisenberg's uncertainty principle. The net transition rate from $i$ to $f$ is:

$\phi_{i f}(\hbar \omega)=\frac{2 \pi}{\hbar}\left|\rho_{i f}\right|^{2} D_{f}(\hbar \omega)\left[f_{i}(\hbar \omega)-f_{f}(\hbar \omega)\right]$

where $f_{i, f}$ are the Fermi-Dirac distribution of electrons in respective sub-bands. By defining DOS in terms of broadening, the absorption co-efficient $\left(\alpha_{i \rightarrow f}\right)$ between $i$ and $f$ can now be written as [11]:

$\alpha_{n m}(\hbar \omega)=\frac{2 \pi q^{2}}{n_{r} c \varepsilon_{o} m_{o}^{2} \omega V}\left|\rho_{i f}\right|^{2}\left(f_{i}-f_{f}\right) \times \frac{(\Gamma / 2 \pi)}{\left[E^{\prime 2}+(\Gamma / 2)^{2}\right]}$

Where $n_{r}$ is the refractive index, $\varepsilon_{o}$ is the free space dielectric constant, $c$ is the speed of light, $V$ is the volume and $E^{\prime}=\left(E_{f}-E_{i}-\hbar \omega\right)$.

The transition momentum matrix element or coupling constant $\rho_{\text {if }}$ is also used to define a performance parameter, dipole oscillator strength $f(\hbar \omega)$, which is directly proportional to the absorption coefficient. $f(\hbar \omega)$ is defined as:

$$
f(\hbar \omega)=\frac{2 \hbar}{m^{*} \omega}\left|\left\langle\psi_{f}\left|\frac{\partial}{\partial z}\right| \psi_{i}\right\rangle\right|^{2}
$$

where $\hbar \omega$ is the incoming photon energy, $m^{*}$ is the effective mass of the ground state, $\psi_{1}$ is the ground state wave function subjected to the normalization condition $\left\langle\psi_{i} \mid \psi_{i}\right\rangle=1$ 
within the quantum well structure, and $\psi_{f}$ is the normalized excited state wave function having an energy $\hbar \omega$ above the ground state.

\subsection{Impact of Effective Mass and Strain}

\subsubsection{Variation of effective mass with energy:}

Strictly speaking, in a material with spatially varying effective mass, the quantity $m^{*}$ in above equation and hence $f(\hbar \omega)$ are not well-defined. The absorption rate needs to be calculated directly from the Fermi golden rule, whose matrix element depends explicitly on $m_{n} *\left(E_{i}\right)$ of each layer. However, it turns out that for the material system studied in this work, $m_{n}{ }^{*}\left(E_{i}\right)$ in different layers are quite close to each other, e.g., being $0.063 \mathrm{mo}$ in the well region and $0.085 \mathrm{mo}$ in the barrier region for a given structure. Therefore it is useful in this work to treat $\psi_{1}$ to have an average effective mass and retain the concept of the oscillator strength. With this approximation, the quantity $m^{*}$ becomes a prefactor in Eq. (3.11), and its value will not affect the absorption spectral line shape. For simplicity, $m^{*}$ was chosen to be

$$
m^{*}=\frac{\sum_{n} m_{n}^{*} d_{n}}{\sum_{n} d_{n}}
$$

where $m_{n} *$ is the effective mass at the band edge of the material layer $n$. Besides the issue of $m^{*}$, if the full wave functions of the quantum well states are considered, Eq. (3.11) should also contain a multiplication factor $S \equiv\left|\left\langle\phi_{c}\left(E_{f}\right) \mid \phi_{c}\left(E_{i}\right)\right\rangle\right|^{2}$, where $\phi_{c}$ is the atomic wave function. However, the value of $S$ is typically very close to unity and varies very little across the spectrum. The presence of $S$ can thus be ignored. For example, using the 
certain structural parameters, the value of $S$ based on the two-band model can be shown to vary only slightly from 0.934 to 0.963 between $\lambda=2.9$ and $4.1 \mu \mathrm{m}$. The change of $S$ across the spectrum is therefore can be safely ignored.

\subsubsection{Impact of strain:}

The energy band gap $E_{g}$ of materials forming different layers of the quantum well structure determines the well depth and therefore dictates the absorption spectrum of any given structure. The strain developed due to juxtaposing different layers of material effect the bandgap of any given material. The unstrained band gap of the $n$th layer in the unit of $\mathrm{meV}$ is

$$
E_{g, n}^{u}=1424+1186 x_{n}+370 x_{n}^{2}-1499 y_{n}+429 y_{n}^{2},
$$

where $x_{n}$ and $y_{n}$ are the $\mathrm{Al}$ and In molar ratios in the $n$th layer, respectively. (In the present calculation, however, $x$ and $y$ cannot be both nonzero in a given layer, i.e., AlInGaAs quaternary compound is not considered). Since the material layers are under strain, the values of band gaps are modified. Assuming the strain is accommodated exclusively in the InGaAs well layers, which are usually much thinner than the GaAs/AlGaAs layers, the modified band gap for $\mathrm{y}_{\mathrm{n}}>0$ is

$$
\begin{aligned}
& E_{g, n}^{s}=E_{g, n}^{u}+2 a\left(1-\frac{c_{12}}{c_{11}}\right) \varepsilon_{11} \\
& \text { where } a=-8700+2700 y_{n} \mathrm{meV} \text { is the deformation potential, } \\
& c_{12} / c_{11}=\left(5.32-0.79 y_{n}\right) /\left(11.81-3.48 y_{n}\right) \text { is the ratio of the elastic constants, and } \\
& \varepsilon_{11}=-0.405 y_{n} /\left(5.66533-0.405 y_{n}\right) \text { is the hydrostatic component of the strain induced }
\end{aligned}
$$


by the adjacent GaAs/AlGaAs material layers. Therefore, in the present assumption, the band gap of InGaAs is widened while that of GaAs and AlGaAs are unchanged. The band offset $\Delta E_{n}$ is taken as

$$
\begin{aligned}
& \Delta E_{n}=0.60\left(E_{g, n}^{u}-1424\right) \quad \text { if } x_{n} \geq 0 \\
& =0.63\left(E_{g, n}^{u}-1424\right) \quad \text { if } y_{n}>0
\end{aligned}
$$

The effective mass at the conduction band edge is given by

$$
m_{n}^{*}=0.0665\left(1+1.256 x_{n}-0.579 y_{n}\right)
$$

Since these detectors involve high-energy states, band nonparabolicity has to be taken into account. The resulting effective mass $m_{n} *(E)$ can be expressed in the form of

$$
m_{n}^{*}(E)=m_{n}^{*}\left(1+\frac{E-\Delta E_{n}}{E_{g, n}}\right)
$$

where $E_{g, n}$ is the strain modified band gap. With all these material parameters, the input parameters, $m_{n}^{*}(E)$ and $k_{n}(E)$, can be obtained, and can be used to obtain eigen energy levels and state wave functions. With this information, $f(\hbar \omega)$ in Eq. (3.11) can be computed.

\subsection{Selection Rule and Normal Incidence}

For the intersubband transitions at the $\Gamma$-point in the conduction band of III-V materials, it is customary to assume that the transition in the QW would normally only be induced by light polarized along the growth direction of the QW. Initially this "selection rule" was thought to be supported by experimental observations made on earliest grown standard square QW-type structure. Over the time, it became an accepted fact that QW 
detectors cannot absorb light incident normal to the multi-QW interfaces in which the incident light has no polarization component along the growth direction. As a result various means of overcoming this difficulty were proposed and designed. One methodology was to exploit rear illumination achieved through a $45^{\circ}$ polished facet. However, it turned out that this arrangement is not suitable for two-dimensional infrared detector imaging arrays. Several alternatives were also devised. One of them was to etch a grating structure into the top contact layer of each pixel to scatter the incident photons into propagation paths at an angle to the multi-QW interfaces. Another alternative is to use a wave guide beneath the multi-QW mesas to allow the coupled radiation to enter at an angle and pass through the multi-QW stack several times. As a variation to this methodology, one could use a combination of these techniques, namely a grating coupled wave guide. The latter is formed by a reflection grating on one side of the multi-QW stack and a cladding layer on the other side. These grating-coupled wave guides can have quantum efficiencies of nearly unity for photons at normal incidence.

Unfortunately, despite being very ingenious in nature, such structures not only complicate the fabrication of focal plane arrays but they can also cause crosstalk between adjacent pixels. As a result there has been a continuing effort to design and optimize QW structures in which intersubband absorption of normally incident photon can be achieved. In the past such detectors have been fabricated using inter valence band transitions in SiGe and GaAs/AlGaAs [13] quantum wells. In case of SiGe structure, xy-polarized optical electric field can induce a transition for electronic motion along the growth (i.e., z) direction through the off-diagonal terms of the inverse mass tensor, whereas in case of GaAs/AlGaAs it is thought that the coupling between the Bloch states of the conduction 
and valence bands is responsible for the absorption. However, being $p$-type detectors, they have a responsivity which is typically an order of magnitude lower than that of $n$ type detectors. This is thought to occur as a result of the heavy mass and low mobility of holes in the valence band compared with that of electrons in the conduction band.

After the initial learning was achieved, several investigations have reported normal incidence intrasubband conduction-band absorption in various III-V QW structures. Similarly there have been accompanying theoretical articles aimed at explaining these observations. Although there is a degree of ambiguity with regard to the experimental observations (which has been discussed later) and a certain amount of controversy in relation to the precise theoretical explanation of the occurrence of normal incidence absorption, there is a growing body of evidence (both experimental and theoretical) that there is some doubt regarding the general validity of the selection rule forbidding normal incidence absorption in these material systems. Generally speaking, selection rules are a rigorous mathematical consequence of symmetry arguments applied to some well defined problem and, in consequence, the rule should be obeyed exactly. If evidence exists that sheds doubt on the validity of a proposed selection rule, then one of three possibilities arises: either the presumed symmetry does not exist, the problem is ill defined (i.e., it is not as well defined as was previously thought) or both assumptions are incorrect (i.e., the presumed symmetry is incorrect and the problem is ill defined). We will argue in the following, using concepts from pure quantum theory, that the latter of the three possibilities actually applies. An extension of these same arguments will be shown to lead to the conclusion that, for the most general QW structures (i.e., those with arbitrary shaped QW potential profiles) the selection rule will be broken and further that 
doping the QW structure in an appropriate manner can "relax" the selection rule even further.

For light polarized along the $z$ direction, i.e., the growth direction, the transition dipole moment matrix element or coupling coefficient between two given energy states $\psi_{1}$ and $\psi_{2}$ becomes, on using the envelope function approximation, $\left\langle\psi_{1}(x, y, z)\left|\frac{\partial}{\partial z}\right| \psi_{2}(x, y, z)\right\rangle$

$=a_{c}^{*} b_{c}\left[\left\langle\chi_{c}(x, y, z) \mid \chi_{c}^{\prime}(x, y, z)\right\rangle\left\langle U_{c}\left|\frac{\partial}{\partial z}\right| U_{c}\right\rangle+\right.$ $\chi c(x, y, z) \partial \partial z \chi c^{\prime}(x, y, z) U c U c$

$=a_{c}^{*} b_{c}\left[\left\langle\chi_{c}(z) \mid \chi_{c}^{\prime}(z)\right\rangle\left\langle U_{c}\left|\frac{\partial}{\partial z}\right| U_{c}\right\rangle+\right.$ $\chi c(z) \partial \partial z \chi c^{\prime}(z) U c U c$

Here $U_{c}$ is conduction-band Bloch function. It has been assumed that,

$$
\left|\psi_{1}(x, y, z)\right\rangle=a_{c} \chi_{c}(x, y, z)\left|U_{c}(x, y, z)\right\rangle
$$

i.e. we assume magnitude of $a_{c}$ squared is equal to 1 . Similarly for a second conduction subband state $\psi_{2}(\mathrm{z})$ we may write

$$
\left|\psi_{2}(x, y, z)\right\rangle=b_{c} \chi_{c}^{\prime}(x, y, z)\left|U_{c}(x, y, z)\right\rangle
$$

It is standard in the literature to assume that $\left\langle\chi_{c} \mid \chi_{c}^{\prime}\right\rangle=0$ (although, as we have pointed out above, there is no general requirement in quantum mechanics that this be true). To proceed we note that if, the periodic functions $\left|U_{c}(x, y, z)\right\rangle$ are chosen to be Bloch states corresponding to the bottom of the respective bands (i.e., to wave vectors $\mathbf{k}=0$ ) then, without loss of generality, they can be chosen to be real functions. For such a choice of Bloch function 


$$
\begin{gathered}
\left\langle U_{c}\left|\frac{\partial}{\partial z}\right| U_{c}\right\rangle \propto \int_{0}^{a} U_{c}(x, y, z) \frac{\partial}{\partial z}\left(U_{c}(x, y, z)\right) d z \\
=\left[\frac{1}{2} U_{c}^{2}\right]_{0}^{a}=0
\end{gathered}
$$

Thus, for conduction-band subband states for which the effective mass approximation holds (i.e., states close to the bottom of the band) we can assume that $\left\langle U_{c}\left|\frac{\partial}{\partial z}\right| U_{c}\right\rangle$ vanishes. Hence, with these approximations we obtain, since $\left\langle U_{c} \mid U_{c}\right\rangle=1$,

$$
\left\langle\psi_{1}(x, y, z)\left|\frac{\partial}{\partial z}\right| \psi_{2}(x, y, z)\right\rangle=a_{c}^{*} b_{c}\left\langle\chi_{c}(z)\left|\frac{\partial}{\partial z}\right| \chi_{c}^{\prime}(z)\right\rangle
$$

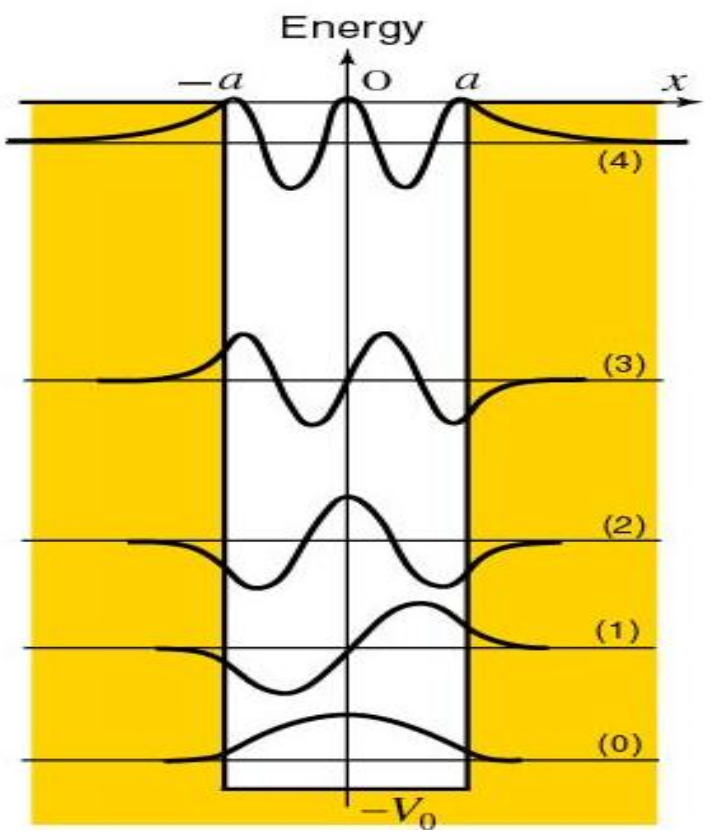

Figure: 3.2. Schematic representation of a perfect square $Q W$ and the wavefunctions of its bound states. The figures show the inversion symmetry of the wavefunctions. 
Since, for a general QW structure, there is no reason to expect that the integral on the rhs of Eq. (3.22) vanishes, we see that light polarized along the $z$ direction will be absorbed. As an aside, it is interesting to note that for a perfect, rectangular QW structure, the assumptions leading to Eq. (3.22) result in a"selection rule" even for light polarized along the $z$ direction. For a perfect rectangular QW the system possesses inversion symmetry and the eigenstates $\mid \psi_{1}(x)>$ and $\left|\psi_{1}(x)\right\rangle$ will have a definite parity, as shown Figure:3.2. Given that $\partial / \partial z$ has odd parity, it is clear that the matrix element on the rhs of Eq. (3.22) will vanish identically if the states $\left|\psi_{1}(x)\right\rangle$ and $\left|\psi_{2}(x)\right\rangle$ have the same parity. Labeling the eigenstates in increasing order of energy by the numbers $n$ starting with the number 1 , and noting that alternate states have opposite parity leads to a selection rule that only transitions between states for which $\Delta n=$ odd number are "allowed." In an actual QW, which is doped and nominally rectangular, different degrees of surface roughness will, in general, exist at the two interfaces. This follows from the well-known fact that the quality of the interface resulting from the growth of semiconductor A on semiconductor B is not, in general, the same as the quality of the interface resulting from the growth of B on A. Yet again, any asymmetry in the doping profile will destroy the inversion symmetry. Hence, in an actual nominally rectangular but doped QW structure, the selection rule will be "relaxed," a feature which is exacerbated if an electric field (whether internal or external) exists across any part of the structure. The purpose of these considerations is to highlight the "vulnerability" of supposed selection rules to differences between actual QW structures and ideal QW structures.

Next let us consider a light beam polarized along the $x$ direction (i.e., normal incidence). For this situation we readily obtain, in place of Eq. (3.23) 


$$
\begin{array}{r}
\left\langle\psi_{1}(x, y, z)\left|\frac{\partial}{\partial z}\right| \psi_{2}(x, y, z)\right\rangle=a_{c}^{*} b_{c}\left[\left\langle\chi_{c}(z) \mid \chi_{c}^{\prime}(z)\right\rangle\left\langle U_{c}\left|\frac{\partial}{\partial z}\right| U_{c}\right\rangle+\right. \\
i k x \chi c z \chi c^{\prime} z U c U c \\
=a_{c}^{*} b_{c}\left[\left\langle\chi_{c}(z) \mid \chi_{c}^{\prime}(z)\right\rangle\left\langle U_{c}\left|\frac{\partial}{\partial z}\right| U_{c}\right\rangle+i k_{x}\right]
\end{array}
$$

Hence, if $\left\langle\chi_{c}(z) \mid \chi_{c}^{\prime}(z)\right\rangle=0$ (as is usually assumed in the literature), the matrix element vanishes identically. This is the basis of the polarization selection rule so widely quoted in the literature. For future reference we note that if $\left\langle\chi_{c}(z) \mid \chi_{c}^{\prime}(z)\right\rangle \neq 0$ there are two further terms that could influence the magnitude of the matrix element. The first involves the matrix element of $\partial / \partial z$ between the Bloch function states. As mentioned earlier, although this vanishes if $U_{c}$ is, in fact, the same Bloch state corresponding to $k=0$, we will show later that this matrix element does not vanish if the $U_{c}$ refers to different Bloch states. [This means that, although this term may vanish for transitions between low-lying states for which the effective mass approximation

holds, it will not necessarily vanish in general for a transition between either two highlying states (for which nonparabolicity effects are important) or for a transition between a low-lying state (well described by the effective mass approximation) and a high-lying state (for which

nonparabolicity effects are important).] The second term of relevance involves the inplane wave vector $k_{x}$. Clearly, at higher doping levels the values of $k_{x}$ will increase, and in consequence so too, in general, will the magnitude of the transition dipole matrix element. 
In view of these considerations it is clear that, within the approximations that lead to Eq. (3.22), a key issue is whether or not the matrix element $\left\langle\chi_{c}(z) \mid \chi_{c}^{\prime}(z)\right\rangle$ vanishes. It is this issue we will turn to in the next section.

$$
\begin{aligned}
& \left(E_{1}-E_{2}\right)\left\langle\chi_{2} \mid \chi_{1}\right\rangle=\left\langle\chi_{2}\left|\frac{\hbar^{2}}{2}\left(\frac{\partial^{2}}{\partial z^{2}} \frac{1}{m^{*}(z)}\right)+\hbar^{2}\left(\frac{\partial}{\partial z} \frac{1}{m^{*}(z)}\right) \frac{\partial}{\partial z}\right| \chi_{1}\right\rangle \\
& \left(E_{2}-E_{1}\right)\left\langle\chi_{1} \mid \chi_{2}\right\rangle \\
& =\left\langle\chi_{1}\left|\frac{\hbar^{2}}{2}\left(\frac{\partial^{2}}{\partial z^{2}} \frac{1}{m^{*}(z)}\right)+\hbar^{2}\left(\frac{\partial}{\partial z} \frac{1}{m^{*}(z)}\right) \frac{\partial}{\partial z}\right| \chi_{2}\right\rangle
\end{aligned}
$$

Subtracting these last two equations and noting that $\left|\chi_{1}(z)\right\rangle$ and $\left|\chi_{2}(z)\right\rangle$ are both real functions shows that

$$
\left(E_{1}-E_{2}\right)\left\langle\chi_{2} \mid \chi_{1}\right\rangle=\frac{\hbar^{2}}{2}\left[\left\langle\chi_{2}\left|\left(\frac{\partial}{\partial z} \frac{1}{m^{*}(z)}\right)+\frac{\partial}{\partial z}\right| \chi_{1}\right\rangle-\left\langle\chi_{1}\left|\left(\frac{\partial}{\partial z} \frac{1}{m^{*}(z)}\right)+\frac{\partial}{\partial z}\right| \chi_{2}\right\rangle\right]
$$

It follows from Eq. (3.26) that the overlap integral $\left\langle\chi_{2} \mid \chi_{1}\right\rangle$ will not, in general, vanish. Consider first the hypothetical situation of a perfect, rectangular QW. There is a step function change of magnitude $\Delta$ say, in the value of the inverse effective mass $\left(1 / m^{*}\right)$ at the well-barrier interface region. Consequently, we will have a $\delta$ function there coming from the derivative of the step function. As we move from left to right across the QW, if $\Delta$ is positive at the first interface it will be negative at the second one. Thus,

$$
\left(\frac{\partial}{\partial z} \frac{1}{m^{*}(z)}\right)=\Delta \delta(x+a)-\Delta \delta(x-a)
$$

where we have assumed that the origin of coordinates is at the center of the QW which extends from $z=-a$ to $z=+a$. Given that the states $\left|\chi_{1}(z)\right\rangle$ and $\left|\chi_{2}(z)\right\rangle$ for a perfect, rectangular QW have a definite parity, it is clear that the matrix element 


$$
\left\langle\chi_{2}\left|\frac{\partial}{\partial z} \frac{1}{m^{*}(z)} \frac{\partial}{\partial z}\right| \chi_{1}\right\rangle
$$

will vanish identically if $\left|\chi_{1}(z)\right\rangle$ and $\left|\chi_{2}(z)\right\rangle$ are of opposite parity. The reason for this is simple. If $\left|\chi_{1}(z)\right\rangle$ is of opposite parity to $\left|\chi_{2}(z)\right\rangle$ then $(\partial / \partial z)\left|\chi_{1}(z)\right\rangle$ has identical parity with $\left|\chi_{2}(z)\right\rangle$. Hence, the product term $\chi_{2} \partial \chi_{1} / \partial z$ is of even parity, which means its value at $z=-a$ will be the same as its value at $z=+a$, thus making the matrix element identically zero. Hence, in terms of the present approach, in a perfect rectangular QW the overlap integral $\left\langle\chi_{2} \mid \chi_{1}\right\rangle$ vanishes identically for states of opposite parity and we can expect negligible normal incidence absorption between adjacent levels in the QW, since such levels are of opposite parity. However, the overlap integral will not vanish for states having the same parity, although its value may well be small.

For a conduction-band electron the effective mass, in general, varies only weakly (and usually linearly) with the material composition. As a result, by making a binomial expansion, it is not unreasonable to expect that, in general, in the graded-gap region of the well

$$
\frac{1}{m^{*}(z)}=\mathrm{a}+\mathrm{bz}
$$

where the $z$ dependence comes from the linear dependence of the physical composition of the well material on the position $z$ in the QW. For such a situation we readily obtain

$$
\frac{\partial}{\partial \mathrm{z}}\left(\frac{1}{\mathrm{~m}^{*}(\mathrm{z})}\right)=\Delta \delta(\mathrm{x}+\mathrm{a})-\Delta_{1} \delta(\mathrm{x}-\mathrm{a})+\mathrm{b}
$$


where the two delta functions arise from the discontinuous change in the effective mass at the interfaces of the well boundary region. Apart from the two delta-function contributions, we see that the term involving $b$ on the rhs of Eq. (3.30) gives a contribution to Eq. (3.29) of the form

$$
\left(E_{1}-E_{2}\right)\left\langle\chi_{2} \mid \chi_{1}\right\rangle=\hbar^{2} b\left\langle\chi_{2}\left|\frac{\partial}{\partial z}\right| \chi_{1}\right\rangle,
$$

where we have utilized the fact that for two real functions integration by parts shows that

$$
\left\langle\chi_{2}\left|\frac{\partial}{\partial z}\right| \chi_{1}\right\rangle=-\left\langle\chi_{1}\left|\frac{\partial}{\partial z}\right| \chi_{2}\right\rangle \text {. }
$$

The interesting feature of Eq. (3.31) is that the matrix element on the rhs is exactly that which occurs for light propagating along the $z$ direction as discussed earlier. One final point to note from Eq. 3.31 is that, other things being equal, the overlap integral will increase for closer energy spacing of the two states concerned, i.e., in general, normal incidence absorption would be expected to occur more readily for far infrared radiation than for near-infrared radiation.

Direct bandgap III-V compound semiconductor material systems, such as AlGaAs/InGaAs and AlGaAs/nAlAs, are popular choices for n-type quantum well infrared photodetectors (QWIPs) [1, 2]. As has been mentioned earlier, electrons in such material systems occupy states around the $\Gamma$ valley, and since the effective mass of the electrons in the $\Gamma$ valley is small, the high mobility of the electrons can provide a large photoresponsivity in the QWIP. One major drawback of these material systems is that the $\Gamma_{6}$ symmetry of the lowest antibonding conduction band basically forbids intersubband transitions induced by transverse electric (TE) polarized infrared radiation. However, the 
parity selection rule governing the transitions does not hold strictly because of the effect of band mixing as a result of the truncation of the periodic bulk structure, the spatial variation of the material parameters as well as the formation of subbands in multiple quantum wells (MQWs). Nevertheless, the response of the symmetrical quantum wells (QWs) to TE excitation is generally very small compared to that due to transverse magnetic (TM) excitation.

The parity selection rule can be further broken by modifying the structure of the QW. Instead of a symmetrical square QW, an asymmetrical QW could be adopted instead. In such a structure, the envelope wavefunctions do not have a definite odd or even parity, and therefore could, in principle, allow for a larger TE response. This would be very useful as it would facilitate the fabrication of focal plane arrays for normal incident absorption without the need for additional coupling schemes such as grating structures. In this thesis, the factors that affect TM and TE absorptions, such as the probability density distributions of the wavefunctions, and their coupling strengths, are investigated in terms of the material and structural parameters of the step QW, namely, the depth of the well and the width and height of the step. Through a better understanding of the mechanisms governing the intersubband transitions in such devices, it may then be possible to design an optimum structure to enhance normal incident absorption.

\subsection{NEGF and Quantum Wave Impedance}

Non-equilibrium Green's function (NEGF) and concept of quantum mechanical wave impedance are used to perform the quantum mechanical analysis of asymmetric quantum well structures and to deduce their optical absorption properties. This section 
outlines the theoretical framework for calculating eigen states and their associated wavefunctions, and density of states (DOS). The starting point of this scheme is the Schrodinger equation modified by the presence of the optical (local) potential:

$$
\left(E_{x}-\mathrm{H}_{0}(x)+\frac{i \hbar}{2 \tau_{\phi}\left(x, E_{x}\right)}\right) G^{R}\left(x, x_{1}, E_{x}\right)=\delta\left(x-x_{1}\right)
$$

The Hamiltonian $\mathrm{H}_{0}(x)$ contains the potential-energy term that is determined by the band discontinuity and the self-consistent Hartree potential, and $\tau_{p}\left(x, E_{x}\right)$ and $\tau_{\phi}\left(x, E_{x}\right)$ are the electron in-scattering time and the phase-breaking time, respectively. The magnetic vector potential is ignored in this work. The function $G^{R}\left(x, x_{1}, E_{x}\right)$ therefore contains all the interference effects due to elastic scattering. Conventionally, the Green's function is expressed in terms of a complete set of eigenfunctions. We will, however, solve for the Green's function $G^{R}\left(x, x_{1}, E_{x}\right)$ using a different approach. Let us define a function $Z\left(x, x_{1}, E_{x}\right)$ as

$$
Z\left(x, x_{1}, E_{x}\right)=\left(\frac{2 \hbar}{i m^{*}} \frac{\partial G^{R}\left(x, x_{1}, E_{x}\right)}{\frac{\partial x}{G^{R}\left(x, x_{1}, E_{x}\right)}}\right)
$$

It can be shown from Eq. (3.34) that

$$
\frac{\hbar}{m^{*}} \nabla \theta\left(x, x_{1}, E_{x}\right)=\frac{\operatorname{Re}\left[Z\left(x, x_{1}, E_{x}\right)\right]}{2}
$$

which has the units of velocity. In order to calculate $Z\left(x, x_{1}, E_{x}\right)$ we have to use two different boundary conditions because $Z\left(x, x_{1}, E_{x}\right)$ has a discontinuity at $x=x_{1}$. The boundary conditions are, respectively, as follows: 
$Z\left(x=\infty, x_{1}, E_{x}\right)=Z_{0}(\infty)$ for $x>x_{1}$ and $Z\left(x=-\infty, x_{1}, E_{x}\right)=-Z_{0}(-\infty)$ for $x<x_{1}$. Here $Z_{0}(x)=\left(2 \hbar / m^{*}\right) \gamma(x)$ and This is so because as $x \rightarrow \infty, G^{R}\left(x, x_{1}, E_{x}\right) \approx c_{1} e^{\gamma_{1} x}$ where $\gamma_{1}=\gamma(\infty)$ is a constant. Similarly for $x \rightarrow-\infty$ we have $G^{R}\left(x, x_{1}, E_{x}\right) \approx c_{2} e^{-\gamma_{2} x}$ where $\gamma_{2}=\gamma(-\infty)$. Here $\gamma_{1}$ and $\gamma_{2}$ are calculated inside the left and right reservoirs, respectively, where the potential energy is constant. The method for the calculation of a quantity similar to $Z\left(x, x_{1}, E_{x}\right)$ has been discussed elsewhere. The use of two boundary conditions reveals an interesting property of the function $Z\left(x, x_{1}, E_{x}\right)$. Since $Z\left(x, x^{\prime}, E\right)$ has a discontinuity at $x=x^{\prime}$, one needs two boundary conditions. These are: $Z\left(x=\infty, x^{\prime}, E\right)=Z_{0}(\infty)$ for $x>x^{\prime}$ and $Z\left(x=-\infty, x^{\prime}, E\right)=-Z_{0}(-\infty)$ for $x<x^{\prime}$. Here

$$
\begin{aligned}
& Z_{0}(x)=\frac{2 \hbar}{i m^{*}} \gamma(x), \text { and } \\
& \gamma(x)=i\left(\frac{2 m^{*}}{\hbar^{2}}[E-V(x)+i \in]\right)^{1 / 2}
\end{aligned}
$$

where $\epsilon$ is an infinitesimally small positive energy. When the two boundary conditions are used, it can be shown that, for all $x^{\prime}<x$ :

$$
Z\left(x, x^{\prime}, E\right)=Z^{+}(x, E),
$$

and for all $x^{\prime}>x$ :

$$
Z\left(x, x^{\prime}, E\right)=Z^{-}(x, E) \text {. }
$$

It is worth noting that $Z^{+}(Z)$ does not depend on $x^{\prime}$ as long as $x>x^{\prime}\left(x<x^{\prime}\right) . Z^{ \pm}$are calculated using a method analogous to the impedance transformation technique of transmission lines along with the two boundary conditions discussed above. 


\subsubsection{Calculation of quantum wave impedance}

The well-developed theory of transmission lines can be effectively used to calculate the quantum mechanical transmission probability, This can be achieved by introducing an impedance analog for the quantum mechanical wave. In order to introduce and define the concept of wave impedance we use a simple potential barrier (Figure: 3.3). Arbitrary potential barrier structures will be used later to show the capability of this method. Let us assume that an electron with an energy $E$ is incident on the potential barrier, as shown in the figure. The wave function $\psi(x)$, in general, can be written as

$\psi(x)=A^{+}\left(e^{2 x}-\rho e^{-\gamma x}\right)$

where

$\gamma=\alpha+j \beta=j \sqrt{\left(2 m^{*} / \hbar^{2}\right)(E-V)}$

is the propagation constant, $m *$ is the effective mass, $j=\sqrt{ }-1, \hbar$ is the modified Plank's constant, $V$ is the potential, and $\rho$ is the wave amplitude reflection coefficient. Note that in Eq. (3.38) the time variation is implicity assumed as $\exp (-j E t / \hbar)$. Now, for the two regions shown in Figure: 3.3, Eq. (3.38) may be written as

$$
\begin{array}{ll}
\psi_{1}(x)=A_{1}^{+}\left(e^{\gamma_{1} x}-\rho e^{-\gamma_{1} x}\right), & x<0 \\
\psi_{2}(x)=A_{2}^{+} e^{\gamma_{2} x}, & x>0, \\
\text { where } \gamma_{i}=j \sqrt{\left(2 m_{i}^{*} \hbar^{2}\right)\left(E-V_{i}\right)}=\alpha_{i}+j \beta_{i}, m_{i}^{*}
\end{array}
$$




\section{incident}

electron

transmitted

uns

reflected

electron

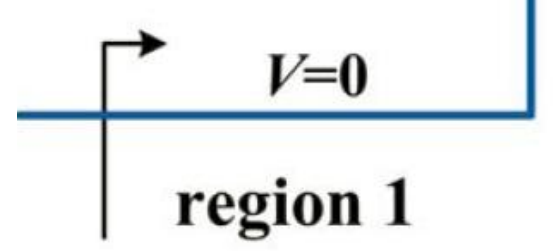

region 2

$Z_{i}$

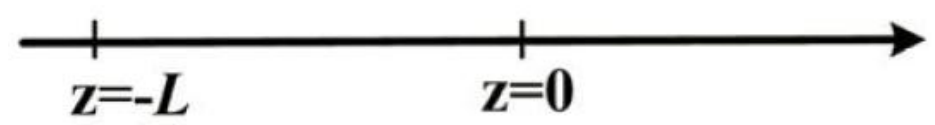

Figure 3.3: A step potential barrier illustrating the concept of quantum wave impedance.

and $V i$ and $m_{i}^{*}(i=1,2)$, are the potential and effective mass respectively, for the $i$-th region. It is implied that there is no reflection of $x>0$ because the region is of infinite extent. Now applying the boundary conditions that $\psi_{1}^{\prime}(0) / m^{*}=\psi_{2}^{\prime}(0) / m^{*}$ and $\psi_{1}(0)=$ $\psi_{2}(0)$, we get an expression:

$\rho=\left[\left(\gamma_{2} / m_{2}^{*}\right)-\left(\gamma_{1} / m_{1}^{*}\right)\right] /\left[\left(\gamma_{2} / m_{2}^{*}\right)+\left(\gamma_{1} / m_{1}^{*}\right)\right]$

This equation is analogous to the wave amplitude reflection coefficient defined in transmission line equations. In order to show this analogy with transmission lines we differentiate Eq. (3.38) with respect to $x$ and multiply both sides by a factor $\hbar / i m{ }^{*}$ to obtain 
$\phi(x)=\frac{\hbar}{j m^{*}} \frac{d \psi}{d x}=A^{+} Z_{0}\left(e^{\gamma x}+\rho e^{-\gamma x}\right)$,

where $Z_{0}=\hbar \hbar / j m^{*}$.

At any plane $x$, we define the quantum mechanical wave impedance as $Z(x)=\phi(x) / \psi(x)$. Thus, the input value of impedance, $\mathrm{Zi}=\mathrm{Z}(-I)$, may be expressed in terms of the load impedance $\mathrm{Z}_{\mathrm{l}}=\mathrm{Z}(0)$

$Z_{i}=Z_{0} \frac{Z_{l} \cosh (\gamma l)-Z_{0} \sinh (\gamma)}{Z_{0} \cosh (\gamma l)-Z_{l} \sinh (\gamma)}$

The load impedance seen by the first region of Figure:3.3 at $x=0$ is the input impedance $\mathrm{Zi}$ of region 2 at $x=\mathrm{O}$.

\subsubsection{Eigen functions and DOS}

The eigenenergies of an arbitrary quantum well can easily be determined from

$Z^{ \pm}$as we know that at any eigenenergy $E n$

$$
Z^{+}\left(x, E_{n}\right)=Z^{-}\left(x, E_{n}\right)
$$

for all values of $x$ inside the quantum well. An attractive part of this method is that, once the eigenenergies have been found, the normalized wave functions can be obtained without much effort. To show this, we use the well known expansion of $G^{R}$ in terms of the complete set of eigenfunctions:

$$
G^{R}\left(x, x^{\prime} ; E\right)=\sum_{m} \frac{\psi_{m}(x) \psi_{m}^{*}\left(x^{\prime}\right)}{E-E_{m}+i \in} .
$$

Here $\psi_{m}(x)$ is the normalized wave function corresponding to the $m$ th eigenenergy $E_{m}$. If $E_{m+1}-E_{m} \gg \uparrow$ for all values of $m$, only one term in the series dominates when $E \rightarrow E_{n}$, 
since the discrete eigenenergies in one-dimension are nondegenerate. For the diagonal elements of $G^{R}$, we obtain

$$
G^{R}\left(x, x ; E \rightarrow E_{n}\right) \cong \frac{\mid \psi_{n}\left(\left.x\right|^{2}\right.}{E-E_{n}-i \in} .
$$

Taking imaginary parts of both sides of Eq. (3.49), and substituting $E=E_{n}$,

$$
\left|\psi_{n}(x)\right|^{2}=-\in \operatorname{T} m\left[G^{R}\left(x, x ; E_{n}\right)\right]
$$

It can also been shown that

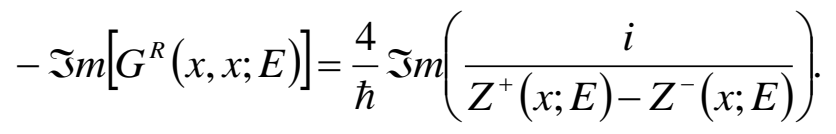

Substitution of Eq. (3.49) in Eq. (3.51) gives us

$$
\left|\psi_{n}(x)\right|^{2}=\frac{4 \in}{\hbar} \Im m\left(\frac{i}{Z^{+}\left(x ; E_{n}\right)-Z^{-}\left(x ; E_{n}\right)}\right) .
$$

It should be pointed out that since the values of $Z^{ \pm}$are needed to be calculated for all $x$ for estimating the eigenenergies $E_{n}$ using Eq. (3.47), Eq. (3.52) then allows us to calculate the normalized wave functions $\left|\psi_{n}(x)\right|^{2}$ as a byproduct. While it may seem that $\left|\psi_{n}(x)\right|^{2}[\mathrm{Eq} .(3.52)$ depends on the choice of $\epsilon$, the result is actually independent of $\epsilon$. This is because, for sufficiently small $\left.\epsilon, \mathrm{T}\left\{\frac{i}{\left[Z^{+}\left(x ; E_{n}\right)-Z^{-}\left(x ; E_{n}\right)\right.}\right]\right\}$ itself scales linearly with $1 / \epsilon$. The 1D density of states (DOS), $N_{\text {Dos }}$, is related to the diagonal part of $G^{R}$ and can be expressed in terms of logarithmic derivatives $Z^{ \pm}$

$$
\begin{gathered}
N_{1 D}(z ; E)=-\frac{1}{\pi} \Im m\left[G^{R}(z, z ; E)\right] \\
=\frac{4}{\pi \hbar} \Im m\left[\frac{i}{Z^{+}(z ; E)-Z^{-}(z ; E)}\right] .
\end{gathered}
$$




\section{$N_{\text {DOS }}(E)$}

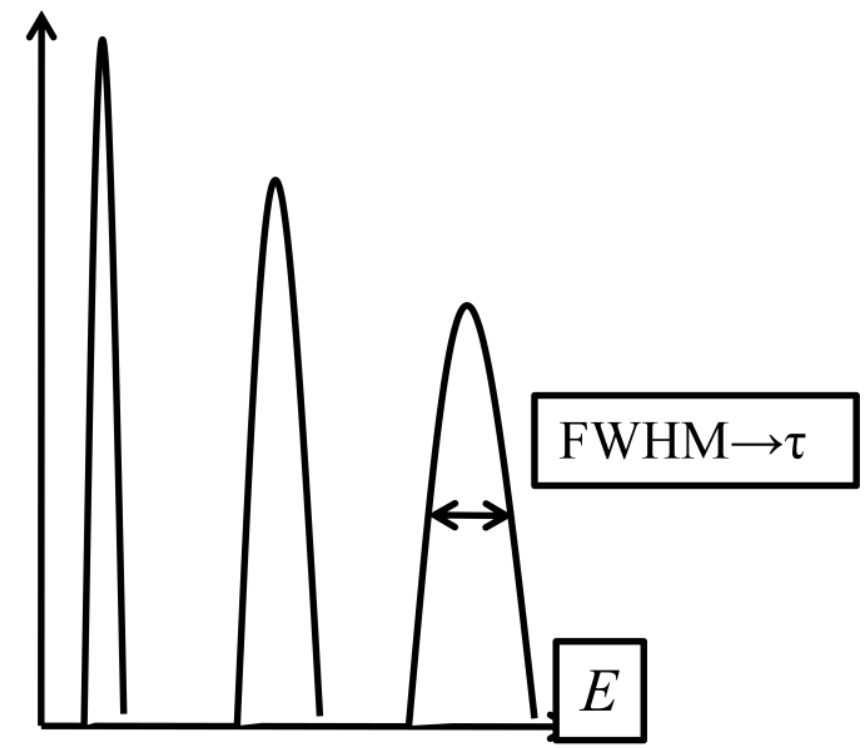

Figure:3.4. Representation of DOS as a function of energy that reveals the broadening of energy states and FWHM, along with carrier lifetime on a given energy state.

When carriers (electrons) tunnel out of any energy state, the carrier lifetimes become finite and induces broadening of the DOS around the respective eigenenergy, as shown in Figure: 3.4. Tunneling results in complex values for eigenenergies. The real part of the complex eigenenergy gives the energy of the quasibound state and the imaginary part is related to the lifetime of the carriers. To avoid numerical determination of complex eigenvalues of a non-Hermitian Hamiltonian, we apply a different, yet simple approach. The energies of the quasibound states, $E n$, are calculated by locating the peaks of $N_{1 \mathrm{D}}$ as a function of energy, evaluated using Eq (3.53). The lifetimes, of the inversion layer carriers are obtained from the calculated full width at half maximum (FWHM), $\Gamma$, of the 
energy broadened DOS around the $n$th eigenenergy using the relationship $\tau=\hbar / 2 \Gamma$. The FWHM can be estimated anywhere inside the potential well as it has been shown that the energy broadening of the DOS is the same at all positions.

\subsection{Conclusion}

This chapter presents and details the key concepts used in this dissertation to quantify the absorption of infrared radiation in asymmetric quantum well structure. The mathematical models described here have been used to analyze III-V material based quantum well structures to achieve optimized photodetection efficiency that are described in the next chapter. One of the main reasons behind choosing III-V material system such as $\mathrm{AlGaAs} / \mathrm{GaAs} / \mathrm{InGaAs}$ systems is the option of devising $n$-type device with higher mobility that potentially leads to improved responsively. Unfortunately electrons in these materials occupy $\Gamma$ valley of conduction band and this results in certain symmetries in wavefunctions. The wavefunction symmetry or parity prohibits transition among certain eigen states. This selection rule is mostly dominant in square quantum wells. This is one of the key reasons that the response of a square QW to normal incident radiation is very weak. Therefore, to break the symmetry of a simple square QW, an asymmetric step QW design is a good candidate for improving the absorption. A detail theoretical description of selection rule has been presented. It has been shown mathematically how asymmetric quantum wells can break the symmetry of wavefunctions and hence facilitate the absorption of normal incidence or transverse electric (TE) wave.

Starting from defining a scattering potential that is felt by an electron in presence of photon, it has been shown how absorption rate is calculated using Fermi Golden rule 
using the concept of broadening of density of states. The effect of strain that modifies the bandgap of the constituent materials and therefore the overall quantum well structure has been described. The calculation of eigen energies and associated wavefunctions have been calculated using NEGF and the concept of quantum wave impedance, which is based on well developed microwave transmission line theory. 


\section{CHAPTER 4}

\section{Analysis of Asymmetric Quantum Wells for Infrared Photodetection}

In this chapter we present detailed description of the investigation on asymmetric quantum well structures having potential applications in infrared photodetection that is based on the physical model described in previous chapters. As has been already detailed, the quantifying scheme developed in this work is based on non-equilibrium Green's function and quantum mechanical wave impedance concept. The main reason that makes the presented methodology very suitable for gaining physical insight regarding the efficacy of a given quantum well structure is it's simplicity. This allows the scheme to be easily programmable without sacrificing the accuracy. Moreover the presented method allows direct calculation of certain parameters like optical broadening, which have been mostly assumed in many previously reported works, a point which has been stressed in chapter 3 and is also discussed in brief in this chapter.

In the following sections of this chapter, the outline of the model is briefly described, which is then followed by some key results obtained for asymmetric quantum well structure based on III-V semiconductors that have been already reported as been investigated. This allows comparing the results obtained here with published works and thus establishes the validity of the developed scheme. The structure that has been extensively investigated here is based on $\mathrm{AlGaAs} / \mathrm{GaAs} / \mathrm{InGaAs}$ that forms a stepped 
quantum well with dimensions tunable with the variation of concentrations of indium (In) and aluminum (Al). The step height and width determines the "asymmetry" of the well that distorts the wavefunction and hence destroys any "parity" condition that the wavefunction may possess due to it's position at $\Gamma$ valley, as has been detailed in chapter 3. The calculated results for optical and tunneling broadening of density of states have been presented to explain the impact of asymmetry of the well. This chapter also presents the impact of electric field and strain on the overall absorption and detection wavelength. The structures capable of detecting multiple wavelengths or "color" using applied electric fields are also analyzed.

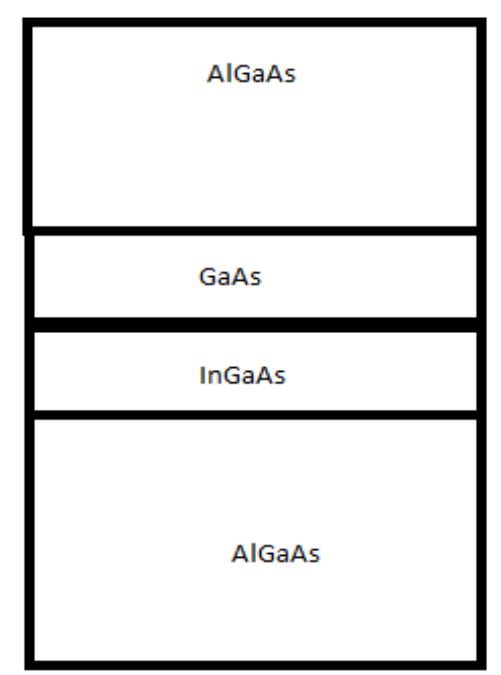

Figure 4.1: Schematic representation of a single period of $A I G a A s / G a A s / l n G a A s$ quantum well structure.

\subsection{Analysis of AIGaAs/GaAs/nGaAs n-type Step Quantum Wells}

To demonstrate the validity of the developed mathematical model and the concept of improving overall infrared absorption using asymmetric wells, we consider the 
conduction band energy profile of an $\mathrm{Al}_{x} \mathrm{Ga}_{1-x} \mathrm{As} / \mathrm{GaAs} / \mathrm{In}_{y} \mathrm{Ga}_{1-y} \mathrm{As}$ material system as shown in Figure 4.1. As previously mentioned, the AlGaAs/GaAs quantum well structures, because of it's lattice matched structure have been preferred by the investigators and hence have matured compared to other QWIPs. To enhance the speed of the detector, the material with high mobility ( $\mathrm{InGaAs}$ ) is used as the well material. However, using indium in the quantum well material means that strain is incorporated in the structure as GaAs and InGaAs are not lattice-matched. While InGaAs is grown epitaxially on GaAs or AlGaAs, first few atomic layers will remain strained to match the previous layer. Once a certain thickness is exceeded, defects will be introduced in the structure. This will undermine the carrier mobility and therefore the layer thickness should be carefully

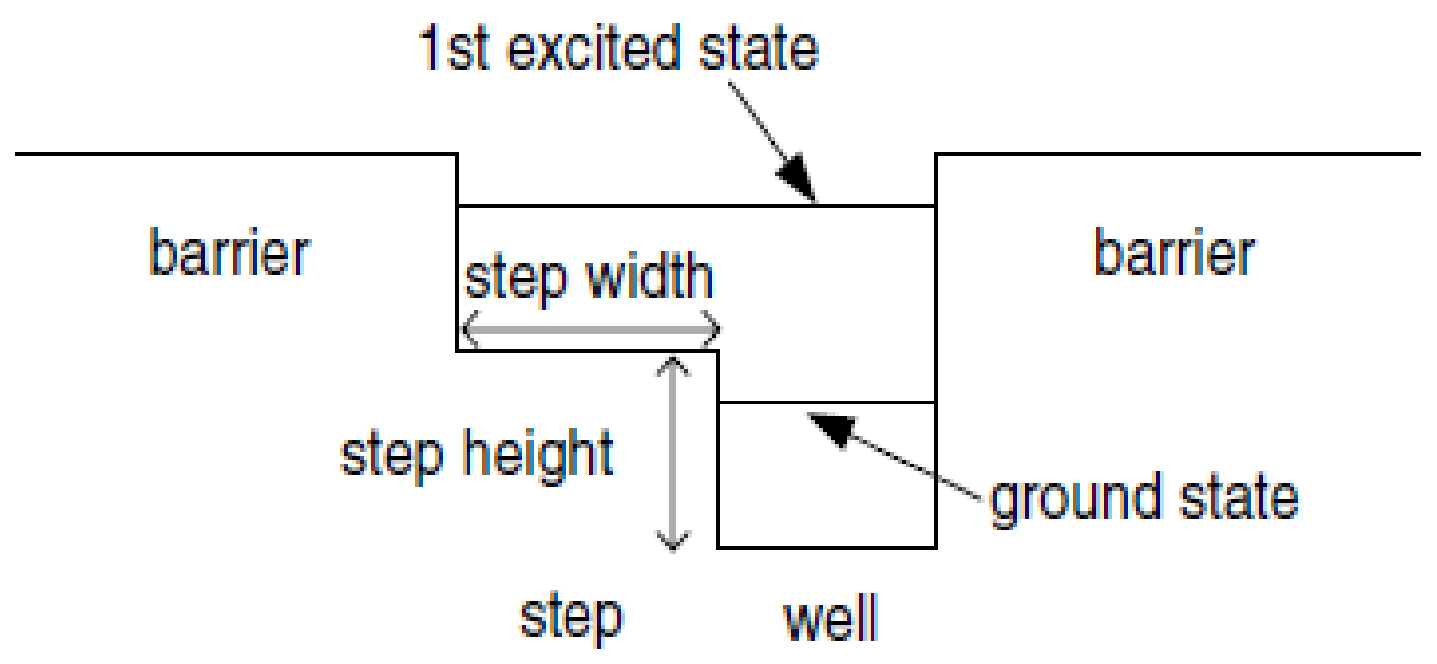

Figure 4.2: Schematic representation of asymmetric/stepped quantum well showing step width and height. 
optimized. The procedure to calculate this optimum thickness is described in later section. GaAs/InGaAs forms the stepped well region to achieve required "asymmetry", whereas the AlGaAs forms the barrier layers. Since inter-subband transition resulting from infrared absorption occurs entirely within conduction band, electron-hole pairs are not created and hence QWs must be doped (in this context $n$-type) to produce detectable photo-current. For this reason, the devices investigated here are generally assumed to have doping concentrations of $10^{18} \mathrm{~cm}^{-3}$ in the well region, along with undoped step and barrier regions.

The schematic diagram of one period of the structure of the step QW is shown in Figure 4.2. In the calculations presented in this section for model validation, the device is assumed to be $n$-type, uniformly doped with a concentration of $2 \times 10^{18} \mathrm{~cm}^{-3}$ in the well region and undoped in the step and barrier regions. The well width, step width and step height are varied to study the effects of these parameters of the step MQW on the behavior of the envelope wavefunctions and the intersubband transitions, in order to elicit those factors that enhance TE absorption. Figure 4.3 shows the calculated conduction band profile and wavefunction for an $\mathrm{Al}_{x} \mathrm{Ga}_{1-x} \mathrm{As} / \mathrm{In}_{y} \mathrm{Ga}_{1-y} \mathrm{As} / \mathrm{GaAs}$ with $x=0.15$ and $y=0.15$.

As can be seen from the spatial distribution of the wavefunction (it is actually wavefunction squared to be exact and therefore can be interpreted as probability density distribution), the ground state is localized mostly in the well region, whereas that of first excited state has it's dominant lobe in the step region. Figure 4.4 also show calculated DOS and absorption coefficient for the structure under consideration. The eigen states are found to be at $\sim 0.068 \mathrm{eV}$ and $\sim 0.1734 \mathrm{eV}$. The corresponding peak of absorption is 
therefore found to be around $0.10575 \mathrm{eV}$. It is worth mentioning that that the effective mass variation of the structure has been taken into consideration. The effective mass for the regions barrier, well and step, were found to be $0.79,0.0659$ and 0.0665 respectively. The broadening due to tunneling is found to be $2 \times 10^{-6} \mathrm{eV}$ where as that for optical absorption is $1 \times 10^{-11} \mathrm{eV}$.
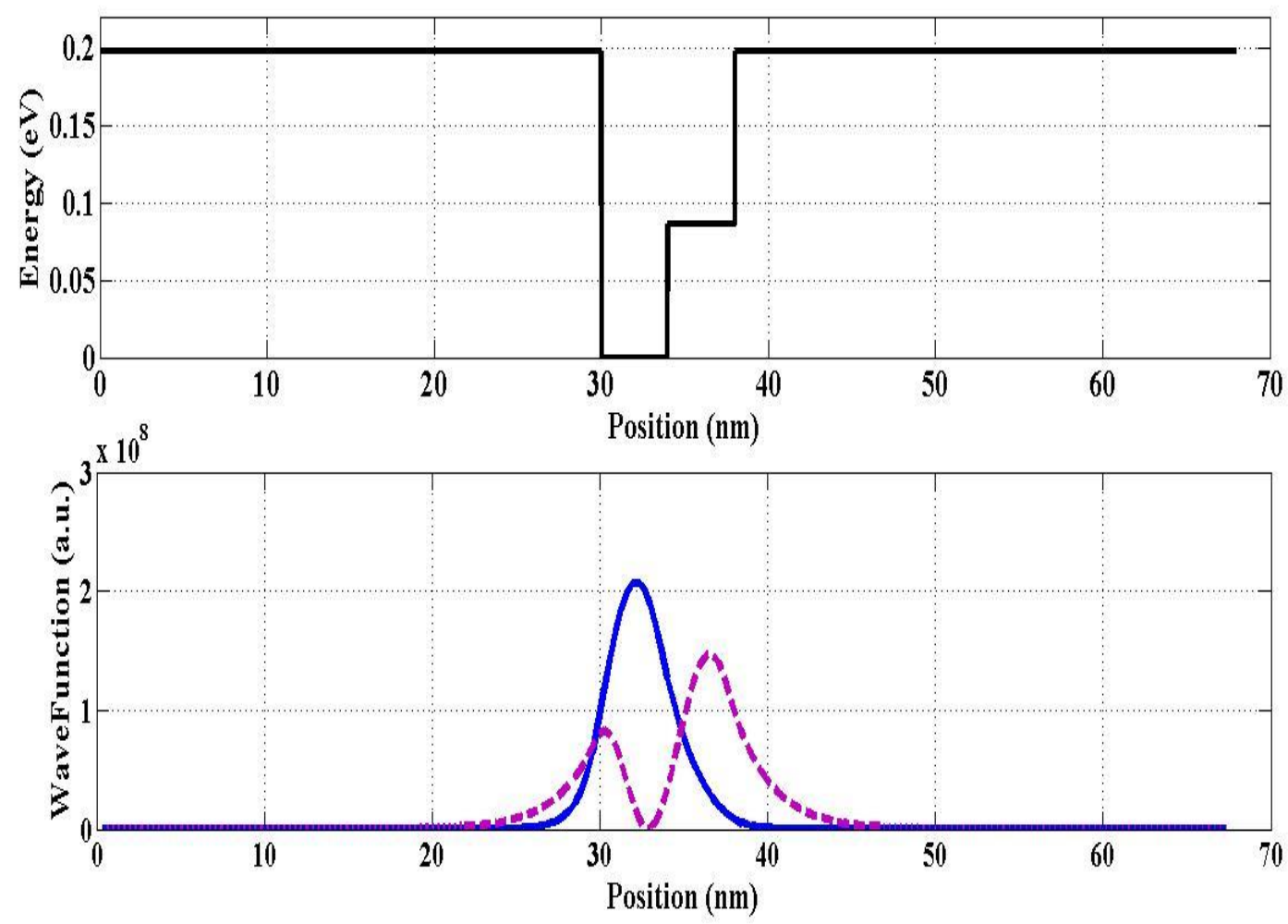

Figure: 4.3 Conduction band profile and wavefunctions for a $\mathrm{Al}_{x} \mathrm{Ga}_{1-x} \mathrm{As} / \ln _{y} \mathrm{Ga}_{1-y} A s$ /GaAs with $15 \% \mathrm{Al}$ and $15 \%$ In concentration. The wavefunctions associated with the ground state and first excited states are shown in blue and purple dotted lines. 

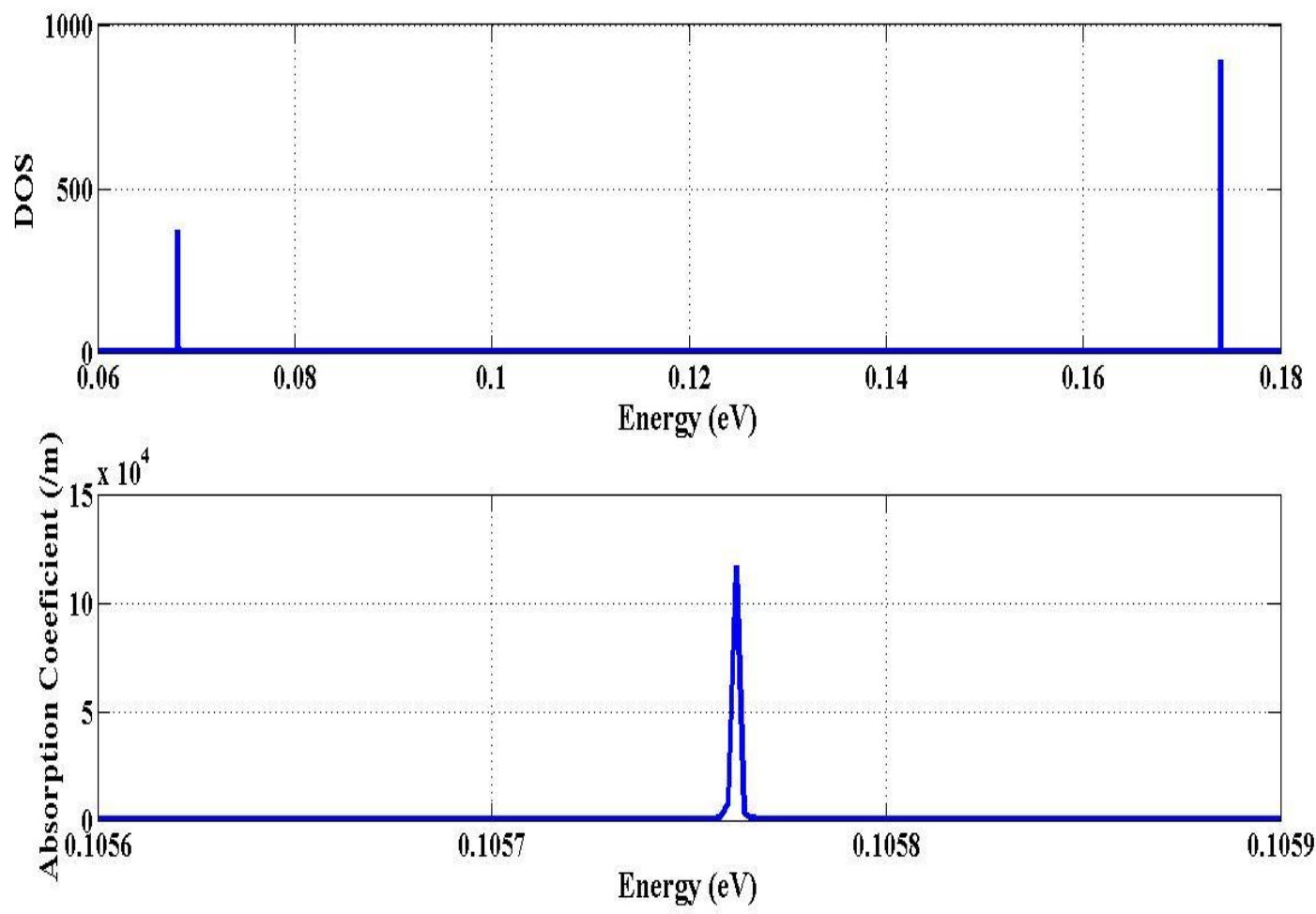

Figure 4.4 Calculated Density of states (DOS) and absorption coefficient for the structure shown in Figure: 4.3.

\subsection{Effect of Asymmetry on Overall Absorption}

It has been already outlined that, the mesoscopic representation of the quantum processes that govern both vertical (absorption) and lateral transport (tunneling) of electrons in an asymmetric quantum well structure, has been utilized to quantify QWIP performance parameters in this work. Also it has been detailed in chapter 3 that, due to the scattering potential felt by electrons in presence of photons electric field, calculation of wavefunctions only at eigen energies becomes inadequate in such cases. The work reported here, therefore, determines the density of states (DOS) as a function of energy 
using non-equilibrium Green's function(NEGF) formalism and quantum mechanical wave impedance concept [63]. It will be evident that the approach has two distinct advantages. First, it is accurate, easily programmable and yet computationally efficient. Second, it facilitates quantifying the broadening of states resulting from both photon absorption and tunneling, which provides important insight for improving detection efficiency. Instead of being presented through calculations, such broadening has been simply assumed in previously reported works.

The results presented in this section reveals how the asymmetry of a quantum well, in this case achieved through steps, destroys the parity or symmetry of the wavefunctions and hence impact the overall absorption performance of the structure. As detailed in Ref [31], imaginary part of $G^{R}$ can be expressed as

$$
\mathfrak{S} m\left[G^{R}\left(x, x_{n}, E_{m}\right)\right]=\frac{4}{\hbar} \mathfrak{I} m\left[\frac{1}{Z_{i R}\left(x, E_{m}\right)-Z_{i L}\left(x, E_{m}\right)}\right]
$$

Here $E_{m}$ is an eigen state. The spatial distribution of wave function and the DOS functions are then very easily calculated.

$$
\begin{gathered}
\left|\psi_{m}(x)\right|^{2}=0^{+} \operatorname{Jm}\left[G^{R}\left(x, x_{n}, E_{m}\right)\right] \\
N_{D O S}(x, E)=\frac{4}{\pi \hbar} \operatorname{Im}\left[G^{R}\left(x, x_{n}, E_{m}\right)\right]
\end{gathered}
$$

Note that the DOS at an eigen energy is calculated as a function of energy $(E)$. The peaks of $N_{D O S}(E)$ give the eigen energies while the full width at half maxima of the peaks provide $\Gamma$ of the state due to electrons tunneling out of the state. With $N_{D O S}(E)$ and $\rho_{i f}$ known, the broadening of the given state due to optical absorption is calculated (eqn. 1). 
Therefore, unlike previous works where $\Gamma$ is simply assumed [48],[64], the scheme presented here provides an effective way to calculate $\alpha$ in terms of broadening resulting from photon absorption. This formulation also yields relative broadening of a state due to optical absorption $\left(\Gamma_{o p}\right)$ and tunneling $\left(\Gamma_{\text {tun }}\right) . \Gamma_{o p} / \Gamma_{\text {tun }}$ can be used as a design parameter to increase the absorption, since it represents the probability of an electron's absorbing a photon before escaping the quantum well. Here also we consider the conduction band energy profile of an $\mathrm{Al}_{x} \mathrm{Ga}_{1-x} \mathrm{As} / \mathrm{In}_{y} \mathrm{Ga}_{1-y} \mathrm{As} / \mathrm{GaAs}$ material system as shown in Figure 4.5. For this analysis we have chosen a barrier/well combination of $30 \mathrm{~nm} / 12 \mathrm{~nm}$. $30 \mathrm{~nm}$ barrier and $12 \mathrm{~nm}$ well combination was chosen to ensure that QW structures can have at least 3 bound states, such that the impact of wavefunction symmetry breaking achieved through stepped QW can be revealed. Results are presented to show that with stepped QW, transition from $1^{\text {st }}$ to $3^{\text {rd }}$ excited state actually becomes dominant. Dimensions other than $30 \mathrm{~nm} / 12 \mathrm{~nm}$ will have shifted positions for eigen energies leading to different values of $\rho_{\text {if. }}$ But for any barrier/well combination that are large enough to support minimum three bound states, similar results can be obtained that shows the impact of symmetry breaking on facilitating transitions forbidden by the selection rule.

The probability distribution calculated across the structure for the bound states are shown in Figure: 4.5 for both stepped and square well. The breaking of wavefunctions symmetry in case of stepped or asymmetric well is clearly evident. The wavefunctions symmetry is very important as it leads to certain selection rules for photon absorption. According to eqn. (4.2), $\rho_{i f}$ depends on the overlapping integral of final state $\left(\psi_{f}\right)$ and the gradient of initial state $\left(\psi_{i}\right)$ in the direction of light polarization. This is correct for incident light with TM polarization that has E-field component along the growth 
direction. However for normal incidence or TE polarization, E-field is perpendicular to the growth direction, in which the wavefunctions are confined. For instance, in the case when $\psi_{i, f}$ are functions of $x$ direction, we can assume normally incident light to be

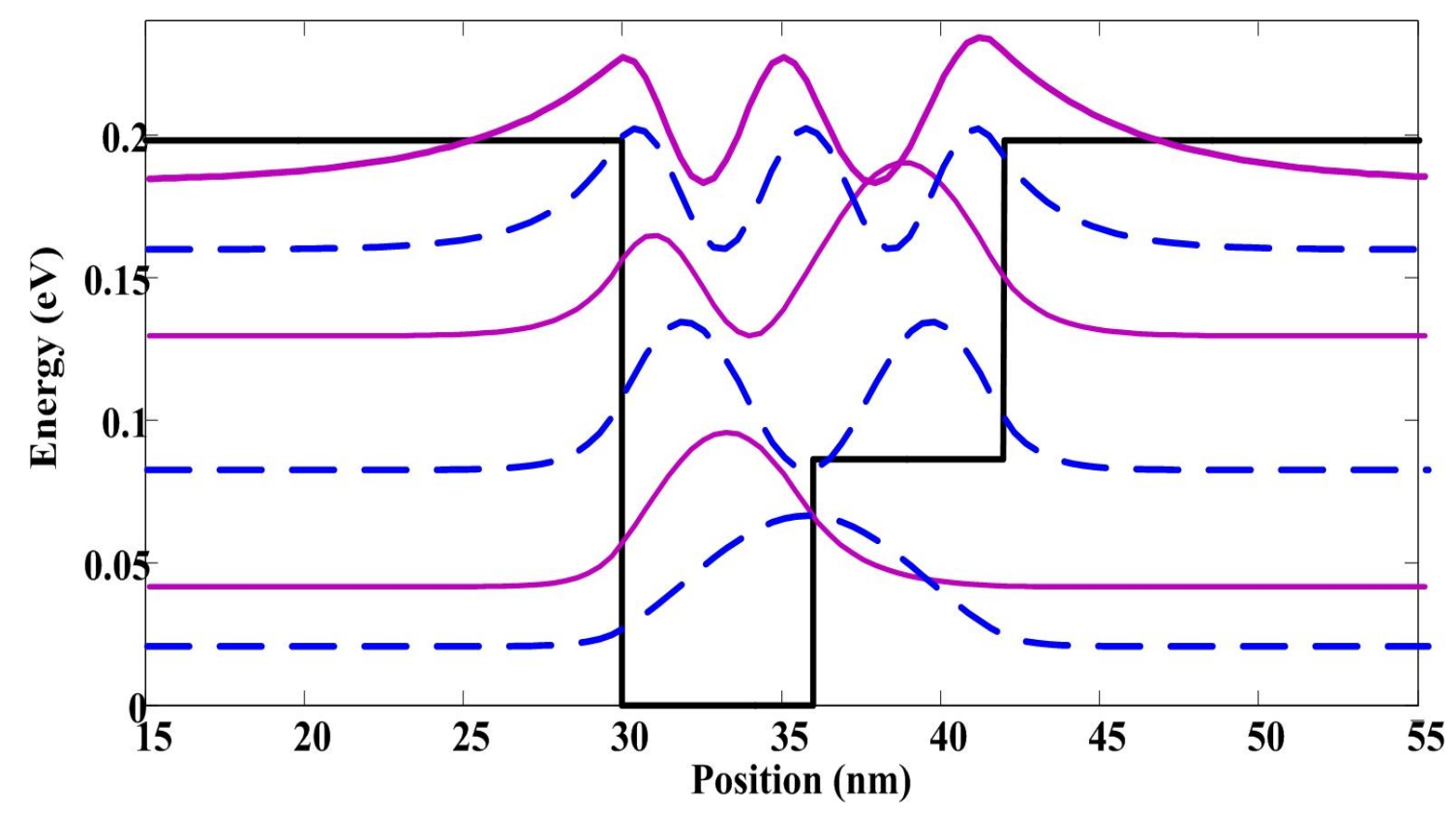

Figure 4.5: Calculated magnitudes of wavefunctions (with their energies shown on the vertical axis) for $\mathrm{Al}_{0.15} \mathrm{Ga}_{0.85} \mathrm{As} / \ln _{0.15} \mathrm{Ga}_{0.85} \mathrm{As} / \mathrm{GaAs}$ structure. The blue dotted lines are for rectangular $\mathrm{QW}\left(\mathrm{Al}_{0.15} \mathrm{Ga}_{0.85} \mathrm{As} / \mathrm{GaAs}\right)$.

polarized along $y$ or $z$ direction. Therefore $\rho_{i f}$, in case of TE polarization depends not only on the gradient of $\psi_{i}$ in the growth direction, but also on the overlapping integral of two wavefunctions [48]. As has been detailed elsewhere [24], this leads to different selection rule for TE and TM polarization: for TM, $\rho_{i f}$ vanishes if $f-i$ is an even number. For TE polarization, however, $\rho_{i f}$ is zero for all cases. We have calculated $\rho_{i f}$ for a rectangular QW similar to the one shown in Figure:4.5 (30 $\mathrm{nm}$ barrier and $12 \mathrm{~nm}$ well) assuming TM 
polarization. For a square $\mathrm{QW}, \rho_{i f}$ among ground state $|1\rangle$ and second excited state $|3\rangle$ is found to be 0.006 , whereas that between $|2\rangle$ and $|3\rangle$ is 0.114 . Whereas for stepped QW (with $6 \mathrm{~nm}$ step width), $\rho_{i f}$ of $|1\rangle \rightarrow|3\rangle$ and $|2\rangle \rightarrow|3\rangle$ are found to be 0.285 and 0.221 respectively. So, for stepped QW, selection rule forbidden $|1\rangle \rightarrow|3\rangle$ transition actually has the largest coupling constant. Clearly, the selection rule that stem from wavefunctions symmetry is nullified.

Calculated absorption coefficients $\left(\alpha_{i f}\right)$ for transitions among different eigen states are shown in Figure: 4.6. The eigen states for square and stepped QWs were found to be at $0.02,0.08,0.167 \mathrm{eV}$ and $0.0393,0.124,0.186 \mathrm{eV}$ respectively. The absorption spectrum in Figure:4.6 confirms the breaking of selection rule in stepped QW, as it shows the dominance of $|1\rangle \rightarrow|3\rangle$ transition in terms of both $\alpha_{i f}$ and $\Gamma_{o p}$. For the square QW, however, $\alpha$ for $|1\rangle \rightarrow|3\rangle$ transition is several orders of magnitude smaller than that of $|2\rangle$ $\rightarrow|3\rangle$, which is consistent with the selection rule. Therefore, in terms of total broadening, we see $\Gamma_{|1\rangle}<\Gamma_{|2\rangle}$ for square QW. In case of stepped QW, it is other way around, i.e. $\Gamma_{|2\rangle}$ $<\Gamma_{|1\rangle}$, as selection rule forbidden $|1\rangle \rightarrow|3\rangle$ transition probability outweighs others.

Figure: 4.7 shows $\Gamma_{\mathrm{op}} / \Gamma_{\text {tun }}$, along with absorption coefficient as a function of Indium concentration. With the increase in In concentration (for a fixed $15 \% \mathrm{Al}$ ), step height increases. This increases the asymmetry of the QW and as a result optical coupling constant improves, resulting in increased absorption. At higher In concentration, however, the wavefunction of higher eigen state leaks out into the barrier. This reduces the overlapping between the wavefunctions and lower the amount of coupling and hence absorption. 


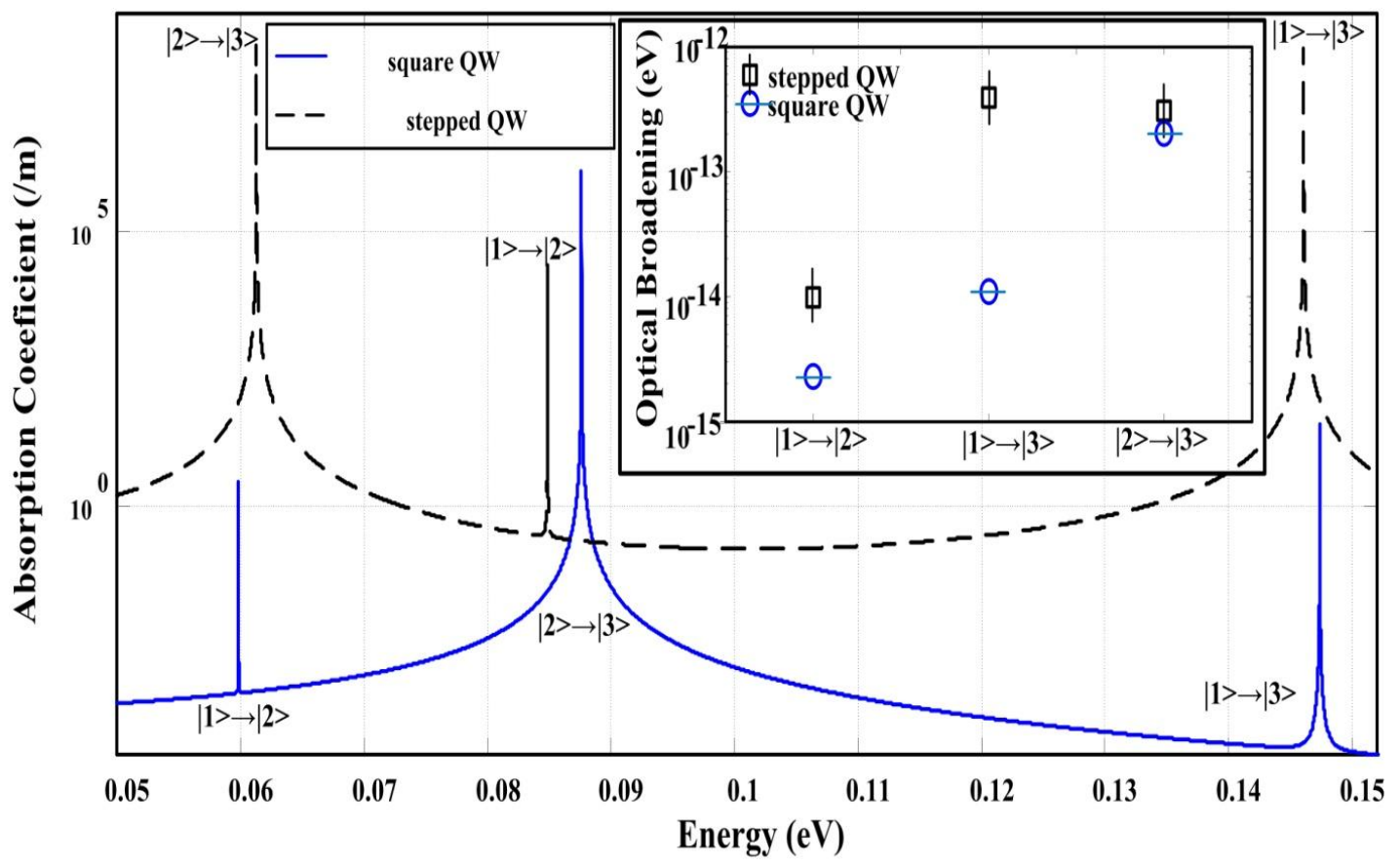

Figure 4.6: $\alpha_{i f}$ for a $30 \mathrm{~nm}-6 \mathrm{~nm}-6 \mathrm{~nm} \quad \mathrm{Al}_{x} \mathrm{Ga}_{1-\mathrm{x}} \mathrm{As} / \mathrm{GaAs} / \mathrm{In}_{y} \mathrm{Ga}_{1-y} A s$ stepped QW and it's square QW counterpart. Inset shows the optical broadening for different transitions.

For certain asymmetric structures like those having gradually doped well region, overlapping integral of $\psi_{f}$ and $\psi_{i}$, required to calculate normal incidence absorption, is proportional to the ratio of overlapping integral of $\psi_{f}$ and gradient of $\psi_{i}$, and the energy difference between the states [24]. Therefore, other things being equal, absorption of TE polarized light is expected to increase with the decrease of energy spacing between the two concerned states, or in other words for far infrared radiation. 


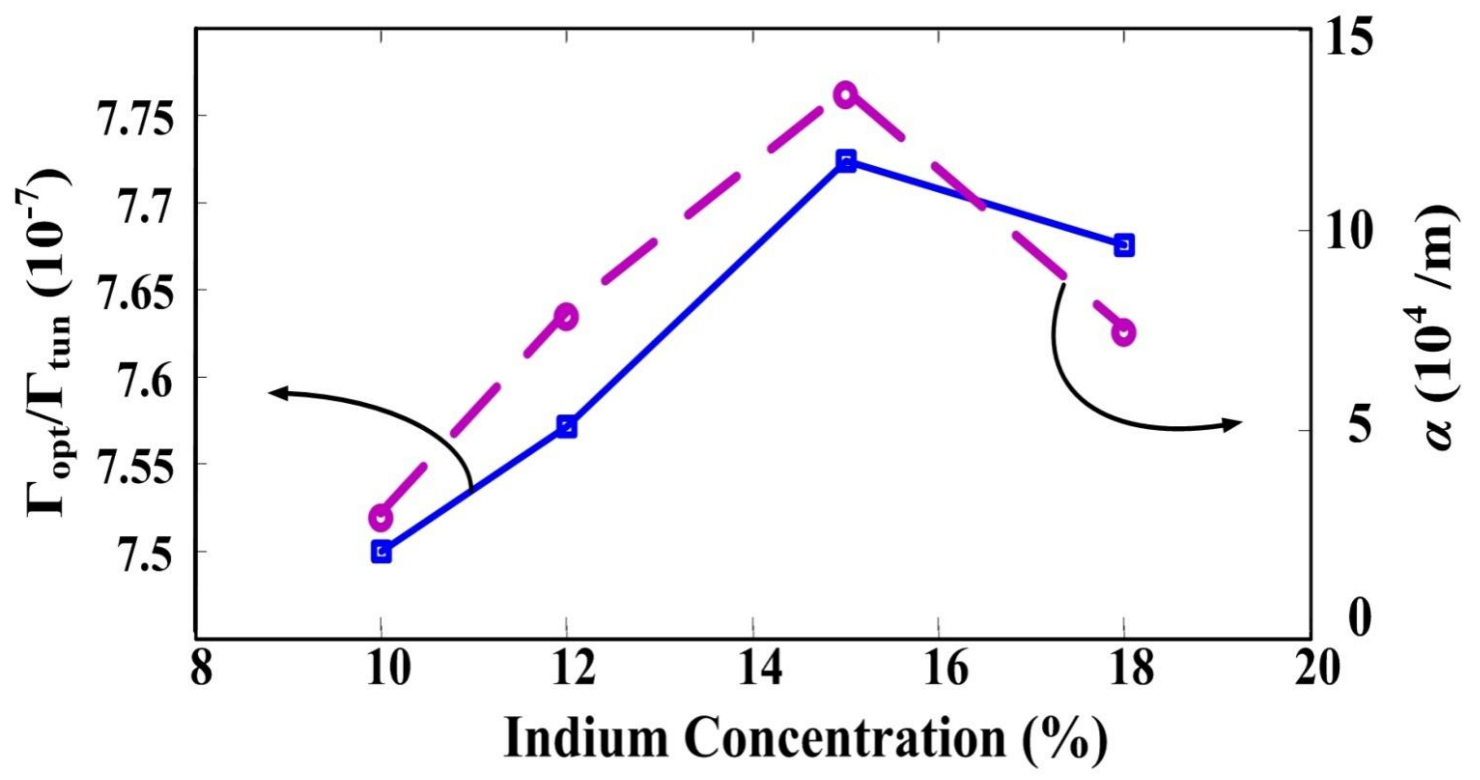

Figure: $4.7 \Gamma o p / \Gamma_{\text {tun }}$ and $\alpha$ calculated as a function of Indium concentration

\subsection{Simulation Setup for a Stepped Quantum Well}

Due to the asymmetry of the well, it is expected that the dipole moment of transition from one energy state to another increases significantly thus improving the optical transition rate. The deeper well is doped to increase the number of transition and thus increasing photocurrent. The proposed $\mathrm{AlGaAs} / \mathrm{In}_{\mathrm{x}} \mathrm{Ga}_{(1-\mathrm{x})} \mathrm{As} / \mathrm{GaAs} / \mathrm{AlGaAs}$ structure has been characterized using ATLAS. ATLAS is an advanced two-dimensional device simulator that incorporates both drift-diffusion and energy balance transport equations. A large selection of physical models is available which include surface/bulk mobility, recombination, impact ionization and tunneling models. Two major models part of this simulation are simulation of quantum mechanical effect and optoelectronic effect.

Quantum effect simulation: In order to simulate the device with quantum effects such as energy quantization and confinement, ATLAS exploits the solution of Schrodinger's 
equation along with the fundamental device equations. The solution of Schrodinger's equation provides a "quantized" description of the density of states in the presence of quantum mechanical confining potential variations. Among five quantum models available, Self-Consistent Schrodinger-Poisson model has been used. With this model, one dimensional Schrodinger equation is solved along $y$ mesh, for Eigen-energies and eigen functions using Fermi-Dirac statistics. Once the Schrodinger equation solution is obtained, carrier concentrations are calculated and are substituted into the Poisson's equation. The potential derived from solution of Poisson's equation is substituted back into Schrodinger's equation. This solution process (alternating between Schrodinger's and Poisson's equation) is continued until convergence is reached and a selfconsistent solution of Schrodinger's and Poisson's equation is obtained.

Following syntax has been used with model and output statement:

model schrodinger eigen $=\mathrm{n}$ fixed.fermi

output cond.band valence. band eigen $=\mathrm{n}$

Here fixed.fermi is used assuming Quasi-Fermi level is uniform across $\mathrm{Y}$ slice and is calculated to match the classical and quantum mechanical sheet charge; eigen=n solves for $\mathrm{n}$ eigenstates and schrodinger activates the model.

Optoelectronic effect simulation: LUMINOUS has been used to simulate the optoelectronic behavior of the quantum well structure. LUMINOUS is an advanced device simulator specially designed to model light absorption and photogeneration in non-planar semiconductor devices. Exact solutions for general optical sources are obtained using geometric ray tracing. This feature enables Luminous to account for 
arbitrary topologies, internal and external reflections and refractions, polarization dependencies and dispersion. Luminous also allows optical transfer matrix method analysis for coherence effects in layered devices. The beam propagation method may be used to simulate coherence effects and diffraction. LUMINOUS calculates optical intensity profiles within the semiconductor device, and converts these profiles into photogeneration rates in the device simulators. LUMINOUS is fully integrated within ATLAS with a seamless link to S-Pisces and Blaze device simulators, and other ATLAS device technology modules.

Optoelectronic device simulation with LUMINOUS is split into two distinct models that are calculated simultaneously at each DC bias point or transient time-step:

1. Optical ray trace using real component of refractive index to calculate the optical intensity at each grid point.

2. Absorption or photogeneration model using the imaginary component of refractive index to calculate a new carrier concentration at each grid point.

This is followed by an electrical simulation to calculate terminal currents. An optical beam is modeled as a collimated source using the BEAM statement. The origin of the beam is defined by parameters:

- X.ORIGIN and Y.ORIGIN.

- The ANGLE parameter specifies the direction of propagation of the beam relative to the $\mathrm{x}$-axis. ANGLE=90 is vertical illumination from the top. 
Results and Discussions: Following figures shows the quantum and optoelectronic results obtained for AlGaAs/InGaAs/GaAs structure. Figure 4.8 shows the conduction energy band profile with an applied voltage of 0.2 volt. It clearly demonstrates the formation of a stepped quantum well, InGaAs layer being the well and GaAs layer being the step. In this simulation both the layers are $4 \mathrm{~nm}$ thick. The well-step width ratio can be exploited to tune the wavefunctions and bound state energies to tailor the detection wavelength.

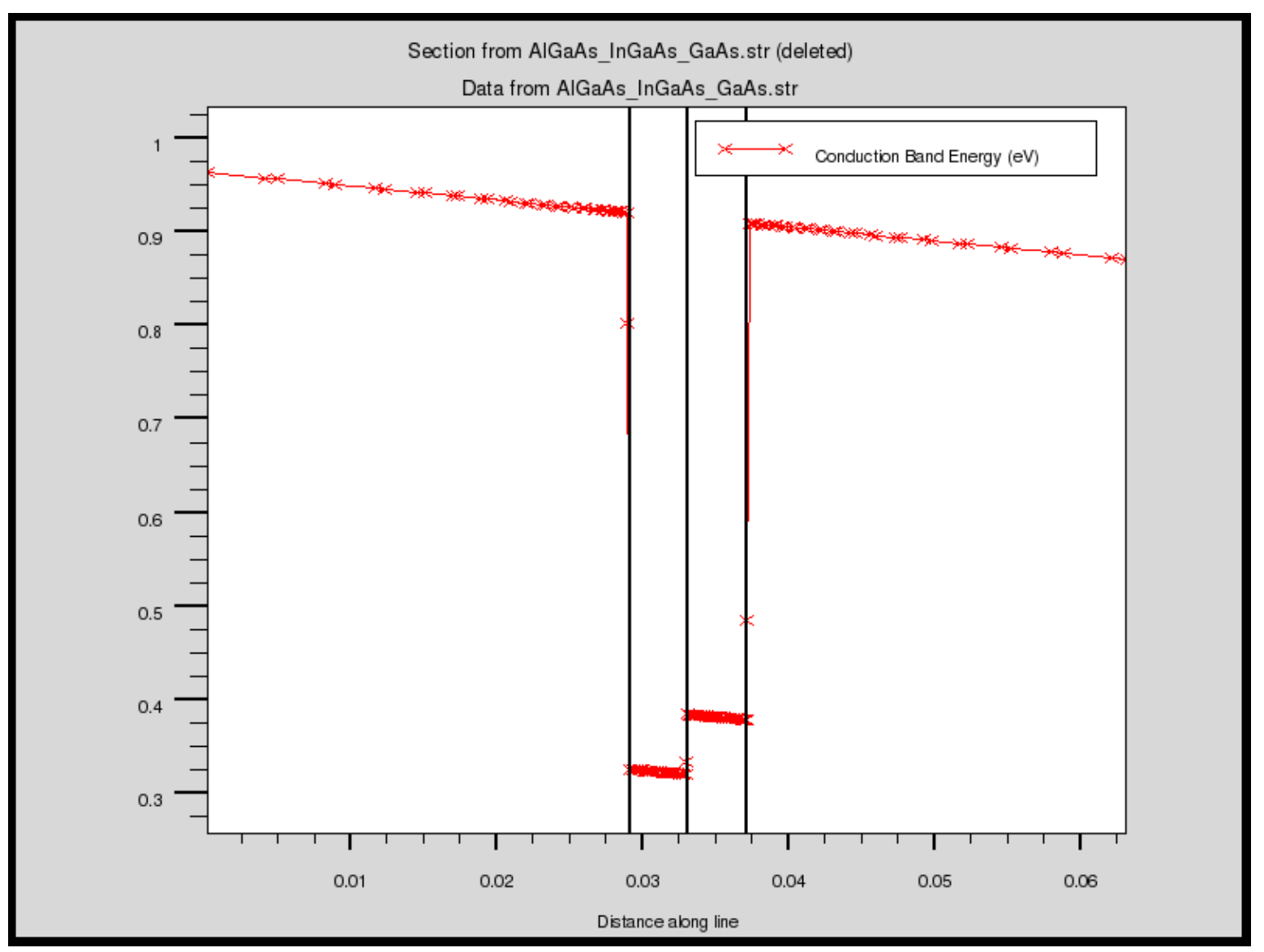

Figure 4.8: Conduction band profile of $\mathrm{AIGaAs} / \mathrm{ln}_{\mathrm{x}} \mathrm{Ga}_{(1-\mathrm{x})} \mathrm{As} / \mathrm{GaAs} / \mathrm{AIGaAs}$ structure 


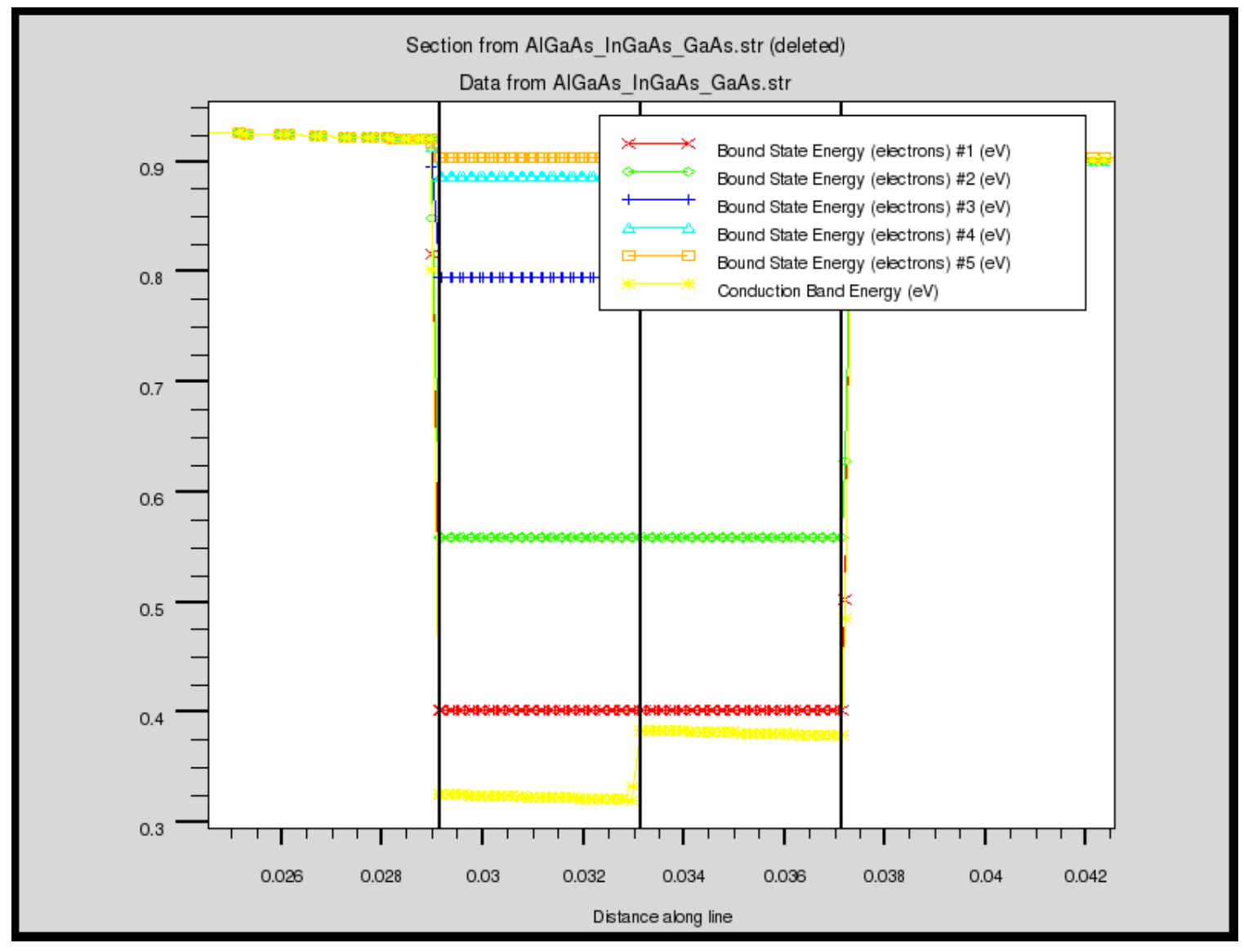

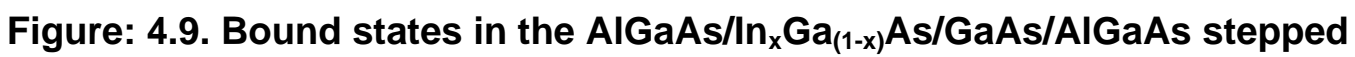
quantum well

Figure 4.9 shows first five bound states in the stepped quantum well. For this given structure the ground state is not confined within the well, but is rather spreaded over the step as well. The energy separation between first and $2^{\text {nd }}, 3$ rd, fourth, fifth states are approximately $0.16,0.4,0.48$ and $0.5 \mathrm{eV}$ respectively. These correspond to approximately $7.75 \mu \mathrm{m}, 3.1 \mu \mathrm{m}, 2.58 \mu \mathrm{m}$ and $2.48 \mu \mathrm{m}$ respectively. As expected the separation between adjacent energy states diminishes as one move up in the energy scale. 


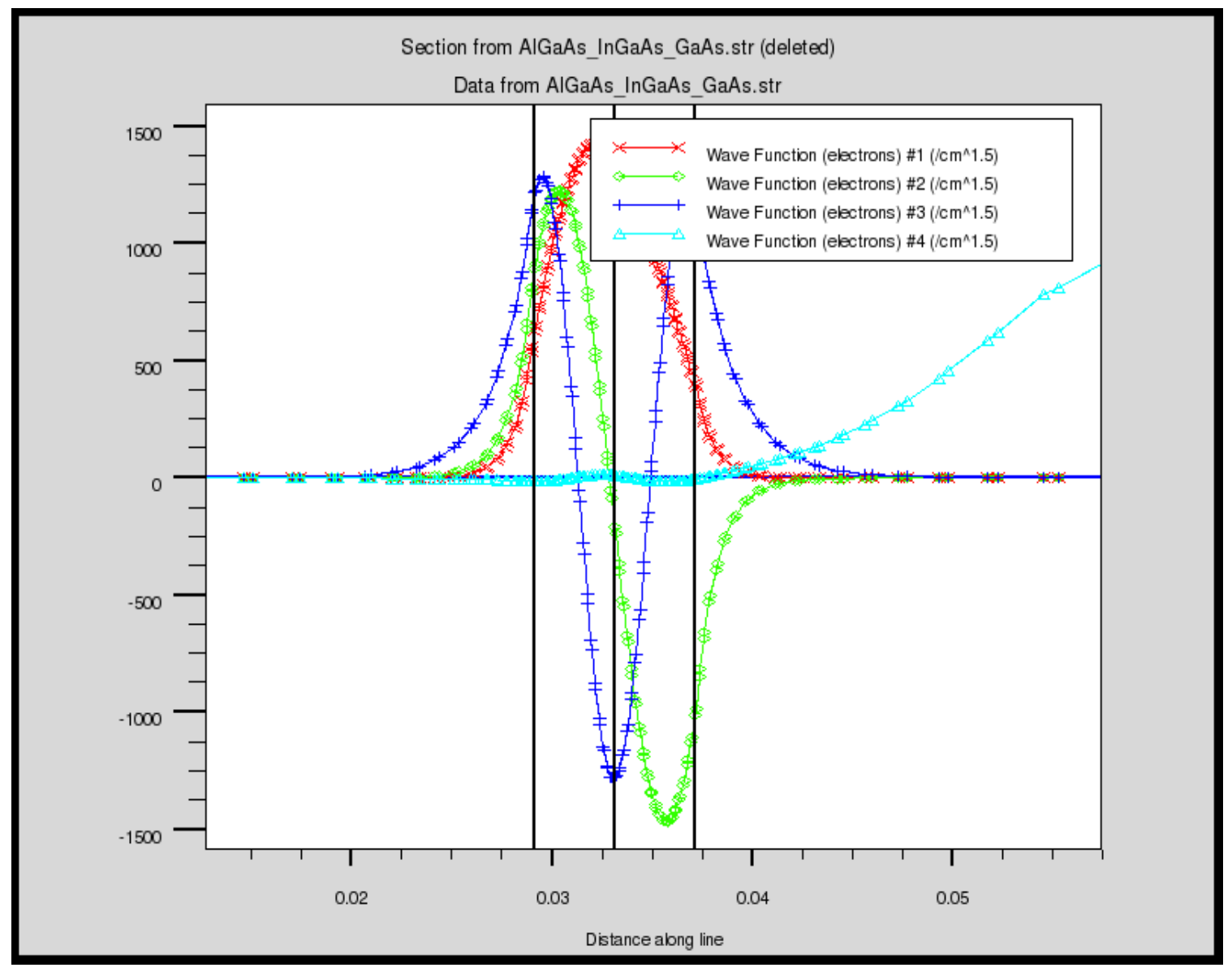

Figure: 4.10 Electron wavefunctions of the bound states.

The electron wavefunctions for the bound energy states are shown in Figure 4.10. The peak of the ground state is found to be in the well (InGaAs layer). This peak gets shifted more into the well layer if the In mole fraction is increased to increase the depth of the well. The major lobe of the wavefunction of the first excited state is found to be in the step (GaAs) layer. Since the third excited state is close to the continuum, the asymmetric nature of the quantum well almost has no impact on its shape and hence it is symmetrical. The fourth excited state is almost at the continuum and therefore shows extreme deformation in the direction of applied electric field. 


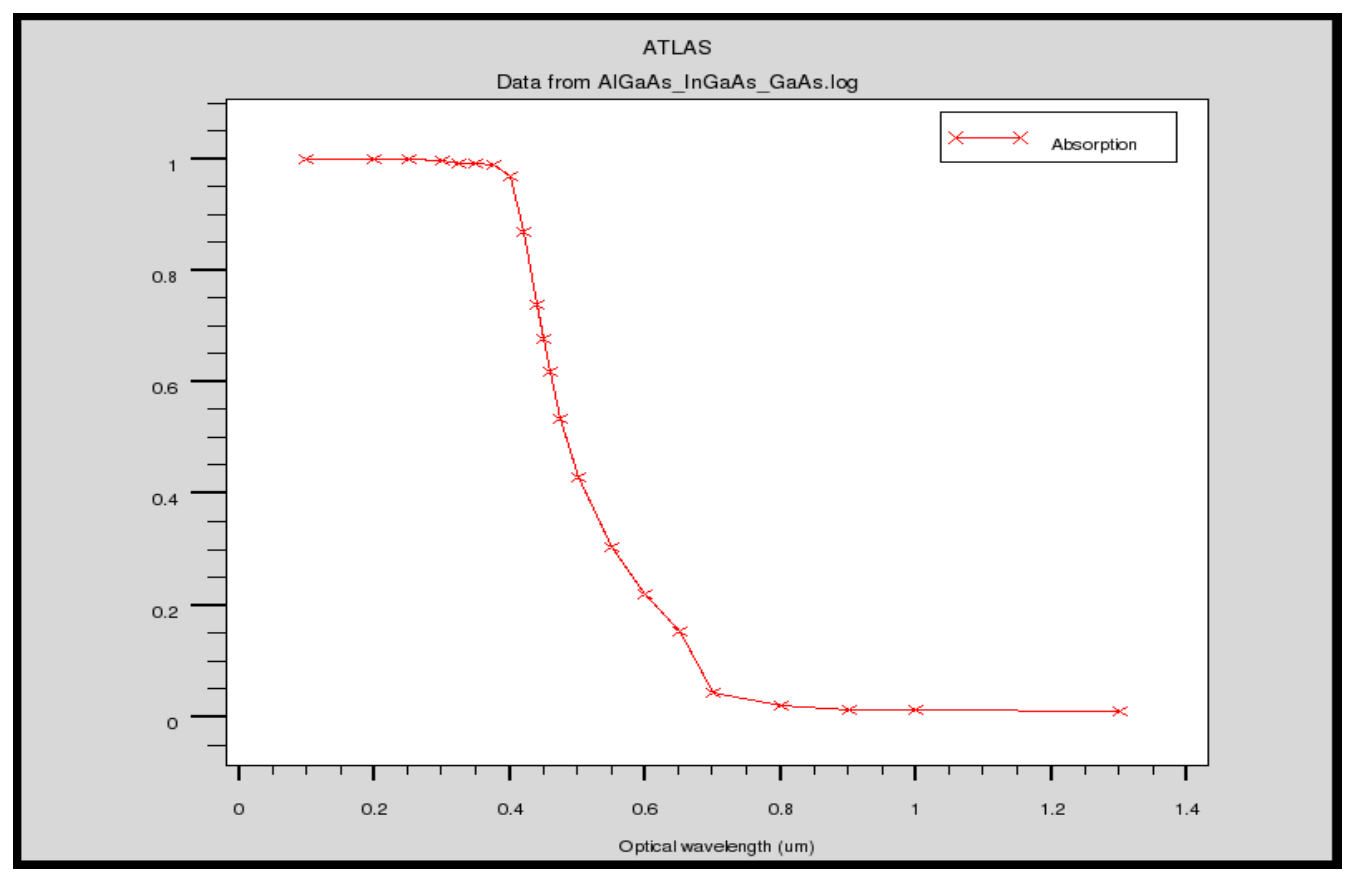

Figure 4.11: Absorption in the structure

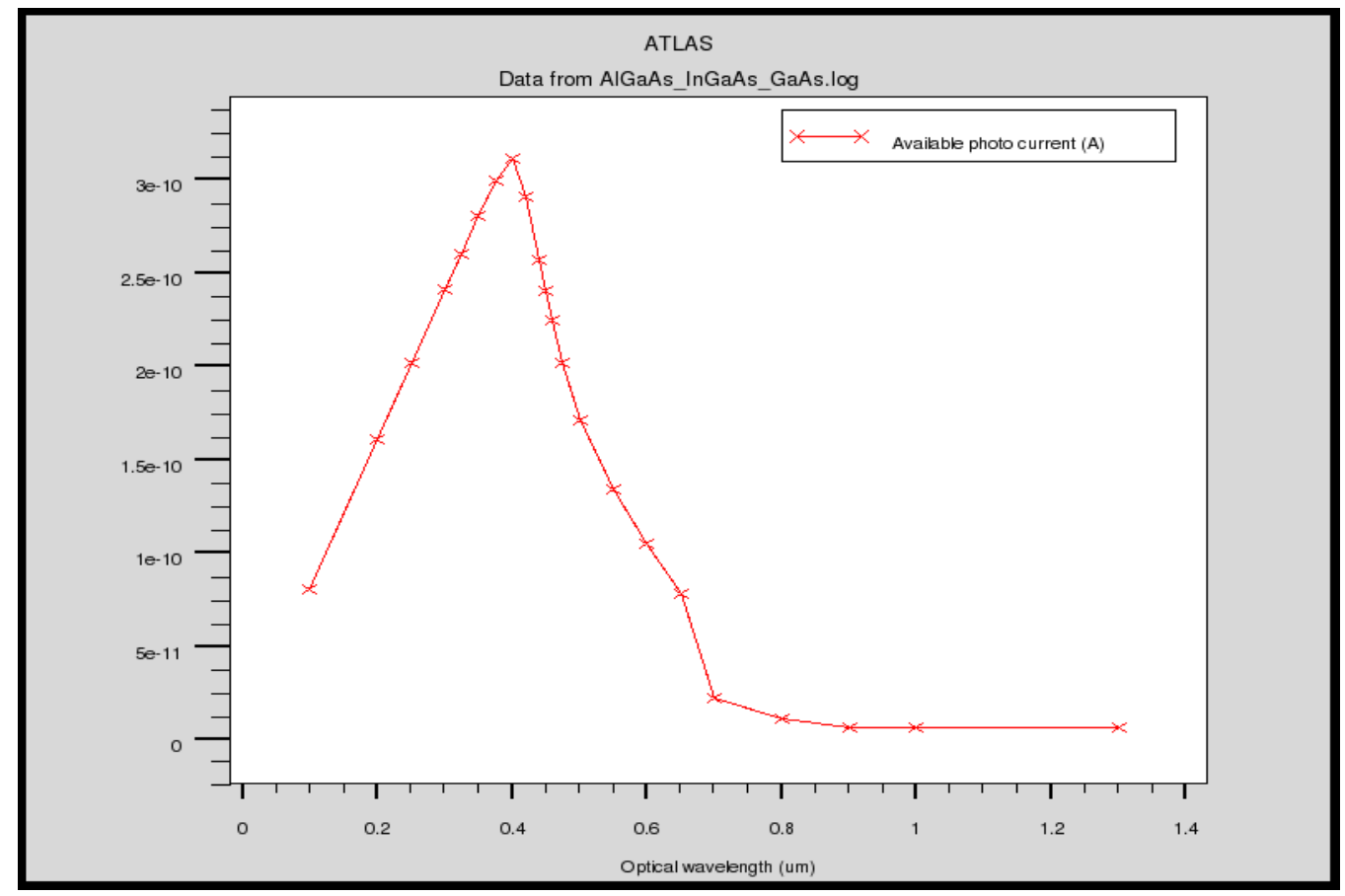

Figure 4.12 Spectral response of the structure 
Figure 4.11 and Figure 4.12 show the absorption and spectral response of the structure under consideration. The available photo-current is a good measure of the detection profile of the device. The peak is found to be around $0.4 \mu \mathrm{m}$. This implies that the detection has been mainly due to inter-band transition, rather than intra-band transition.

\subsection{Effect of Step Width on Absorption}

Investigation on the dimensions of the quantum well reveals that absorption peaks shift to the left as the width of the GaAs step is increased. This is because the energy of the first excited state, which is above the conduction band edge of the step, shifts downward with increasing step width. However, since the ground state is bounded in the well, its energy is relatively insensitive to the changes of the step width. As a result, the ground state to first excited state transition energy decreases and the spectra shift towards the lower energy region.

The probability densities of the both the states are shown in Figure: 4.13, for various step widths of the GaAs layer. The vertical line in Figure: 4.13 marks the abrupt interface between the barrier and the step. At a step width of $15 \mathrm{~A}^{\circ}$, the first excited state is quasi-bound and its energy is slightly below the edge of the conduction band of the barrier. The energetic quasi-bound wavefunction still possesses considerable magnitude in the barrier region, and thus appears rather symmetrical. As the step width is increased, the energy of the first excited state is lowered and the wavefunction are found to get more tightly bound. The coupling constant between the ground state and the first excited state wavefunctions increases initially due to the rapid increase of the probability density of the first excited state wavefunction in the step and the well regions. However, as the step 
width is increased to about $30 \mathrm{~A}^{\circ}$, the smaller secondary lobe of the first excited state wavefunction that resides in the well reaches a maximum and then starts to decrease. At the same time, the peak of the ground state wavefunction continues to move away from the step region and decreases in magnitude while decaying more slowly into the step, as a result of which the gradient of the wavefunction decreases. The field coupling of the $\psi_{1}$ and $\psi_{2}$ wavefunctions reaches its peak and saturates. Although the magnitude of the main lobe of the bounded first excited state residing in the step region increases with the step width, that of the secondary lobe residing in the well decreases, thus reducing the coupling with the ground state in the well. As a result, the absorption strength decreases for widths of the GaAs step greater than $30 \mathrm{~A}^{\circ}$.

\subsection{Impact of Strain}

Quantum well infrared photodetectors were first introduced using $\mathrm{GaAs} / \mathrm{Al}_{x} \mathrm{Ga}_{1-}$. ${ }_{x}$ As lattice-matched multiple quantum wells. By varying the Al molar ratio $x$ in the barriers, a wide range of intersubband absorption wavelengths can be obtained. However, a high $\mathrm{Al}$ molar ratio is less desirable in detector applications. Besides the presence of higher defect density, the energy crossing of the $\Gamma$ and $X$ valleys at $x>0.45$ reduces the thermal activation energy of the ground state electrons. The correspondingly higher dark current reduces the detector sensitivity in this material. Therefore the material characteristics for a mid-wavelength QWIP, which requires a large value of $x$, still need improvement. In order to increase the band offset between the well and the barrier without increasing $x, \operatorname{In}_{y} \mathrm{Ga}_{1-y} \mathrm{As}$ was introduced as the well material to reduce the conduction band offset. Due to the concern of lattice-mismatch between InGaAs and 
$\mathrm{AlGaAs}$ in the early attempts, a small indium molar ratio $y$ was adopted, such as that of 0.16. In this section the impact of strain is investigated.
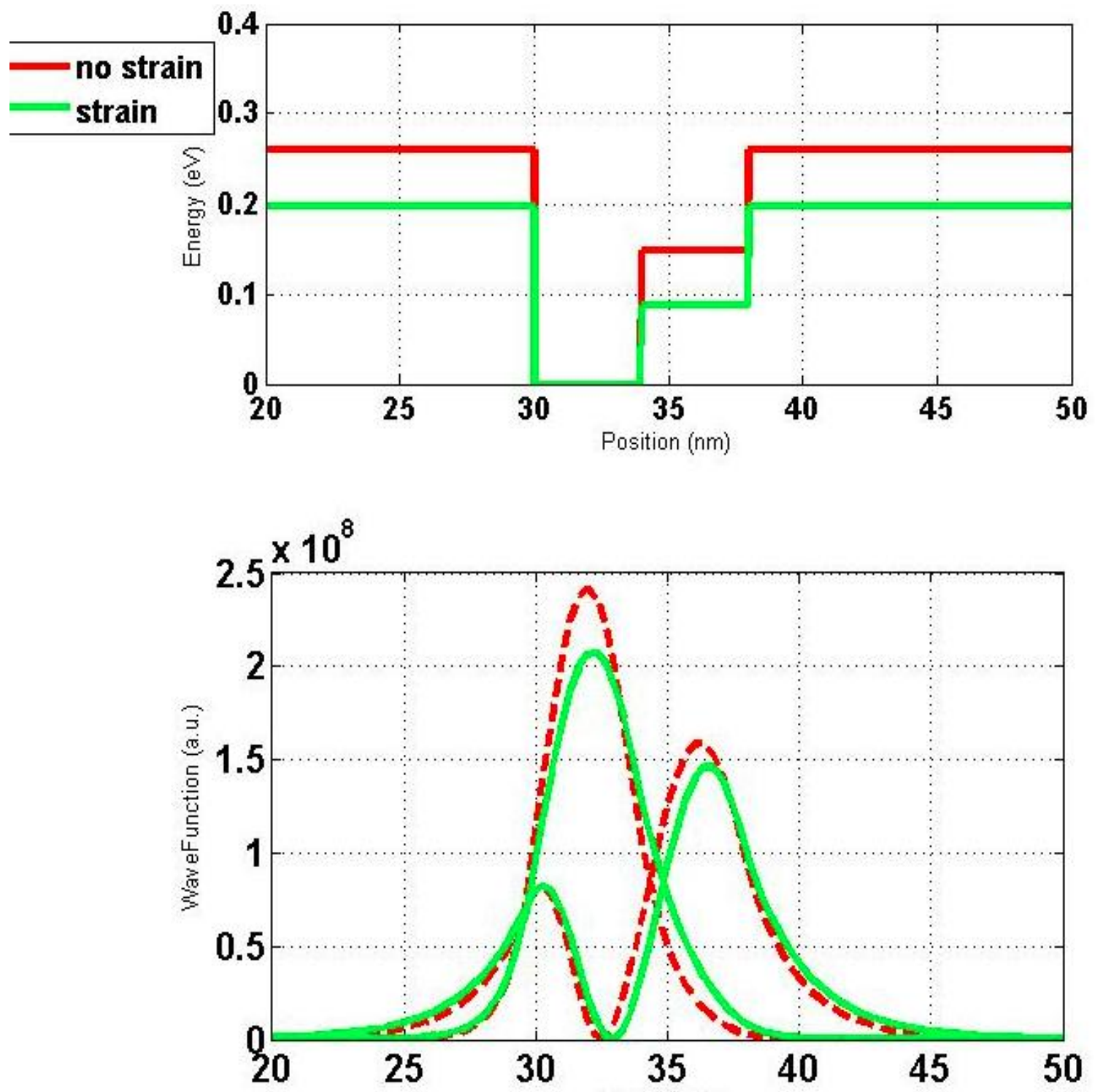

Figure: 4.13. Effect of strain on conduction band profile and wavefunctions

As has been detailed in chapter 3 the strain is developed due to juxtaposing different layers of material effect the bandgap of any given material. The unstrained band gap of the $n$th layer in the unit of $\mathrm{meV}$ is 


$$
E_{g, n}^{u}=1424+1186 x_{n}+370 x_{n}^{2}-1499 y_{n}+429 y_{n}^{2},
$$

where $x_{n}$ and $y_{n}$ are the $\mathrm{Al}$ and In molar ratios in the $n$th layer, respectively. Because of strain, the values of band gaps are modified. The modified band gap for $y_{n}>0$ is

$$
E_{g, n}^{s}=E_{g, n}^{u}+2 a\left(1-\frac{c_{12}}{c_{11}}\right) \varepsilon_{11}
$$

where $\quad a=-8700+2700 y_{n} \mathrm{meV}$ is the deformation potential, $c_{12} / c_{11}=\left(5.32-0.79 y_{n}\right) /\left(11.81-3.48 y_{n}\right)$ is the ratio of the elastic constants, and $\varepsilon_{11}=-0.405 y_{n} /\left(5.66533-0.405 y_{n}\right)$ is the hydrostatic component of the strain induced by the adjacent GaAs/AlGaAs material layers. The band offset $\Delta E_{n}$ is taken as

$$
\Delta E_{n}=0.60\left(E_{g, n}^{u}-1424\right) \quad \text { if } x_{n} \geq 0 \text { and is } 0.63\left(E_{g, n}^{u}-1424\right) \quad \text { if } y_{n}>0 \text {. }
$$

The effective mass at the conduction band edge is given by,

$$
m_{n}^{*}=0.0665\left(1+1.256 x_{n}-0.579 y_{n}\right)
$$

The resulting effective mass $m_{n} *(E)$ can be expressed in the form of

$$
m_{n}^{*}(E)=m_{n}^{*}\left(1+\frac{E-\Delta E_{n}}{E_{g, n}}\right)
$$

Figure 4.14 shows the impact of strain of the conduction band profile and wavefunction. As can be seen that due to strain being considered, the well depth is reduced. This also reduces the well to step height ratio. As a result the strained wavefunction is seen to be slightly more spreaded over the step.

Figure 4.14 demonstrates the impact of strain on the absorption peak. It is found that the absorption peak shifts from $\sim 0.14 \mathrm{eV}$ to $0.105 \mathrm{eV}$ due to stain being considered. 
That means due to strain, the quantum well becomes more responsive to far infrared region.

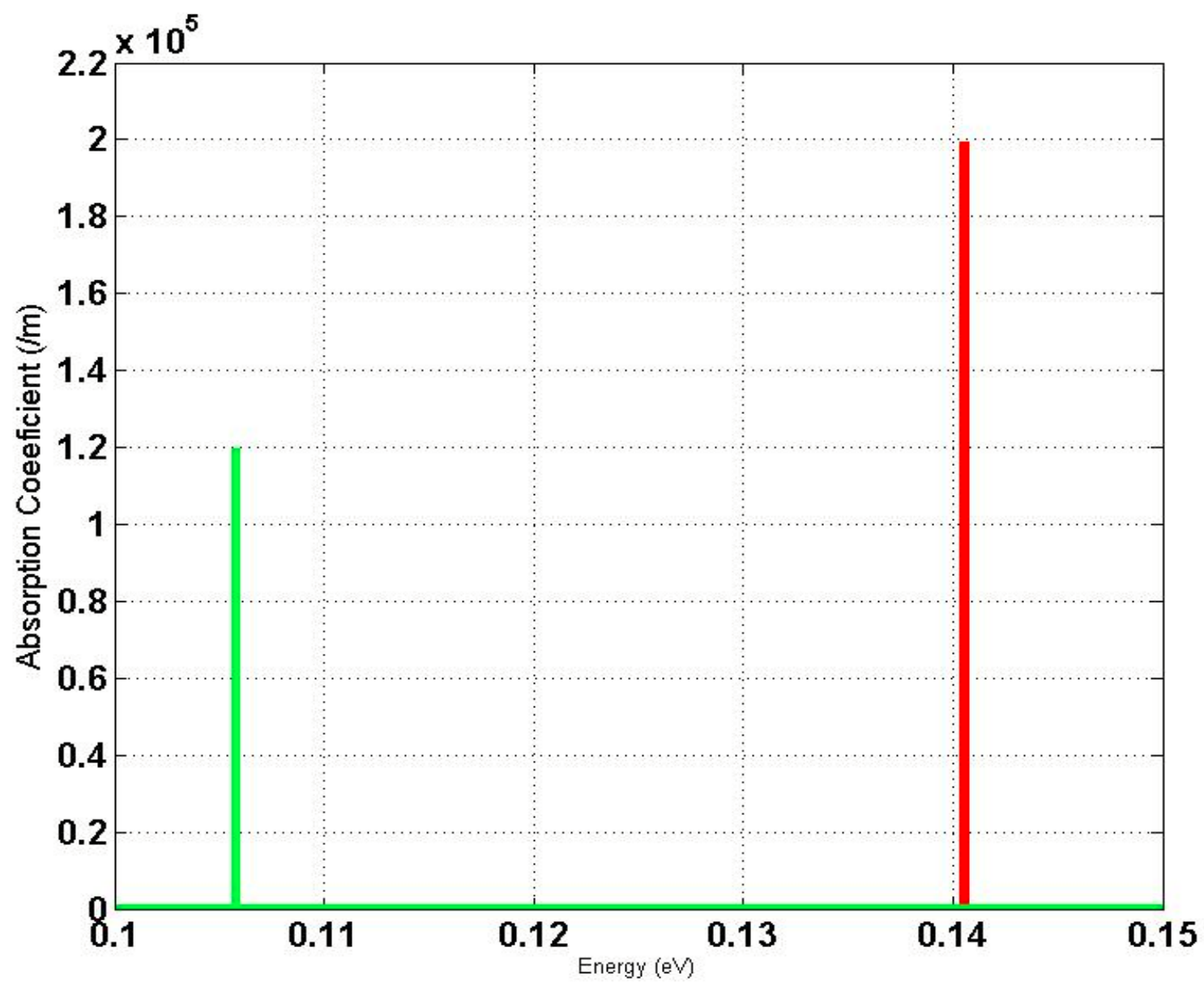

Figure 4.14: Impact of strain on absorption spectrum.

\subsection{Multiple Wavelength Detection and Voltage Tunability}

By using multiple quantum wells, instead of a single one, one can achieve detection at more than one wavelength. To illustrate and quantify the idea, presented in this section is the results for AlGaAs/InGaAs based multiple well structures. In this case, 
one is a normal rectangular well, whereas the other is a step well as has been used in this work.
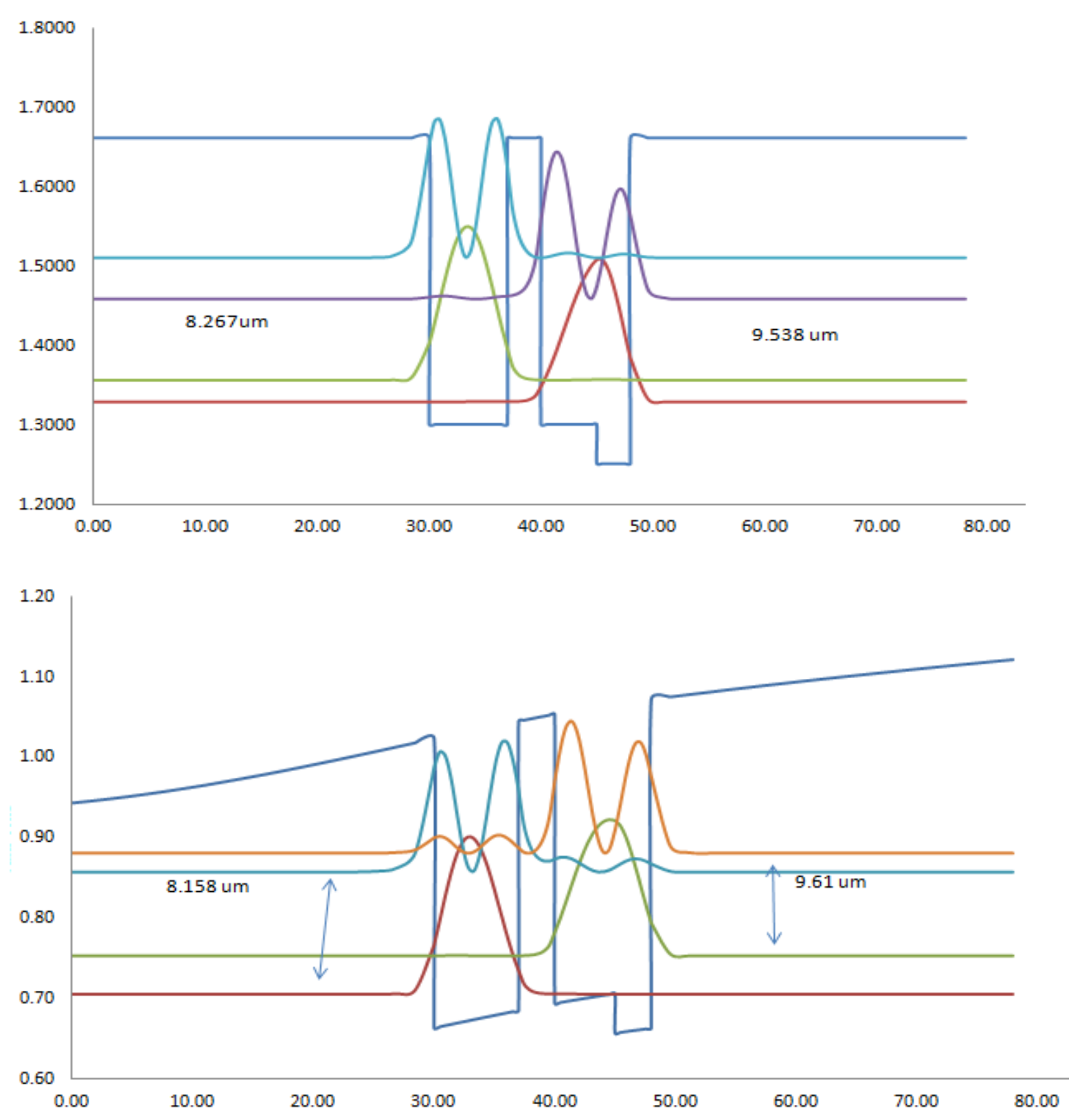

Figure: 4.15 Multiple quantum well structures demonstrating multiple wavelength detection. Top figure is at zero bias, whereas the bottom one is biased at $1 \mathrm{~V}$. 


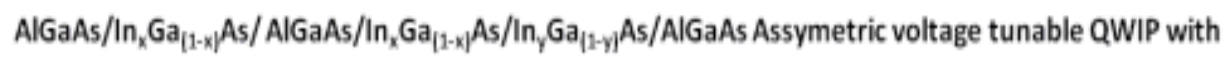
$x=1.5 \%, y=8 \%$ and $40 \% \mathrm{Al}$ :

( $30 \mathrm{~nm} / 7 \mathrm{~nm} / 3 \mathrm{~nm} / 5 \mathrm{~nm} / 4 \mathrm{~nm} / 30 \mathrm{~nm})$ with $1 V$ bias

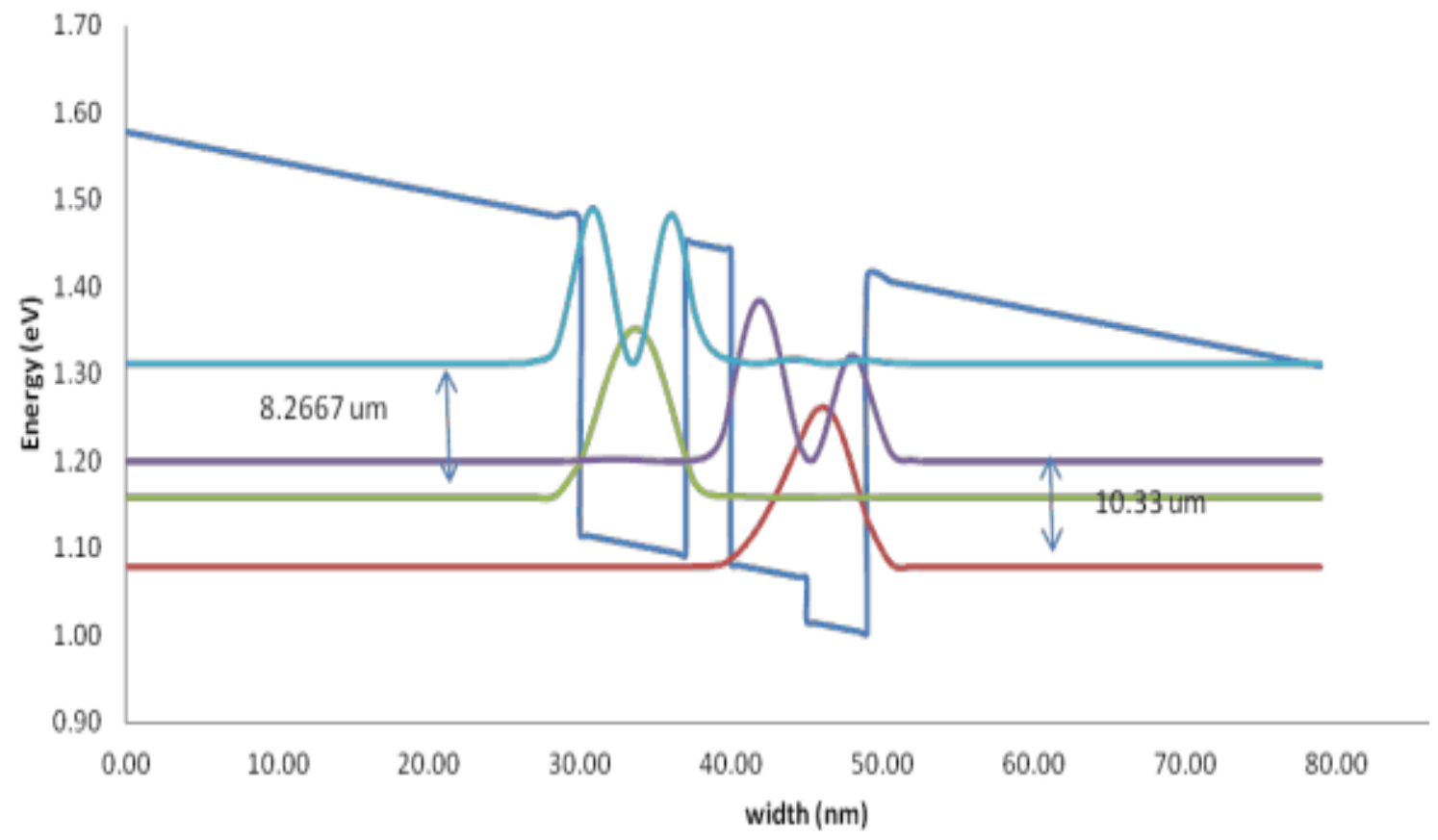

Figure: 4.16 Increased wavelength separation achieved through increased stepwidth and reversed applied voltage.

Figures 4.15 and 4.16 show that by applying voltage, the separation among the ground state and first excited state and hence the detection wavelengths can be increased. For example Figure: 4.15 shows that $1 \mathrm{~V}$ can increase the wavelength difference from $1.27 \mu \mathrm{m}$ to $1.46 \mu \mathrm{m}$. On the other hand, Figure: 4.16 shows that by increasing the step width the wavelength difference can be increased to $2.1 \mu \mathrm{m}$. This suggests that both voltage and step width can be used as variables to enlarge the separation between the detection wavelengths. 


\subsection{Conclusion}

The quantitative analysis of asymmetric quantum wells based on AlGaAs/InGaAs/GaAs structures has been presented in this chapter. In case of AlGaAs/InGaAs/GaAs structure, GaAs layer forms the step, whereas InGaAs forms the well. The composition of In can be varied to tune the step and well heights.

The investigation on optical absorption in asymmetric quantum well photodetectors has been presented in this chapter. The analysis has been based on nonequilibrium Green's function and quantum mechanical wave impedance concept. Unlike previously reported works that assume broadening of energy states due to intersubband transition for calculating absorption coefficient, the presented technique quantifies broadenings resulting from both optical absorption and tunneling. Results have been presented for $\mathrm{Al}_{x} \mathrm{Ga}_{1-x} \mathrm{As} / \mathrm{In}_{y} \mathrm{Ga}_{1-y} \mathrm{As} / \mathrm{GaAs}$ system which confirms that asymmetry in a QW breaks the symmetry or parity of the bound state wavefunctions and hence facilitates transition among certain energy states, which are otherwise forbidden by the selection rule. Assuming TM polarization of light, it has been demonstrated that stepped QW structures can in fact have improved absorption coefficient due to this symmetry breaking. The approach presented here can be extended to calculate the response of any arbitrary asymmetric well to both TE and TM polarization.

It is worth mentioning here that Indium concentration was varied to change the step height and thus to increase the "asymmetry" to clarify how symmetry breaking improves transition among certain states. Variation of $\mathrm{Al}$ concentration will change the well depth and therefore eigen energies and coupling among the states. But as that may 
not have any strong/direct impact on breaking of wavefunction symmetry, study involving variation of $\mathrm{Al}$ concentration was not carried out. 


\title{
CHAPTER 5
}

\section{Summary and Concluding Remarks}

\author{
Absorption of photons having energy corresponding to infrared radiation excites \\ electrons in the ground state of quantum wells in the conduction band of semiconductor \\ heterostructures to empty higher energy excited states. These photoexcited carriers can be \\ made to escape the quantum well and flow through an external circuit under applied \\ electric field. Since the energy concerned in this case is within infrared region, intra band \\ or inter subband transition within conduction band is particularly suitable, compared to \\ band to band or inter band transition. Quantum-well infrared photodetectors (QWIPs) are \\ operated based on this mechanism. Typically the detection wavelength of QWIPs spans \\ from 2 to $20 \mu \mathrm{m}$ or even longer. As have already discussed, these detectors have many \\ important applications such as remote temperature sensing, target identification and \\ discrimination, and chemical analysis. In this dissertation, we have investigated quantum \\ well structures, with a particular emphasis on the asymmetric ones that can be used as \\ QWIPs.
}




\subsection{Asymmetric Quantum Wells and Material Choice}

When QWIPs were first introduced, rectangular or square quantum wells were considered as the obvious choice. Due to matured growth technology, $\mathrm{GaAs} / \mathrm{Al}_{x} \mathrm{Ga}_{1-x} \mathrm{As}$ lattice-matched multiple quantum wells were extensively studied. It was found that, by varying the $\mathrm{Al}$ molar ratio $x$ in the barriers, a wide range of intersubband absorption wavelengths can be obtained. Also InGaAs/AlGaAs quantum well structures have been shown to be versatile for infrared detection. By changing the material composition, one can tune the detection wavelength from 2 to $35 \mathrm{~mm}$ and beyond. The most important features of these QWIPs involving III-V semiconductors that make them superior to MCT based ones are the chemical stability of the materials involved and their well developed growth-process technology. QWIPs have become dominant in the area of long wavelength IR focal plane array (FPA) applications in the last few years in terms of array size, uniformity, yield and cost of the systems. The other properties of QWIP, such as high impedance, fast response time, long integration time and low power consumption are well tailored to the requirements for the fabrication of large FPAs.

However, it was later realized that, as electrons in these III-V semiconductor occupy the $\Gamma$ valley, the associated wavefunctions posses certain symmetry and parity. This leads to a "selection rule" due to which transition among certain energy states are forbidden. This phenomenon limits the overall absorption capability of the structure. Therefore effort has been made to eliminate the symmetry or parity of the wavefunction. Simpler rectangular or square wells are being replaced by quantum wells of different shape. These asymmetric quantum wells allow transition among all the eigen staes and hence can improve the overall detection efficiency. The asymmetry in the quantum well 
can be introduced in several forms. For example applied bias can make the bottom of the conduction band slanted and thus can make the wavefunction skewed to one direction. Similar effect can be brought in by using gradual doping. However, the elegant approach to introduce asymmetry is to use stepped quantum well. Steps are made by deploying layers of different materials to form the well region. Use of stepped quantum well formed through AlGaAs/ $\operatorname{In}_{x} \mathrm{Ga}_{(1-\mathrm{x})} \mathrm{As} / \mathrm{In}_{\mathrm{y}} \mathrm{Ga}_{(1-\mathrm{y})}$ As/AlGaAs structure, which has been the main focus of this work, can further improve the wavelength tunability of the QWIP. The high electron mobility and hence drift velocity in $\operatorname{InGaAs}\left(>5 \times 10^{6} \mathrm{~cm} / \mathrm{s}\right)$, along with low carrier recapture lifetime, is expected to improve the detection speed of the device. Unlike GaAs/AlGaAs, the lattice mismatch at InGaAs/AlGaAs interface is likely to introduce strain in the structure, which, if carefully optimized through deploying appropriate thickness, can augment QWIPs performance improvement.

\subsection{Quantifying Optical Absorption and Carrier Transport}

A mesoscopic representation of the quantum processes that govern both vertical (absorption) and lateral transport (tunneling) of electrons in asymmetric QW structures, has been utilized to quantify the optical and transport mechanism in asymmetric quantum wells. In order to quantify the optical properties, which include both the absorption phenomena through photogeneration and carrier transport across the structure, a quantum mechanical model based on non-equilibrium Green's function, Fermi's golden rule and quantum wave impedance has been developed. The model can calculate the eigen energy states, and the associated wave functions of a given quantum well structure using NEGF and qauntum wave impedance concept. Fermi's Golden rule is then used to calculate the 
coupling coefficients among the states in illuminated condition to determine the absorption coefficient. The calculation model developed and used in this dissertation has two distinct advantages. The first obvious advantage is that it accurate, easily programmable and yet does not sacrifice any computational efficiency. Second, it facilitates quantifying the broadening of states resulting from both photon absorption and tunneling, which provides important insight for improving detection efficiency. Instead of being calculated and optimized through calculations, such broadening has been simply assumed in previously reported works. In order to calculate the carrier transport across the quantum well structure, the concept of quantum well impedance has been used. This methodology uses well developed microwave transmission line theory to calculate the tunneling or transmission coefficient as a function of electron energy. The tunneling coefficient is needed to calculate the current voltage characteristics under both illuminated and dark condition.

\subsection{Analyzing the Symmetry Breaking Through Asymmetric QW}

It has been discussed in detail in previous chapters that symmetry conditions impose a "selection rule" that diminishes the probability of electron transition among eigen states having definite parity. Furthermore it also forbids transition being induced by photons with no electric field component in the growth direction. As a consequence, photons with transverse electric (TE) polarization with respect to the growth direction cannot be absorbed in a rectangular or square QW structures. A number of ingenious techniques have been proposed to overcome this, including fabrication of grating structures on top contact, illumination through 45 degree facet, and a scheme to arrange 
multiple pass of photons with oblique incidence. However, these alternatives, although can improve the normal incidence to some extent, complicates the focal plane fabrication process, and induce cross talks among adjacent pixels.

The main motivation behind adopting asymmetric QW structures has been the breaking of wave function symmetries, despite the fact that such symmetry conditions does not strictly hold for real rectangular wells due to various reasons like surface roughness, truncation of periodic bulk structure, etc. Achievable through steps in quantum well, asymmetry "distorts" the bound state wave functions in a way that they no longer posses' definite parity. AlGaAs/GaAs/InGaAs stepped quantum well structure has been studied in this work to quantify the impact of steps in the quantum well region on coupling coefficients among different energy states. The absorption rate of photons with certain energy is directly proportional to the coupling coefficient between the energy stated that are separated by that similar amount of energy. This coupling coefficient, which is also referred to as oscillator strength, is increased considerably as wave function symmetry is broken. The question that is of enormous importance interest from the device viewpoint, is whether it is possible to design quantum well structures in which normal incident infrared radiation can be readily absorbed by transitions between conduction-band subband states. This thesis has attempted to demonstrate that asymmetric QW structures can absorb such radiation, whereas symmetric structures such as a rectangular or square QW will absorb only weakly. On the basis of the present approach, the "best" materials for infrared QW detectors will be those in which the effective mass of the conduction band electron shows the maximum variation with material composition. Similarly, heavily graded doping within the well region of the QW 
structure will increase the amount of the infrared absorption. It is worth mentioning here that an intense external electric fields of the appropriate orientation can produce the similar increase in absorption by creating the desired slope in the energy band profile. It has been demonstrated that one will need to design carefully the actual composition of the QW structure or in that sense, QW potential profile, to obtain an appreciable value for the overlap integral of the wavefunction of the concerned energy states. This increased overlapping integral increases the amount of absorption of normal incidence infrared radiation.

\subsection{Future Work}

This section provides a brief overview of what can be done further to improve the understanding of the intra subband absorption and hence better optimized the QWIP performance.

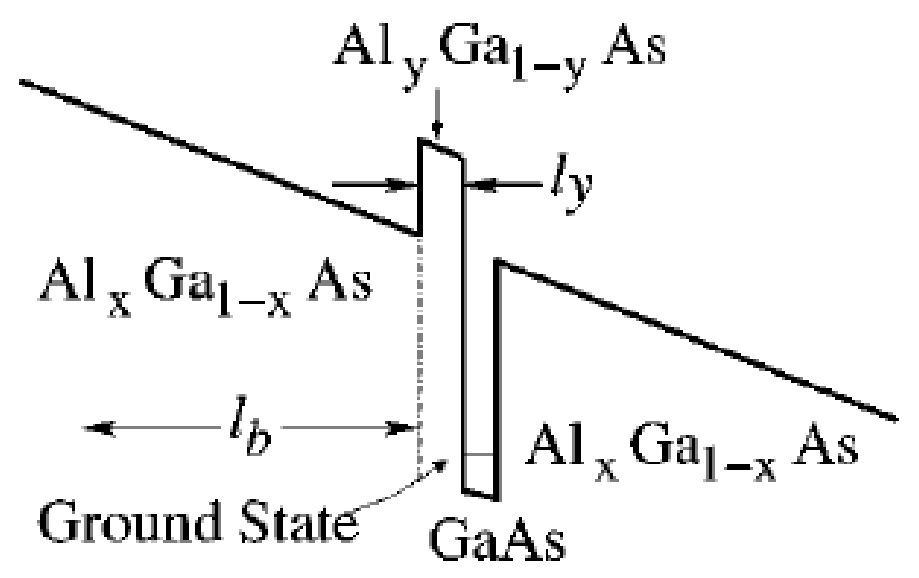

Figure: 5.1 Modified potential profile for dark current reduction 


\section{Reduction of dark current}

The improvement of responsively of a QWIP depends on lowering the dark current. Investigation, therefore needs to be carried out to reduce the dark current density. Figure:5.1 shows one such potential structure that can reduce the dark current. In this structure, the tunneling probability across the device is going to be considerably reduced due to the added barrier.

\section{Lateral Quantum confinement}

Quantum dots (QDs) have three dimensional electron confinement that leads to atomic-like energy levels. The selection rules that forbid normal incident absorption in QWs are relaxed in QDs. Therefore, quantum-dot infrared photodetectors (QDIPs) would exhibit intrinsic normal incidence absorption. Also, the oscillator strength of optical transitions are expected to be much larger than those in QWs. Compared to QWIPs, QDIPs are also expected to have a larger photoconductive gain because of the larger intersubband relaxation time, which results from reduced electron-phonon interaction in zero-dimensional systems . For these reasons, QDIPs have been the topic in intensive research for the past decade or so as they are attractive candidates for infrared imaging. QDIPs fabricated so far are based on self-assembled quantum dots that rely on the strain between lattice mismatched semiconductors. 


\section{REFERENCES:}

[1] K.K. Choi, S.V. Bandara, S.D. Gunapala, W.K. Liu and J. M. Fastenau, J. Appl. Phys., Vol. 91( 2), 551(2002).

[2] B. F. Levine, K. K. Choi, C. G. Bethea, J. Walker, and R. J. Malik, Appl. Phys. Lett. 50, 1092 (1987).

[3] Je' ro^ me Faist, Federico Capasso, Carlo Sirtori, Deborah L. Sivco, Albert L. Hutchinson and Alfred Y. Cho, Nature, Vol 387, 777(1997).

[4] IEE Proc. Optoelectron., Vol. 145(1), 21 (1988).

[5] C.W. Cheah, G. Karunasiri and L. S. Tan, Semicond. Sci. Technol. 17 (2002) 1028-1037

[6] A. Rogalski, J. Appl. Phys., 93(8), 4355 (2003)

[7] S. D. Gunapala and K. M. S. V. Bandara, in Thin Films, edited by M. H. Francombe and J. L. Vossen Academic Press, New York, 1995!, Vol. 21, p. 113.

[8] S. D. Gunapala and S. V. Bandara, in Handbook of Thin Devices, edited by M. H. Francombe $\sim$ Academic Press, San Diego, 2000!, Vol. 2, p. 63

[9] B. F. Levine, C. G. Bethea, G. Hasnain, J. Walker, and R. J. Malik, Appl. Phys. Lett. 53, 296 (1988).

[10] B. F. Levine, J. Appl. Phys. 74, R1 (1993)

[11] B. F. Levine, C. G. Bethea, G. Hasnain, J. Walker, and R. J. Malik, Appl. Phys. Lett. 53, 296, (1988).

[12] B. F. Levine, C. G. Bethea, K. K. Choi, J. Walker, and R. J. Malik, J. Appl. Phys. 64, 1591 (1988).

[13] B. F. Levine, C. G. Bethea, G. Hasnain, J. Walker, and R. J. Malik, Electron. L\&t. 24, 747 (1988).

[14] B. F. Levine, G. Hasnain, C. G. Bethea, and N. Chand, Appl. Phys. Lett. 54, 2704 (1989).

[15] B. F. Levine, C. G. Bethea, G. Hasnain, J. Walker, R. J. Malik, and J. M. Vandenberg, Phys. Rev. Lett. 63, 899 (1989).

[16] P. Helgesen, R. Sizmann, S. Lovold, and A. Paulsen, Proc. SPIE 1675 (1992)

[17] J. L. Pan, L. C. West, S. J. Walker, R. J. Malik, and J. F. Walker, Appl. Phys. Lett. 57, 366 (1990)

[18] K. K. Choi, B. F. Levine, C. G. Bethea, J. Walker, and R. J. Malik, Appl. Phys. Lett. 52, 88).

[19] H. C. Liu, M. Buchanan, and Z. R. Wasilewski, J. Appl. Phys. 68, 3780 (1990).

[20] N. Vodjdani, B. Vinter, V. Berger, E. Bockenhoff, and E. Costard, Appl. Phys. Lett., 59, 991).

[21] C. Liu, A. G. Steele, M. Buchanan, and Z. R. Wasilewski, J. Appl. Phys., 70, 7560 (1991).

[22] L. C. West and S. J. Eglash, Appl. Phys. Lett. 46, 1156 (1985).

[22] "Encyclopedia of Optical Engineering (volume 2)," edited by Ronald G Driggers, CRC Press, 2003, ISBN: 0824742516.

[23] Liou, K.N. (2002). An Introduction to Atmospheric Radiation, second edition, Academic Press, Elsevier, Amsterdam, 2002, ISBN 0124514510. Page 119.

[24] K. K. Choi, The Physics of Quantum Well Infrared Photodetectors (World Scientific, River Edge, New Jersey, 1997.

[25] R. H. Kingston, Detection of Optical and Infrared Radiation (Springer-Verlag, New York, (1978).

[26] F. F. Sizov, V. V. Vasiliev, J. V. Gumenjuk-Sichevska, Yu. E. Kamenev, S. A. Dvoretsky and N. N. Mikhailov, Proc. SPIE 7100, 71002C (2008).

[27] L. Esaki and L. L. Chang, Phys. Rev. Lett. 33, 495 (1974).

[28] R. Dingle, W. Wiegmann, and C. H. Henry, Phys. Rev. Lett. 33, 827(1974).

[29] A. G. U. Perera, W. Z. Shen, S. G. Matsik, H. C. Liu, M. Buchanan, and W. J. Schaff, Appl. Phys. Lett. 72, p1596,1998.

[30] S. data, "Quantum Phenomena in Nanostructure"

[31] S data, "Quantum Transport: from Atom to Transistor",ISBN: 9780521631457.

[32] C. J. Chen, K. K. Choi, L. Rokhinson, W. H. Chang, and D. C. Tsui, Appl. Phys. Lett. 75, $3210,1999$.

[33] M. O. Manasreh, F. Szmulowicz, D. W. Fischer, K. R. Evans, and C. E. Stutz, Appl.Phys. Lett. 57, 1790 (1990).

[34] K. K. Choi, C. J. Chen, and D. C. Tsui, J. Appl. Phys. 88, 1612, 2000.

[35] K. L. Tsai, K. H. Chang, C. P. Lee, K. F. Huang, J. S. Tsang, and H. R.Chen, Appl. Phys. Lett. 62, $3504,1993$.

[36] D. D. Coon and R. P. G. Karunasiri, Appl. Phys. Lett. 45, 649, 1984. 
[37] G. Hasnain, B. F. Levine, C. G. Bethea, R. A. Logan, J. Walker, and R. J. Mulik, Appl. Phys. Lett. 54, 2515, 1989.

[38] R. P. G. Karunasiri, J. S. Park, and K. L. Wang, Appl. Phys. Lett. 61, 2434, 1992.

[39] B. F. Levine, S. D. Gunapala, J. M. Kuo, S. S. Pei, and S. Hui, Appl. Phys.Lett. 59, 1864,1991.

[40] Semiconductor Quantum Wells and Superlattices for Long-Wavelength Infrared Detectors, edited by M. O. Manasreh, Artech House, Boston.1993.

[41] Semiconductor Quantum Wells and Superlattices for Long-Wavelength Infrared Detectors, edited by M. O. Manasreh Artech House, Boston, 1993.

[42] Levine B F 1993 J. Appl. Phys. 74 R1.

[43] Coon D D and Karunasiri G 1984 Appl. Phys. Lett. 45649.

[44] Schneider H, Walther M, Schonbein C, Rehm R, Fleissner J, Pletschen W, Braunstein J, Koidl P, Weimann G, Zeigler J, and Cabanski W., Physica E. 2000; 7: 101-107.

[45] Nash GR,Gordon NT, Hall DJ, Ashby MK, Little JC, Masterton G, Hails JE, Giess J, Haworth L, Emeny MT, Ashley T., Physica E 2004; 20: 540-5447.

[46] Liu H.C., Buchanan M, Wasilewski Z,R., Appl. Phys. Lett. 1998; 72:1682-1684.

[47] Liu H.C., Dudek R, Shen A, Dupont E, Song C.Y., Wasilewski ZR, Buchanan M, Appl. Phys. Lett. 2001; 79: 4237- 4239.

[48] L.-H. Peng and C. G. Fonstad, Appl. Phys. Lett. 62, 3342 (1993); L.-H. Peng, J. H. Smet, T. P. E. Broekaert, and C G. Fonstad, ibid. 62, 2413 (1993).

[49] X. Zhou, P. K. Bhattacharya, G. Hugo, S. C. Hong, and E. Gulari, Appl. Phys. Lett. 54, 855 (1989).

[50] Y. Shakuda and H. Katahama, Jpn. J. Appl. Phys. 29, L552 (1990).

[51] M. J. Kane, L. L. Taylor, N. Apsley, and S. J. Bass, Semicond. Sci. Technol. 3, 586 (1988).

[52] F. Miiller, V. Petrova-Koch, M. Zachau, F. Koch, D. Griitzmacher, R. Meyer,.H. Jiirgensen, and P. Balk, Semicond. Sci. Technol. 3, 797 (1988).

[53] H. Asai and Y. Kawamura, Appl. Phys. Lett. 56, 1149 (1990).

[54] H. Lobentanzer. W. KG\&. W. Stolz, K. Ploog, T. Elsaesser, and R. J. Biiuerle, Appl. Phys. LettP53, 571 (1988).

[55] H. Asai and Y. Kawamura, Appl. Phys. Lett. 56, 746 (1990).

[56] W. Trzeeiakowski and B. D. McCombe, Appl. Phys. Lett. 55, 891 (1989).

[57] A. Lorke, U. Merkt, F. Malcher, G. Weimann, and W. Schlapp, Phys. Rev. B 42, 1321 (1990).

[58] P. Helgesen, R. Sizmann, S. Lovold, and A. Paulsen, Proc. SPIE 1675 (1992).

[59] G. Temple, F. Miiller, N. Schwarz, F. Koch, G. Weimann, H. P. Zeindl, and I. Eisele, Surf. Sci. 228, 247 (1990).

[60] L.C. Chui, J.S. Smith, S. Margalit, A. Yariv, A Y Cho Infrared Phys A.Y. Cho, Infrared Phys. 23 93 (1983); J Vac Sci 23, 93 (1983); J. Vac. Sci.Technol. B 1, 376 (1983)

[61] J. H. Van de Merwe, J. Appl. Phys. 34, 123 (1962)

[62] R. People, and J. C. Bean, Appl. Phys. Let. 17 (3), 322 (1985).

[63] A. Haque, A. N. Khondker, J. Appl. Phys. 84, 10 (1998).

[64] W. Liu, D. H. Zhang, Z.M. Huang, and W. J. Fan, J.Appl.Phys., vol. 101, pp. 033114:1-7, 2007. 


\section{VITA}

Mehjabeen A Khan was born in Bangladesh in 1977. She received his B.Sc. and M.Sc. both in Electrical and electronic Engineering from Bangladesh University of Engineering and Technology (BUET)Bangladesh in 2001 and 2006 respectively. From January 2007, she has been pursuing her $\mathrm{PhD}$ in Electrical Engineering in the University of MissouriColumbia. From 2003-2005 she worked as a Lecturer at the BRAC University and then from 2005-2006 at United International University in Bangladesh. Her research area mainly focuses on the physics and design of quantum well infrared photodetectors. Her $\mathrm{PhD}$ dissertation research involves an investigation on asymmetric quantum well infrared photodetector structures formed through III-V semiconductor material system such as AlGaAs/ $\operatorname{In}_{x} \mathrm{Ga}_{(1-\mathrm{x})} \mathrm{As} / \mathrm{In}_{\mathrm{y}} \mathrm{Ga}_{(1-\mathrm{y})}$ As/AlGaAs. 